\title{
Ecology and shell chemistry of Loxoconcha matagordensis
}

\author{
T.M. Cronin ${ }^{\text {a,* }}$, T. Kamiya ${ }^{\text {b }}$, G.S. Dwyer ${ }^{\text {c }}$, H. Belkin ${ }^{\text {a }}$, C.D. Vann ${ }^{\text {a }}$, \\ S. Schwede ${ }^{\text {a }}$, R. Wagner ${ }^{\mathrm{a}}$ \\ ${ }^{\mathrm{a} 926 A}$ US Geological Survey, Reston VA, 20192, USA \\ ${ }^{\mathrm{b}}$ Department of Geological Sciences, Kanazawa University, Kanazawa, Japan \\ ${ }^{\mathrm{c}}$ Nicholas School of the Environment, Earth and Ocean Sciences, Duke University, Durham, NC, 27708, USA
}

Received 31 August 2001; received in revised form 31 August 2001; accepted 25 May 2005

\begin{abstract}
Studies of the seasonal ecology and shell chemistry of the ostracode Loxoconcha matagordensis and related species of Loxoconcha from regions off eastern North America reveal that shell size and trace elemental $(\mathrm{Mg} / \mathrm{Ca}$ ratio) composition are useful in paleothermometry using fossil populations. Seasonal sampling of populations from Chesapeake Bay, augmented by samples from Florida Bay, indicate that shell size is inversely proportional to water temperature and that $\mathrm{Mg} / \mathrm{Ca}$ ratios are positively correlated with the water temperature in which the adult carapace was secreted. Microprobe analyses of sectioned valves reveal intra-shell variability in $\mathrm{Mg} / \mathrm{Ca}$ ratios but this does not strongly influence the utility of whole shell $\mathrm{Mg} / \mathrm{Ca}$ analyses for paleoclimate application.
\end{abstract}

Published by Elsevier B.V.

Keywords: Ostracoda; Shell chemistry; Ecology

\section{Introduction}

The application of ostracode shell trace element chemistry to paleoenvironmental reconstruction requires an understanding of processes that influence the chemical composition of the calcitic shell. In the case of magnesium uptake into the shell (measured by $\mathrm{Mg} / \mathrm{Ca}$ ratios), the most important factors include water temperature (Cadot and Kaesler, 1977; Chivas et al., 1986; Corrège, 1993; Dwyer et al., 1995; Corrège and De Deckker, 1997), salinity and water chem-

\footnotetext{
* Corresponding author.

E-mail address: tcronin@usgs.gov (T.M. Cronin).
}

istry (Engstrom and Nelson, 1991; Wansard et al., 1998; Dwyer and Cronin, 2001; Holmes and Chivas, 2002) and ostracode metabolism (Chivas et al., 1983; De Deckker et al., 1999). Equally important for application to paleoclimate reconstruction is knowledge of a species' seasonal population ecology because this will determine under what conditions the species secretes its adult shell. If both the processes and environmental factors controlling the uptake of magnesium and the timing of adult molting are known, the ostracode can provide a powerful tool for the study of interannual and decadal-scale climate variability.

The genus Loxoconcha is potentially an ideal ostracode for paleoenvironmental reconstruction using ecol- 
ogy and shell chemistry in middle and low latitude coastal environments. Loxoconcha first appeared in the Paleogene, is common in Cenozoic fossil assemblages, and today has a cosmopolitan distribution. Kempf (1986) estimates that the genus is represented by more than 150 living species and 350 fossil species. Thus, the development of a quantitative temperature-magnesium/ calcium relationship for Loxoconcha would be important for shallow-water Cenozoic paleoceanography.

The current paper is an integrated study of the ecology, morphology, and shell chemistry of the epiphytal species Loxoconcha matagordensis (Swain, 1955) from Chesapeake Bay (Fig. 1). Loxoconcha matagordensis is a dominant species in shallow coastal habitats along the North American Atlantic and Gulf coasts where it lives primarily on blades of the seagrass Zostera marina at salinities ranging from $\sim 15$ to $30 \mathrm{ppt}$. This species was first reported from Chesapeake Bay by Tressler and Smith (1948) as Loxoconcha impressa (Baird), and formally described by Swain (1955) from Texas bays. It has since been the subject of several ecological studies (e.g., Morales, 1966; King and Kornicker, 1970).

In the first part of this paper, we present ecological (population structure, density) and morphological (carapace length) data from monthly and bimonthly sampling during 1999-2000 from Guinea Marsh and Goodwin Island at the mouth of the York River, a tributary entering southern Chesapeake Bay, Virginia (Fig. 2). Supplementary material from Florida Bay was also used to examine the carapace length-temperature relationship in Loxoconcha matagordensis. In the second part, we present evidence for temperature con-

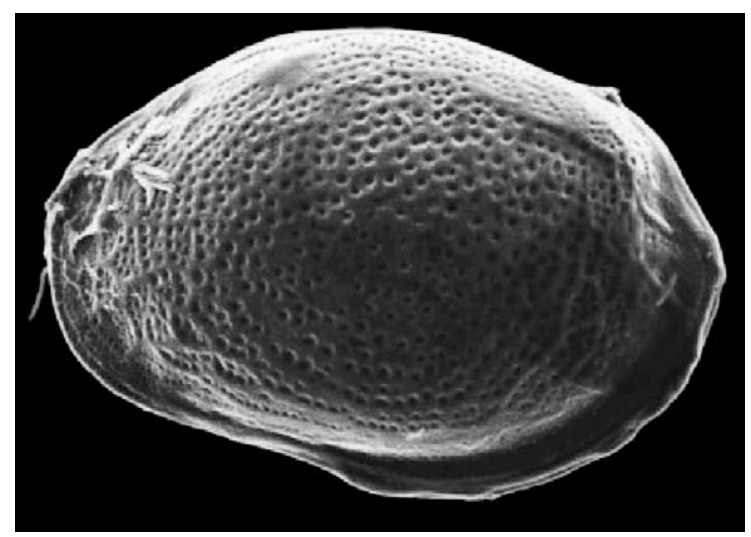

Fig. 1. Scanning electron photomicrograph of female left valve of Loxoconcha matagordensis. Shell is about $600 \mu \mathrm{m}$ in length. trol of magnesium/calcium ratios obtained by direct current plasma (DCP) emission spectrometry for adult Loxoconcha matagordensis from the York River samples. In addition to Loxoconcha matagordensis, measurements were also made on Loxoconcha sp. from the main channel of Chesapeake Bay and the continental shelf off the bay's mouth, and Loxoconcha impressa from the continental shelf. We also present evidence for intra-shell variability in $\mathrm{Mg} / \mathrm{Ca}$ ratios obtained by electron probe microanalyzer (microprobe). In a companion paper the ecological and $\mathrm{Mg}$ / $\mathrm{Ca}$ shell calibration presented here are applied to the reconstruction of 2200 year temperature record of Chesapeake Bay (Cronin et al., 2003).

\section{Materials and methods}

Guinea Marsh and Goodwin Island are located at the mouth of the York River, a tributary to the large partially mixed temperate estuary Chesapeake Bay. The two sites are characterized by healthy beds of the seagrass Zostera marina which have been monitored for seagrass and water quality by scientists at the Virginia Institute of Marine Sciences (VIMS) for more than a decade (Moore and Berry-Niekirk, personal communication). Thus, they provide an ideal natural laboratory to examine seasonal variability in ecology, morphology, and shell chemistry of Loxoconcha matagordensis.

Sampling of Zostera beds living in $\sim 1 \mathrm{~m}$ water depth at Guinea Marsh and Goodwin Island (Fig. 2) was carried out from February 1999 to December 2000 by the U.S. Geological Survey (USGS) in cooperation with VIMS. Zostera samples were taken monthly during fall and winter and bimonthly during spring and summer. During 1999, Zostera was collected either by hand or from material brought up on the ship's anchor. Because some samples did not yield abundant ostracode specimens, a different method was used during 2000, whereby Zostera was collected using a post-hole device lowered into the water to scoop up sediment and growing seagrass. After collection, Zostera blades were quickly separated from bottom sediment so that epiphytal species could be separated from those living on the bottom. Separate Zostera and sediment samples were then shipped overnight in plastic sample bags sealed in cooled containers to the USGS ostracode lab in Reston, VA. Water salinity and temperature were taken at the time of sampling. It is noteworthy that the 2-year 


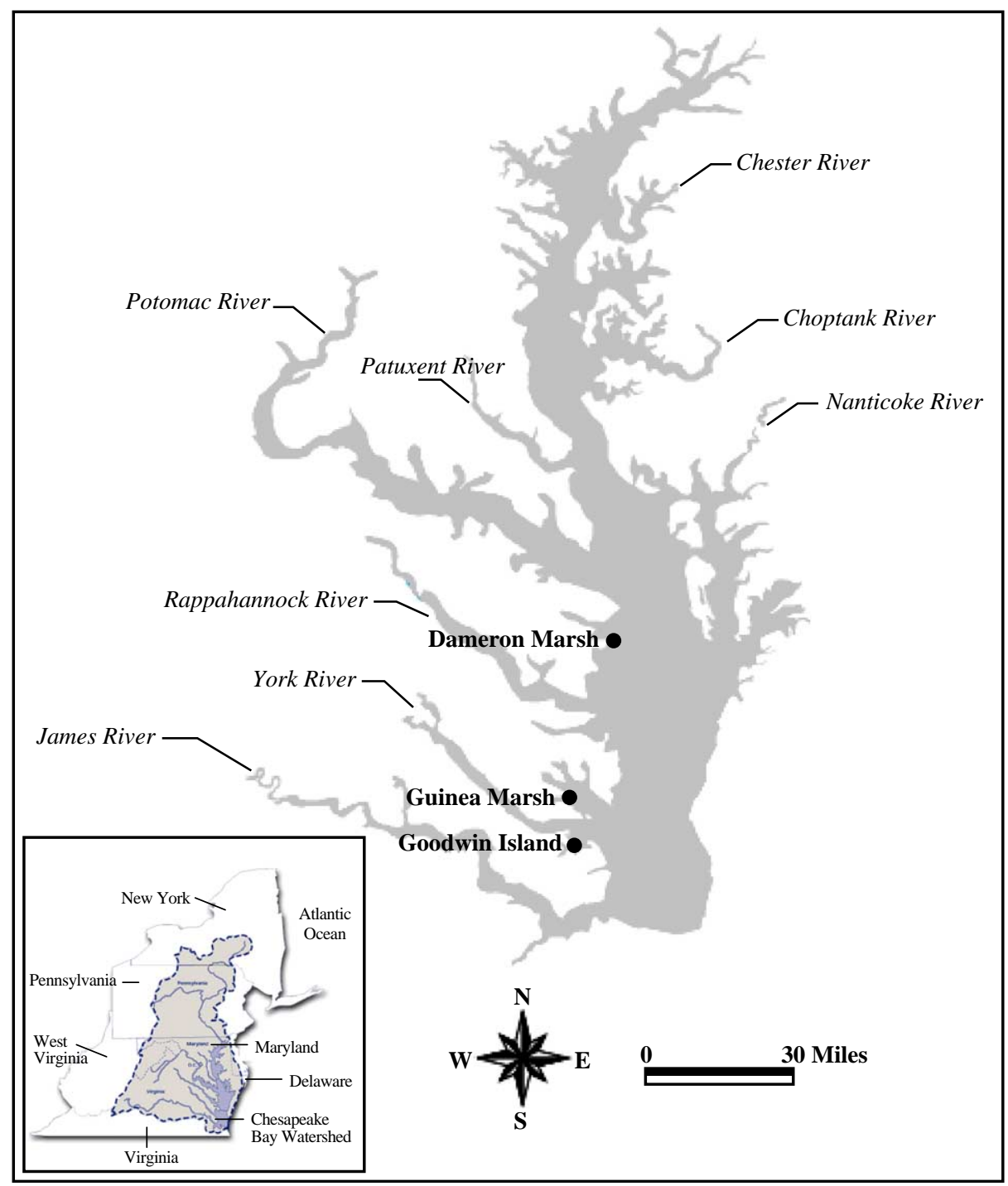

Fig. 2. Map showing location of York River and Guinea Marsh and Goodwin Island sampling sites. Also shown are Patuxent and Rappahannock River tributaries of Chesapeake Bay where early studies of ostracodes discovered Loxoconcha matagordensis living on Zostera.

temperature and salinity curves obtained in our study are within $1{ }^{\circ} \mathrm{C}$ and $1-2 \mathrm{ppt}$ of temperature and salinity values from the main stem of the bay obtained from the Chesapeake Bay Program for the same period.

To isolate Loxoconcha from Zostera, the blades of seagrass were washed through a $63-\mu \mathrm{m}$ sieve at the USGS in Reston. Loxoconcha specimens were picked using a fine brush under a light microscope and placed on microfaunal slides. Zostera from each collection dates were weighed to obtain estimates of the relative density of ostracodes for each sample.
In contrast to Chesapeake Bay, Florida Bay is a shallow, subtropical carbonate embayment subjected to wide spatial and temporal salinity extremes. Loxoconcha matagordensis also thrives in Florida Bay where it lives mainly on seagrass, most commonly on Thalassia testudinum (Cronin et al., 2001). Seagrass samples in Florida Bay were taken by hand in February and July 1998-2000 by Dr. L. Wingard (USGS) as part of a larger program investigating the paleoecology of Florida Bay. Samples were washed and picked in the same manner as those from Chesapeake Bay. Cronin et 
al. (2001) gave location, salinity and temperature data for the ostracode samples used in the current study.

The ostracode population and their shell length data from Guinea Marsh and Goodwin Island are given in Appendix A; data from Florida Bay in Appendix B.

Geochemical analyses were carried out on adult individuals of three species of Loxoconcha. Most of the specimens were taken from the 1999 samples from Guinea Marsh and Goodwin Island, York River. Additional Loxoconcha matagordensis were obtained from Dameron Marsh, Chesapeake Bay collected March, 1999, and from Bogue Sound, North Carolina collected August 1999. Also included are specimens of Loxoconcha sp. collected from the main channel of Chesapeake Bay in June and September, 1999, on the $R / V$ Orion and Loxoconcha impressa collected from Atlantic shelf and slope surface sediments during a large study in the 1960s (Hazel, 1970, Valentine, 1971).

Individual specimens were soaked overnight in Clorox bleach to remove organic material which can cause anomalously high $\mathrm{Mg} / \mathrm{Ca}$ ratios in ostracodes (Kondo et al., 2005). They were then washed and sonicated in two-step bath of deionized water, then dissolved in 4 to $30 \mathrm{ml}$ of $0.05 \mathrm{~N}$ nitric acid, depending on valve weight. Between 1 and 14 (mean of 6) individuals per sample were analyzed for magnesium and calcium (strontium and sodium also was measured but these are not considered in this paper) at Duke University on a Spectrascan 7 direct current plasma (DCP) emission spectrometer following the procedures in Dwyer et al. (1995). The analytical precision was $\pm 3 \%$ based on replicate analyses of samples and standards. An in-house limestone standard Duke PE3 yielded $\mathrm{Mg} / \mathrm{Ca}$ of $8.50 \mathrm{mmol} / \mathrm{mol} \pm 0.04(1 \sigma)(n=40)$.

Several specimens were also analyzed on a JEOL JXA-8900R Electron Probe Microanalyzer (microprobe) in order to evaluate intra-shell $\mathrm{Mg} / \mathrm{Ca}$ variability. Specimens were mounted lateral (convex) side up on glass slides in Buehler ${ }^{\circledR}$ Epoxide Resin and Hardener, ground with a 5- $\mu \mathrm{m}$ grit and polished with $1-\mu \mathrm{m}$ polish paper. This procedure resulted in a ring-like cross-section through the carapace nearly perpendicular to the shell surface. Petrographic examination assured that the sectioned shell surface was smooth enough for microprobe analysis. Point measurements were made at several locations in the central part of three shells and a continuous sweep of the shell was also made.

\section{Results}

\subsection{Population density and age structure}

Loxoconcha matagordensis exhibits strong seasonal ecological patterns in terms of its density on Zostera blades in Chesapeake Bay and its tributaries. In early ecological studies of ostracodes from the Patuxent River (Fig. 2), Tressler and Smith (1948) found this species (referred to as Loxoconcha impressa) was almost absent during winter months in 1941 and 1942 but its abundance rose sharply in May and was maintained through October. Elliott et al. (1966) also found that Loxoconcha matagordensis lived almost exclusively on Zostera in the Rappahannock River. Additionally, Grossman (1965) and Morales (1966) also found it was the dominant species in Redfish Bay, Texas and Laguna de Terminos, Mexico in the Gulf of Mexico. King and Kornicker (1970) carried out seasonal sampling of ostracodes in Texas bays in 19581959 and observed that Loxoconcha matagordensis was rare $\left(<50\right.$ individuals per $\left.\mathrm{m}^{2}\right)$ during winter months, increased during spring ( 200 individuals per $\left.\mathrm{m}^{2}\right)$ and rose sharply to peak abundance in July $\left(\sim 600\right.$ per $\left.\mathrm{m}^{2}\right)$ (Fig. 3). The seasonal increases in abundance coincided with a rise in water temperature from 15 to $>25{ }^{\circ} \mathrm{C}$.

Our results on the density of Loxoconcha matagordensis, measured as the number of adults per 100 g of Zostera, from Chesapeake Bay (Fig. 4) confirm those of both Tressler and Smith (1948) and King and Kornicker (1970). There is a modest increase in the number of adult Loxoconcha matagordensis during spring and a sharper rise during summer of the year 2000 at both Guinea Marsh and Goodwin Island sites.

In addition to seasonal changes in the density of adult specimens, there are important changes in the proportions of adult and juvenile instars in Chesapeake Bay populations (Fig. 5). Despite the different sampling methods used during 1999 and 2000, there were a relatively large proportion of juveniles during the late summer and fall of both years and relatively few during the winter months. This relationship is evident at both Guinea Marsh and Goodwin Island. The abundance of juveniles in summer represents offspring produced during seasonal breeding beginning in spring and persisting into the early Fall. It is possible that the drop in juveniles during August 1999 (Fig. 5) repre- 


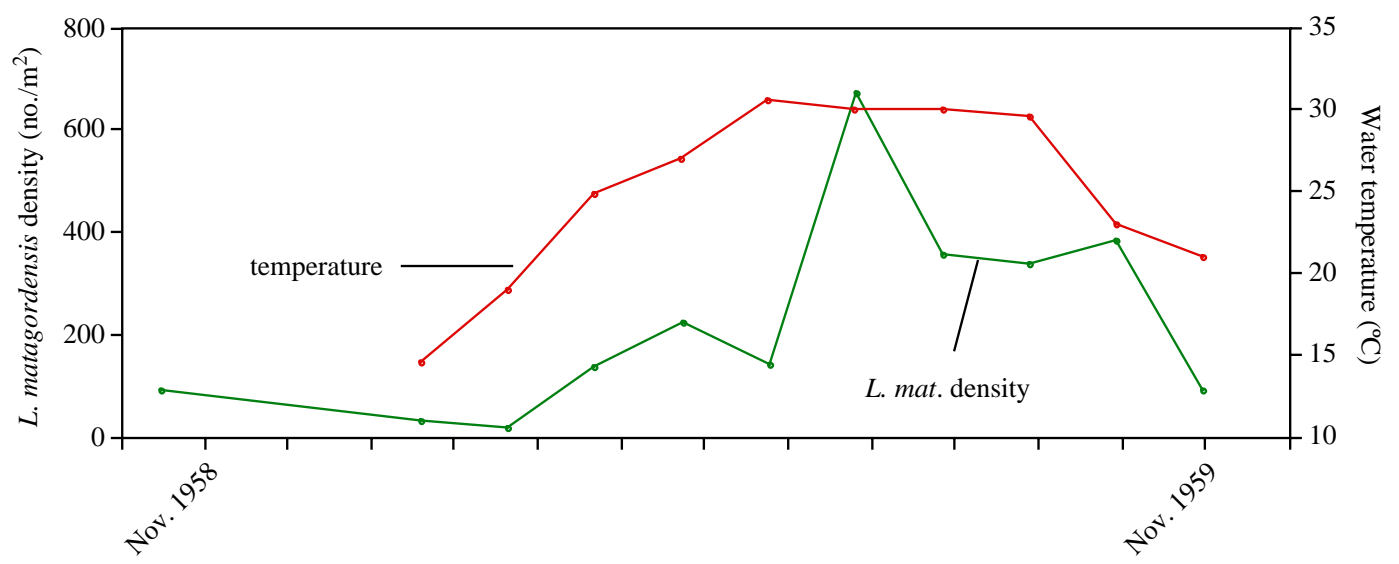

Fig. 3. Plot of density of Loxoconcha matagordensis and water temperature in Redfish Bay, Texas for the period November 1958 until November 1959 from King and Kornicker (1970).

sents a brief period in between separate spring and late summer breeding seasons, a concept supported by the trends in carapace length described below. It should also be emphasized that many factors might have influenced the relative proportions of adults and juve- niles obtained in any particular sample, including artifacts of weather conditions and water quality. Still, the broad patterns identified at both sites are consistent with our understanding of the ecology of Loxoconcha species living in temperate regions, where there are
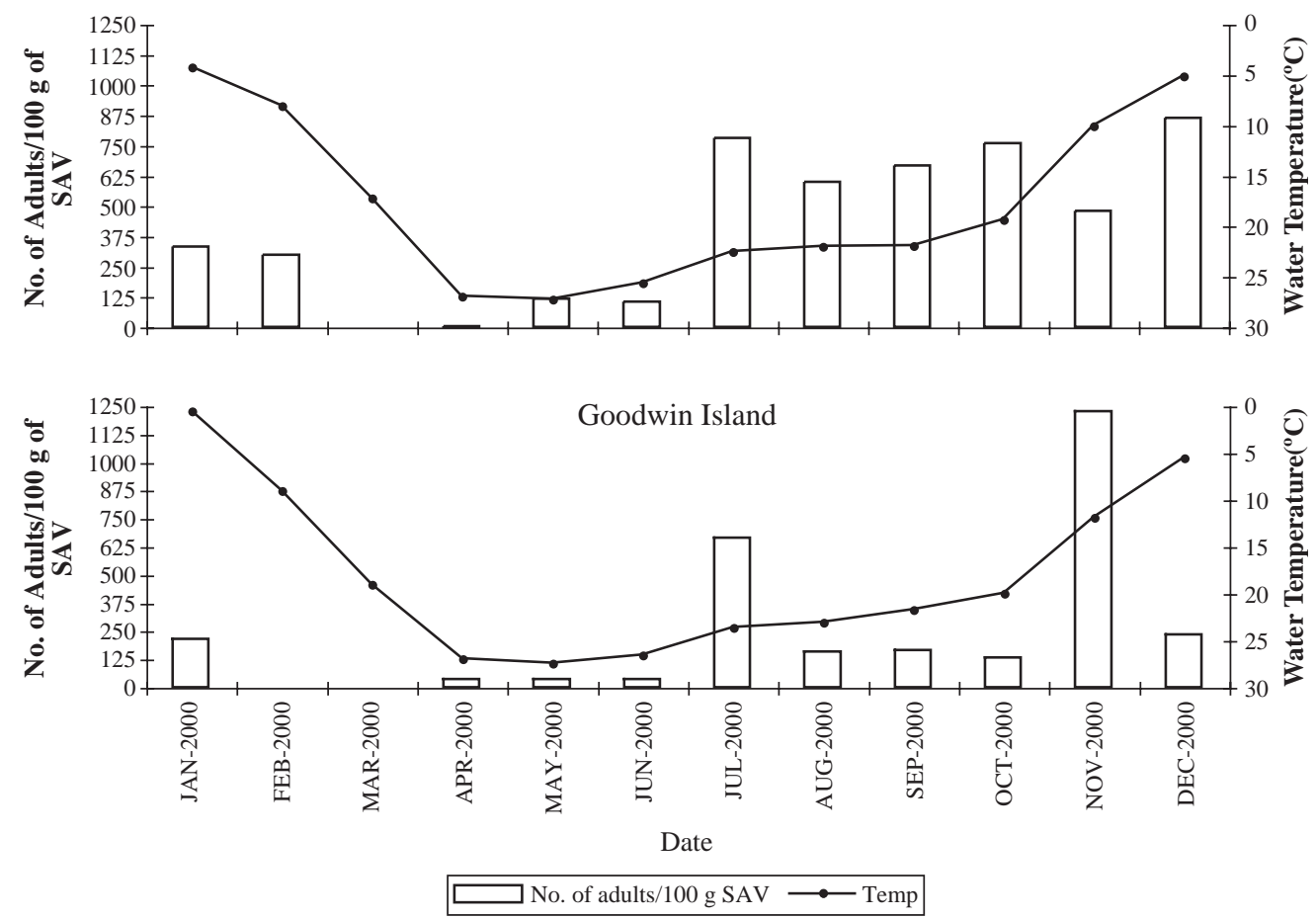

Fig. 4. Seasonal variability of abundance of adult Loxoconcha matagordensis (females and males) at Guinea Marsh and Goodwin Island, York River mouth, Chesapeake Bay during the year 2000, measured as density in subaquatic vegetation (SAV). Quantities of adult Loxoconcha matagordensis are generally lowest between February and June at both sites, and rise during summer and early fall. 


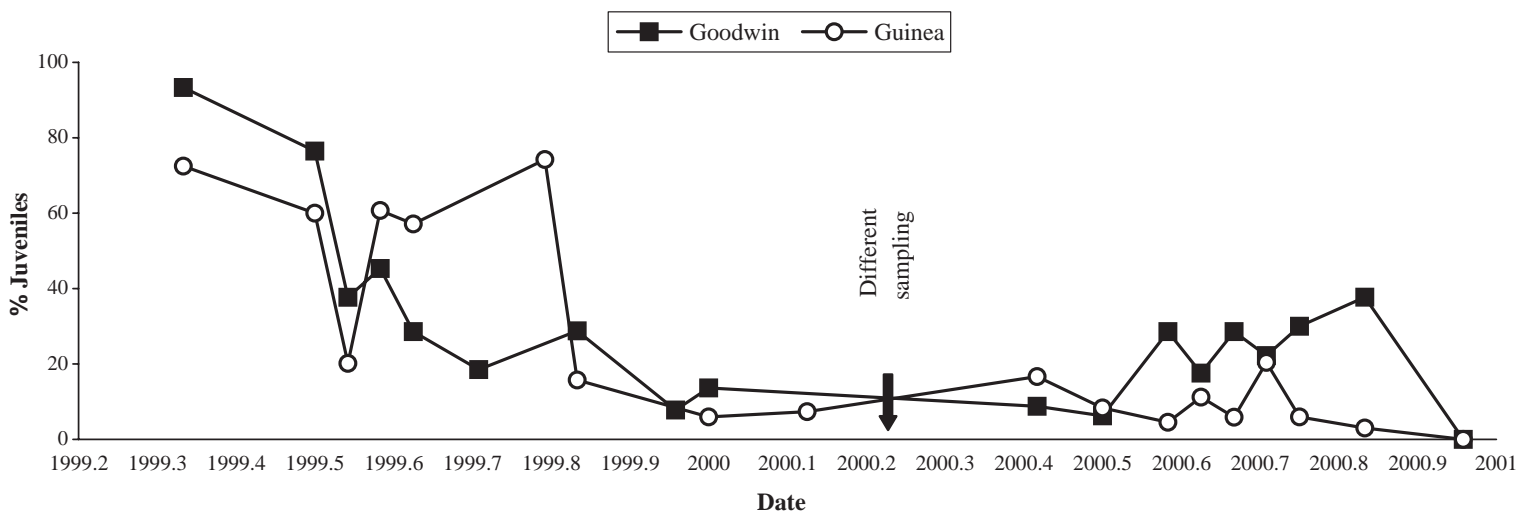

Fig. 5. Seasonal variability of juvenile Loxoconcha matagordensis (total of A-1 to A-8 instars) for the period 1999-2000 at Guinea Marsh and Goodwin Island sites, York River mouth, Chesapeake Bay, Virginia. Sampling method changed in April, 2000 (see Materials and methods).

large seasonal temperature oscillations, and the relationship of this epiphyte to the seasonal ecology of its host plant Zostera.

\subsection{Trends in shell preservation}

Kamiya (1988) demonstrated that the state of shell preservation (clean and translucent to extremely dirty and brown, referred to by Kamiya as "fouled") in living specimens of Loxoconcha japonica from Aburatsubo Cove, Japan is related to the age of the adult ostracode. Clean and translucent shells of Loxoconcha japonica represent adults that were the most recently secreted. With age (weeks to months), adults become progressively dirtier because epidermal cells and the epicutical become colored and/or organic detritus adheres to the outside of the shell. The level of organic detritus in the water is therefore an important factor in how quickly an adult carapace can lose its original translucent appearance; the more organic material the more quickly an adult can become discolored.

Kamiya (1988) also discovered an inverse relationship between carapace length and water temperature in Loxoconcha japonica. He showed that by separating adults into three categories (which we refer to as clean, intermediate, and dirty), and by measuring adult carapace size, he could recognize seasonal patterns in Loxoconcha japonica populations related to the age of the individuals and the timing of breeding and production of young instars. He recognized that the larger and dirtier adults were found in populations collected during the cooler seasons and surmised that these individuals had wintered over from the previous fall.
Conversely, smaller, translucent (recently secreted) adults occurred in late spring and summer, peaking in abundance in July.

We investigated the relationship between carapace length and water temperature in Loxoconcha matagordensis using carapace length measurements in males and females from Guinea Marsh and Goodwin Island for the 2-year collection period. A total of 936 females and 688 males were measured under a light microscope for this analysis. Our results reveal a seasonal size pattern in Loxoconcha matagordensis similar to that observed in Loxoconcha japonica. Fig. 6 shows that during the winter and spring of 1999 very few clean shells were found because those collected during this time represented relatively old adult individuals. As is the case with Loxoconcha japonica, July populations of Loxoconcha matagordensis adults are both clean and relatively small (length $\sim 600-605 \mu \mathrm{m}$ ) and appear to represent individuals from the spring breeding season. In sum, despite limited numbers of specimens of Loxoconcha matagordensis from several samples, the similarity in terms of population structure (age inferred from carapace preservation) for both species indicate that Kamiya's method of age identification in Loxoconcha japonica appears to apply to Loxoconcha matagordensis as well.

\subsection{Seasonal and geographic variability in carapace length}

To further examine the inverse relationship between size and temperature in Loxoconcha, we plotted mean female and male carapace length for 


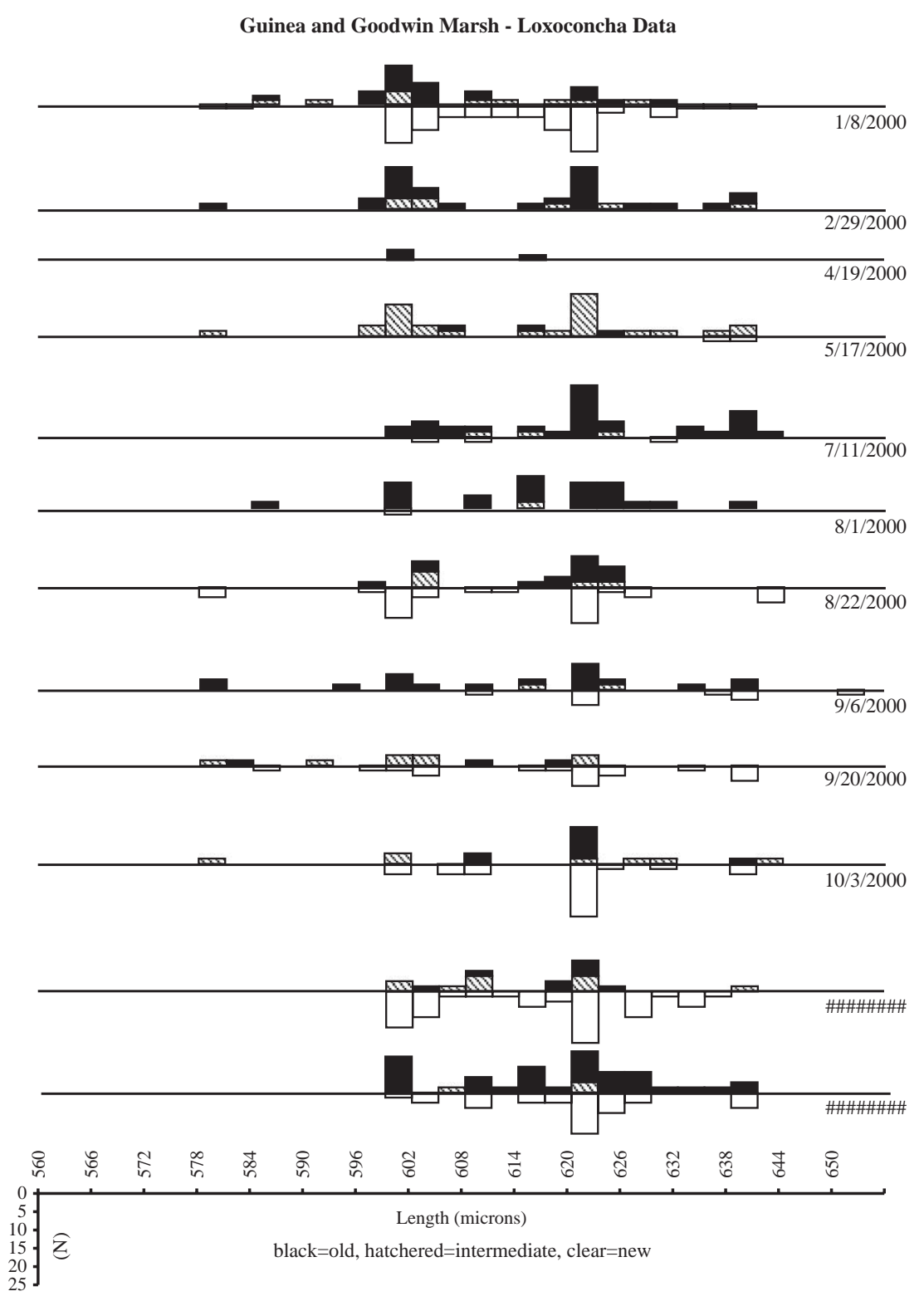

Fig. 6. Age distribution and carapace length of adult female Loxoconcha matagordensis in York River. Bars represent data pooled from Guinea Marsh and Goodwin Island with different colored bars indicating the approximate age of the carapace; clear=new carapace, hatchered=intermediate carapace, black=old carapace. The rate of carapace discoloration is generally more rapid in summer months due to an increased quantity of floating detritus in the water.

combined Guinea Marsh and Goodwin Island populations against measured water temperature $\left(T_{\mathrm{m}}\right)$ for the entire 1999-2000 study period (Fig. 7). Several important points emerge from this figure. First, there is a clear parallel between male (size range $680-715$ $\mu \mathrm{m})$ and female $(590-640 \mu \mathrm{m})$ length variability dur- ing different seasons. Both sexes show a smaller annual range of lengths during the year 2000 compared to 1999. The reason for this greater variability during 1999 is unclear although it is unlikely to be related to temperature as seasonal patterns were similar in both years. 

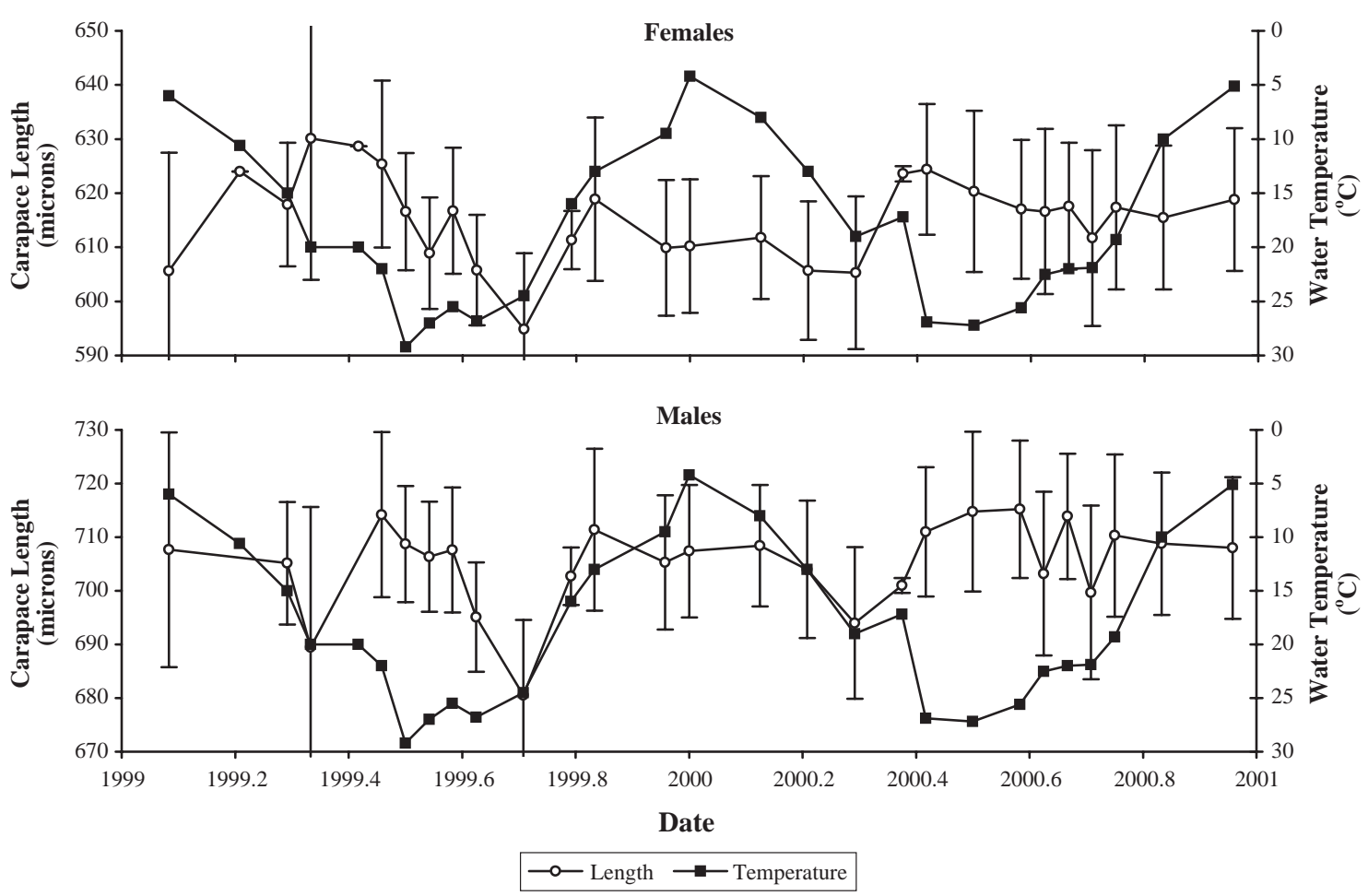

Fig. 7. Relationship between carapace length of female and male Loxoconcha matagordensis from York River and water temperature for the two year period 1999-2000. Carapace length and water temperature data are pooled from two sites, Guinea Marsh and Goodwin Island at the mouth of the York River. Graph shows mean carapace lengths and vertical bars represent \pm 1 standard deviation.

Second, there appears to be a 1-2 month "lag" between the spring-summer increase in $T_{\mathrm{m}}$ from 15 to $30{ }^{\circ} \mathrm{C}$ and the June through September period when there is a significant decrease in shell size. The most likely explanation for this trend is that many or most shells collected during these months actually secreted their adult shell as much as 1-2 months before the collection date. In other words, secretion water temperature $\left(T_{\mathrm{S}}\right)$ is not equivalent to measured water temperature $\left(T_{\mathrm{m}}\right)$. Although the exact age, and therefore the timing of secretion cannot be determined, and these collections most likely include adults that grew at several periods during the spring breeding season, the fact that some adults from this period have brown coloration suggests that many are 1-2 months old.

In contrast to the spring-summer trend in decreasing length, the increase in carapace length in both males and females during fall (September-November) tracks closely to the seasonal drop in $T_{\mathrm{m}}$ (Fig. 7). This trend is more apparent for the year 1999. It appears that the adults collected during fall months had secreted their adult shell very close to the collection date; that is, there is no "lag" time between secretion and collection and $T_{\mathrm{s}}$ approximates $T_{\mathrm{m}}$.

If a length-temperature relationship is a characteristic feature of Loxoconcha matagordensis, then it should also be evident in populations from other regions. Consequently, we compared size in female adult length for Loxoconcha matagordensis from cool and warm seasons in Chesapeake populations with populations collected from Florida Bay in February and July, 1998 (Fig. 8). Florida Bay is located in a subtropical region with a smaller seasonal temperature range $\left(\sim 12\right.$ to $\left.>30^{\circ} \mathrm{C}\right)$ than Chesapeake Bay $(\sim 0$ to 29 ${ }^{\circ} \mathrm{C}$ ) and thus its ostracode population provides a warm water end-member for the species.

Fig. 8 shows greater size in northerly populations (Chesapeake Bay) and those that grew during the cold seasons. While we cannot be certain exactly when the Florida adults secreted their shells (i.e., if $T_{\mathrm{m}}$, equals $T_{\mathrm{s}}$ ), we assume these February and July populations are representative of cool and warm season size 

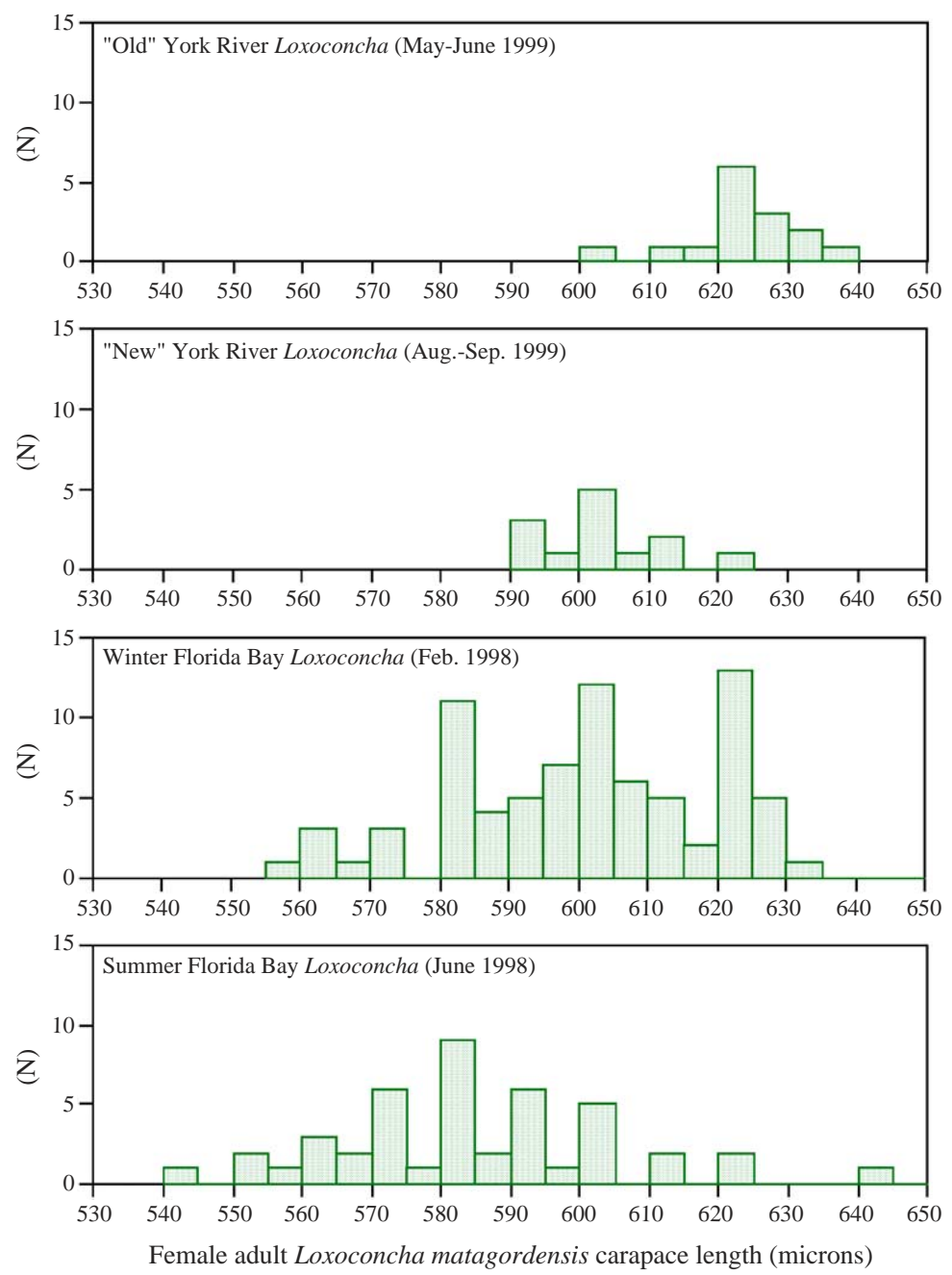

Fig. 8. Histogram showing size distribution of adult female Loxoconcha matagordensis that grew during winter and summer months in Chesapeake and Florida Bays. Adults that grew in warmer water (summer season, lower latitude) are generally smaller than those grown in cooler water (winter season, higher latitude).

classes, respectively. Nonetheless, Figs. 7 and 8 generally provide strong evidence that temperature variability resulting from both seasonality and geographic location result in size difference in populations of this species.

\subsection{Carapace size-temperature regression model}

Because carapace size might be a potential paleotemperature indicator, we derived several provisional regression models to express the inverse size-temperature relationship in Loxoconcha matagordensis. We first considered whether the preservation of the shell is a factor, that is, if we could determine whether $T_{\mathrm{m}}$ equals $T_{\mathrm{s}}$. Fig. 9A shows an inverse relationship between mean female length and $T_{\mathrm{m}}$ using clean, intermediate, and dirty adult females from Guinea Marsh, Goodwin Island and two samples collected from Florida Bay during February and July, 1998-2000. Fig. 9B includes only clean and intermediate preservation female adults from March to November 1999 (Guinea and Goodwin samples) and the two Florida Bay populations. Although both plots reveal an inverse relationship between length and temperatures, there is little differ- 
A

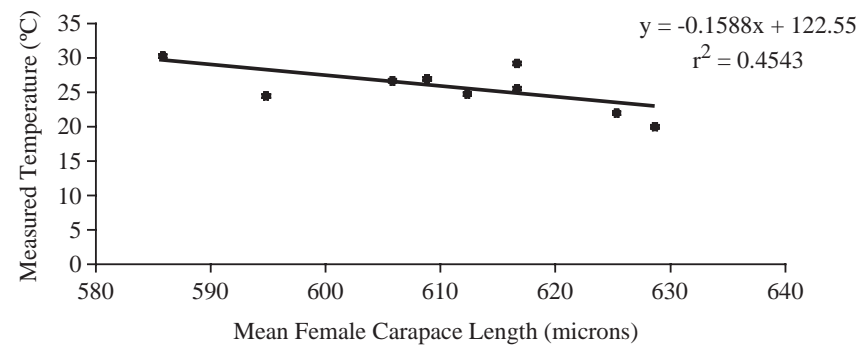

B

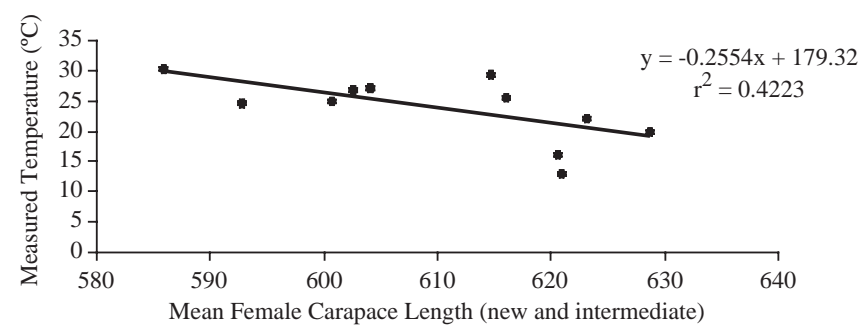

$\mathrm{C}$

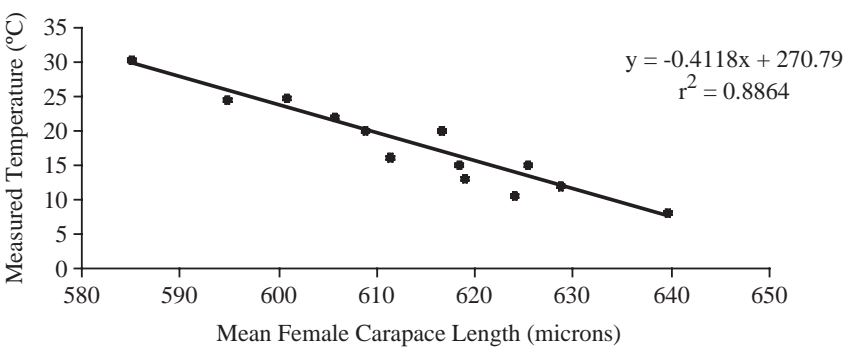

D

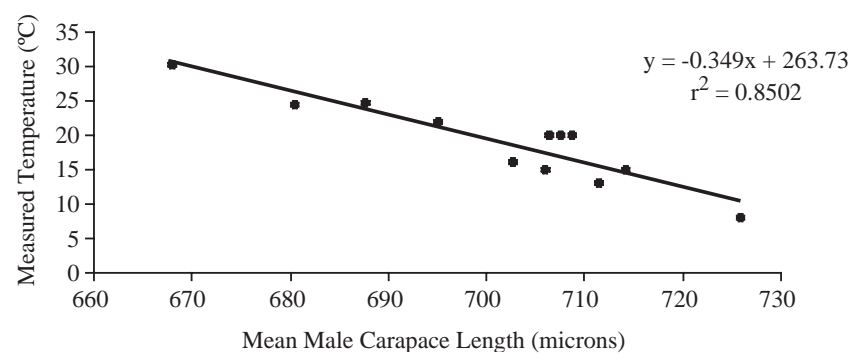

Fig. 9. Linear regressions of carapace length of adult Loxoconcha matagordensis and water temperature. (A) Mean female values using pooled populations from Guinea Marsh and Goodwin Island, Chesapeake Bay (June-September, 1999) and mean female values using pooled populations from Florida Bay (152 total females) from February to July, 1998. Includes clean, intermediate and dirty shells and $T_{\mathrm{m}}$ (measured) for temperature values. (B) Same as in panel A except Chesapeake values from June to November and only clean and intermediate shells included. (C) Same as panel B except $T_{\mathrm{s}}$ (estimated secretion temperature) used for June-September months. (D) Same as in panel C expect for males.

ence between the two and the correlation coefficients in both regressions are similar $\left(r^{2}=0.42-0.45\right)$. The scatter is probably due to the fact that individuals comprising each sample population secreted their adult carapaces at slightly different times prior to the date of collection, and thus at slightly different water temperature.
We attempted to use a more realistic estimate of $T_{\mathrm{m}}$ on the basis of the seasonal population ecology in Loxoconcha matagordensis. In Fig. 9C and D, the same Chesapeake and Florida Bay data are plotted for both females and males, except for the June-September data points, where we used temperature taken 2 
months prior to collection time because of the apparent lag time between collection and adult secretion. We used the actual measured temperatures at collection time for September-November, under the assumption the $T_{\mathrm{m}}$ is equivalent to $T_{\mathrm{s}}$ for this period. A single winter sample from $T_{\mathrm{m}}=6{ }^{\circ} \mathrm{C}$ was omitted because it is unlikely that adults from Chesapeake populations grew at such low temperatures. These plots reveal that both females and males exhibit similar inverse relationship between length and temperature and that both have a much improved correlation $\left(r^{2}=0.85\right.$ and 0.88 ). Although these procedures involved a degree of subjectivity and further work is needed, we believe the regression model illustrated in Fig. 9C and D best captures the carapace size-temperature relationship in Loxoconcha matagordensis based on its population ecology, zoogeography and our experience with other species of Loxoconcha.

\subsection{Seasonal variability in magnesium/calcium ratios of Loxoconcha matagordensis in Chesapeake Bay}

In this part of the paper we examine the relationship between $\mathrm{Mg} / \mathrm{Ca}$ ratios and water temperature in populations of Loxoconcha matagordensis collected in February through December, 1999 mainly from Guinea Marsh (a few measurements were made on individuals from Goodwin Island, Fig. 10). Mean $\mathrm{Mg} / \mathrm{Ca}$ ratios pooled from the two sites were about $25 \mathrm{mmol} / \mathrm{mol}$ in
February-April, rose to maximum levels of $45-48$ $\mathrm{mmol} / \mathrm{mol}$ in July and August, and fell again to $\sim 25$ $\mathrm{mmol} / \mathrm{mol}$ in December. The results suggest a positive relationship between mean $\mathrm{Mg} / \mathrm{Ca}$ ratio and seasonal changes in $T_{\mathrm{m}}$ for much of the year. However, it should be kept in mind in light of the prior discussion, that the values for $T_{\mathrm{m}}$, may slightly overestimate $T_{\mathrm{s}}$ for an unknown number of individuals which secreted their adults shells 1-2 months prior to collection date.

Before proceeding, it should be noted that $\mathrm{Mg} / \mathrm{Ca}$ ratios in Chesapeake Bay water are relatively constant across the annual salinity range at the Guinea and Goodwin sites ( 18-23 ppt for 1999) and thus salinity variability is unlikely to be an important factor in explaining the observed pattern in Loxoconcha $\mathrm{Mg} / \mathrm{Ca}$ ratios. This is in contrast to Florida Bay waters where salinity (0$40 \mathrm{ppt})$ and $\mathrm{Mg} / \mathrm{Ca}$ molar ratios $(0-5 \mathrm{mmol} / \mathrm{mol})$ are positively correlated due to fresh/marine water mixing processes (Dwyer and Cronin, 2001).

\subsection{Magnesium/calcium-temperature calibration equation}

To further examine the Loxoconcha $\mathrm{Mg} / \mathrm{Ca}$-temperature relationship, we assembled a group of samples from other regions along the eastern United States representing what we consider the entire temperature range at which adult Loxoconcha are secreted. In addition to the Guinea Marsh and Goodwin Island samples,

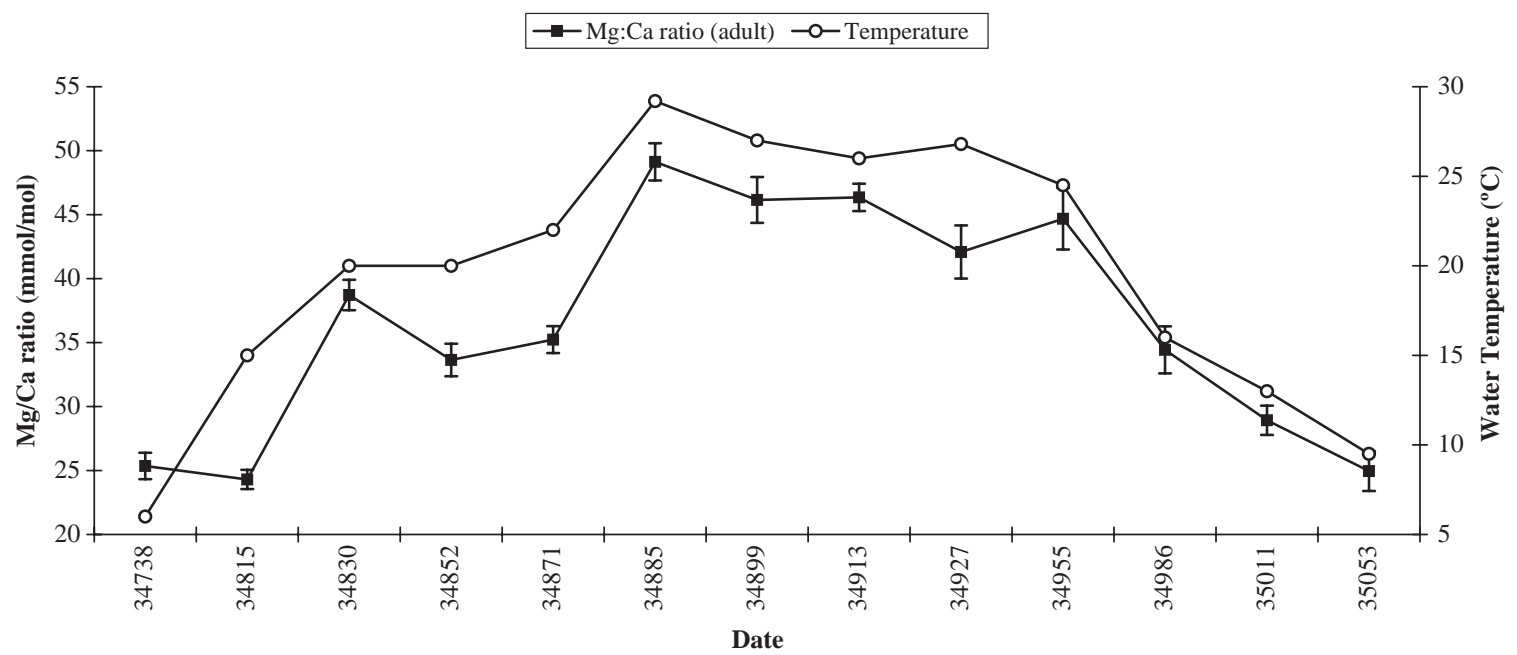

Fig. 10. Relationship between mean $\mathrm{Mg} / \mathrm{Ca}$ ratio of adult Loxoconcha matagordensis from Guinea Marsh and Goodwin Island (pooled for each sampling date), Chesapeake Bay and $T_{\mathrm{m}}$ for the year $1999 . \mathrm{Mg} / \mathrm{Ca}$ ratios are in $\mathrm{mmol} / \mathrm{mol}$, vertical bars are 1 standard error. 
we measured $\mathrm{Mg} / \mathrm{Ca}$ ratios in Loxoconcha matagordensis from Bogue Sound, North Carolina collected in August 1999 and from Dameron Marsh, Chesapeake Bay collected in March 1999, Loxoconcha sp. collected from the channel of Chesapeake Bay in June and September 1999, and Loxoconcha sp. and Loxoconcha granulata collected from surface sediments on the continental shelf of eastern North America. A total of 156 individual adult specimens from 36 samples were used in this analysis (Appendix C).

Fig. 11 shows the resulting regression model constructed from 36 mean $\mathrm{Mg} / \mathrm{Ca}$ values covering a range in $T_{\mathrm{m}}$ of $7{ }^{\circ} \mathrm{C}$ to $30^{\circ} \mathrm{C}$. The correlation coefficient for this model is $r^{2}=0.82$ and the standard error is $2.6{ }^{\circ} \mathrm{C}$. Other regression equations using slight modifications of this data set (i.e., inclusion of A-1 instars and pooling all samples from the June and September 1999 Chesapeake Bay collections) did not produce any major differences in slope, correlation coefficient, or standard error.
It is important to point out several limitations of these data. First, the inclusion of three species introduces potential interspecific taxonomic variability in $\mathrm{Mg} / \mathrm{Ca}$ ratios. This does not appear to be of major concern for several reasons. These species are closely related to one another and their stratigraphic distribution suggests they evolved during the Pliocene and are all adapted to generally similar temperate climate regimes. This suggests similar ecological requirements, and we assume this also means they all have similar physiology with respect to the molting process. Moreover, it has been noted in other ostracode genera (Chivas et al., 1986; Dwyer et al., 1995) that $\mathrm{Mg} / \mathrm{Ca}$-temperature relationships hold at the genus level and that despite the limited data for Loxoconcha sp. and Loxoconcha impressa, this appears to be the case for Loxoconcha .

A second issue pertains to how well the measured temperature represents the actual temperature at the time of shell secretion. The York River samples are well con-
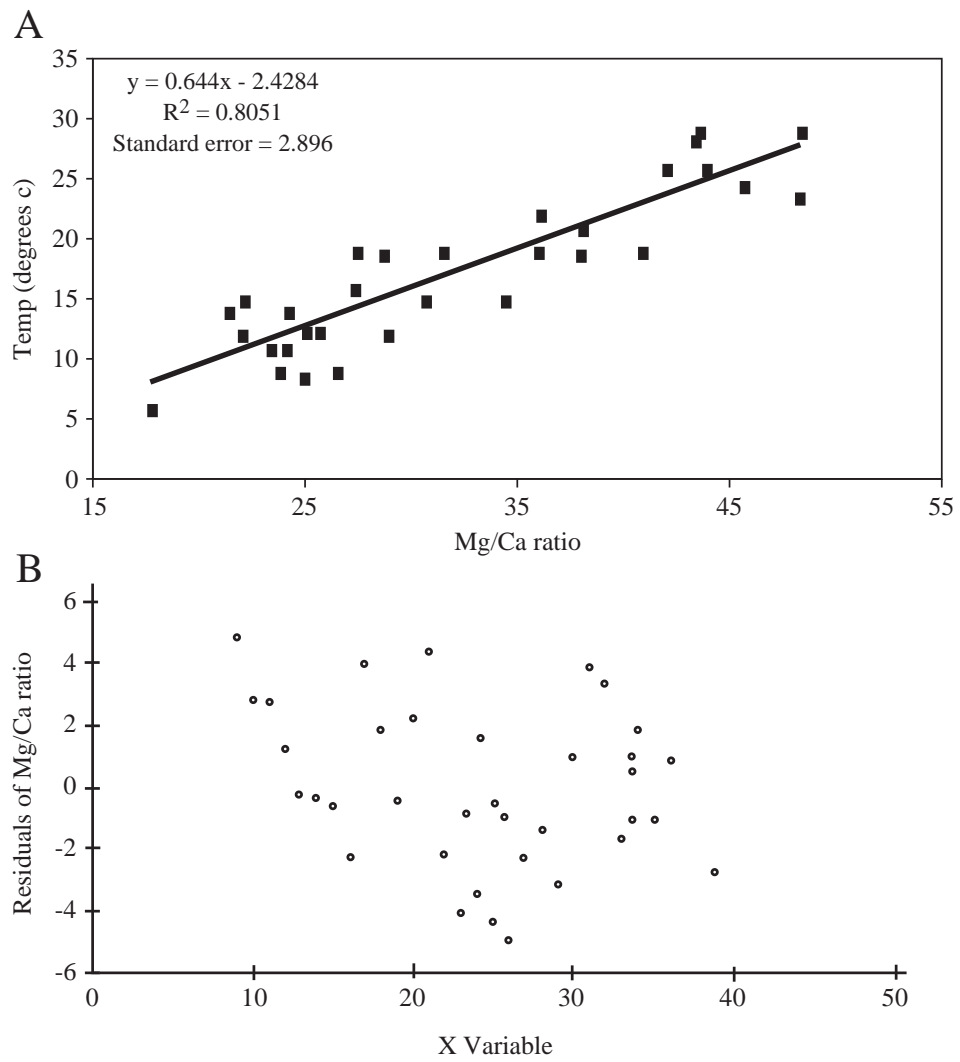

Fig. 11. (A) Linear regression model for $\mathrm{Mg} / \mathrm{Ca}$ ratios in Loxoconcha and water temperature from 36 samples from regions along the eastern United States. (B) Plot of residuals from regression model. See text and Appendix C for discussion. 
strained in terms of seasonal ecology and measured temperature and, as discussed above, there is strong evidence that $T_{\mathrm{m}}$ is either equivalent to, or slightly lower than $T_{\mathrm{s}}$ for most populations. Loxoconcha sp. collected from the main channel of Chesapeake in June and September, 1999 included well-preserved dead adult valves and carapaces. We assume that these Loxoconcha secreted their shells at or near the collection dates and we used mean June and September temperature for each site in the calibration equation obtained from the Chesapeake Bay Program, Annapolis, MD. In the case of Loxoconcha from the continental shelf surface sediment samples, it was assumed that adults grew during the summer months. This assumption seems valid because studies of ostracode biogeography along continental shelf off eastern North America show that these species are limited to the temperate marine climate zone, where there is large seasonal variability in water temperature (Hazel, 1970). If they had evolved to reproduce and survive in colder or warmer temperatures, we would expect them to live in high or lower latitudes, respectively. Summer temperature for each site was obtained from oceanographic sources given in previous ostracode faunal studies using these samples (Valentine, 1971; Cronin and Dowsett, 1990).

In sum, despite some uncertainty of the precise secretion temperature for some samples, the regression in Fig. 11 reflects a first order relationship between temperature and $\mathrm{Mg} / \mathrm{Ca}$ in temperate species of the genus Loxoconcha obtained from a variety of natural estuarine and marine habitats.

\subsection{Intra-shell $\mathrm{Mg} / \mathrm{Ca}$ variability}

Microprobe analyses of Loxoconcha matagordensis from Florida Bay are shown in Figs. 12 and 13. Magnesium/calcium ratios obtained by point measurements taken along transects at two locations in each shell are shown in Fig. 12A and B. Weight percent magnesium and calcium obtained using continuous sweeps across the entire shell width of both shells are shown in Fig. 13A and B. The first point revealed by these plots is that microprobe analyses yield $\mathrm{Mg} / \mathrm{Ca}$ ratios ranging from $\sim 40$ to $65 \mathrm{mmol} / \mathrm{mol}$. These values are near the high end of the range obtained in the DCP-based calibration model discussed above, which seems quite reasonable given the Florida Bay source of the microprobe specimens.

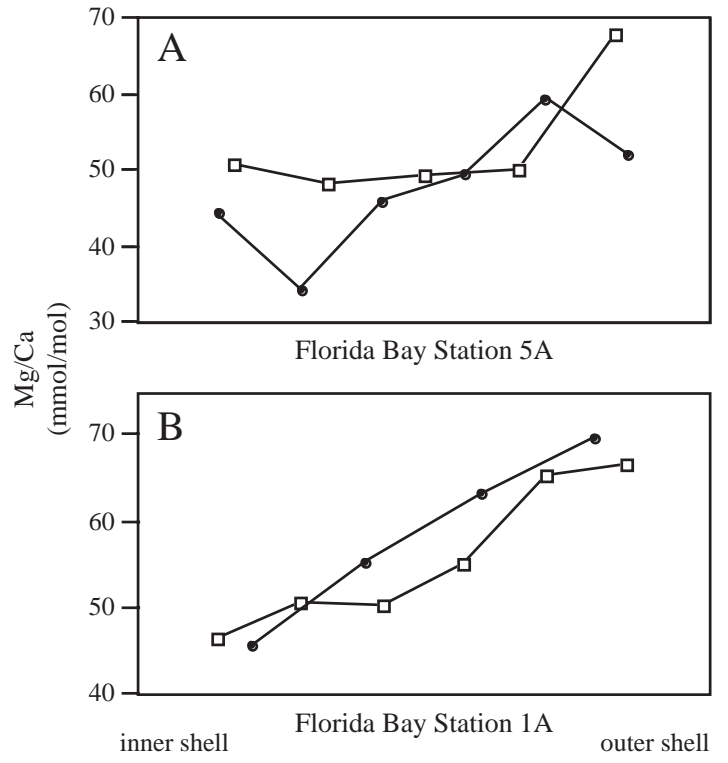

Fig. 12. Microprobe determined $\mathrm{Mg} / \mathrm{Ca}$ ratios in Florida Bay Loxoconcha matagordensis. A and B show transects in two different adult specimens obtained by point measurements.

The second point is that $\mathrm{Mg} / \mathrm{Ca}$ ratios from the inner part of all shells are lower than those from the outer part of the shell. This pattern is seen in both the point and the continuous transects. Intra-shell variability in $\mathrm{Mg} / \mathrm{Ca}$ ratios was also observed by Cadot et al. (1972) in several ostracode genera and in the genus Krithe by Dwyer et al. (2002). It seems to be the result of differing rates of calcification during molting and the amount of organic material incorporated into the shell, but further studies are needed.

\section{Discussion and conclusions}

Most previous studies of ostracode shell chemistry variability have focused either on culturing (Chivas et al., 1986; Majoran et al., 1999; De Deckker et al., 1999), living individuals collected from natural habitats (Cadot and Kaesler, 1977), or mostly dead shells obtained from coretop samples (Corrège, 1993; Dwyer et al., 1995). To our knowledge, the current study is the first seasonal ecological study designed to calibrate $\mathrm{Mg} / \mathrm{Ca}$ ratios using material collected live from natural estuarine/marine habitats at monthly and bimonthly intervals. The results confirm that the seasonal breeding cycle and carapace length-temperature 
relationships observed in the genus Loxoconcha by Kamiya (1988) for Loxoconcha japonica hold for Loxoconcha matagordensis. The carapace size-temperature relationship in Loxoconcha matagordensis may have two explanations. The first is that carapace growth is slower in cooler temperatures due to slower metabolic activity during the molting process. The second may be related to the ecological strategy of Loxoconcha to produce large populations of juveniles for survival during winter months. Kamiya (1988)

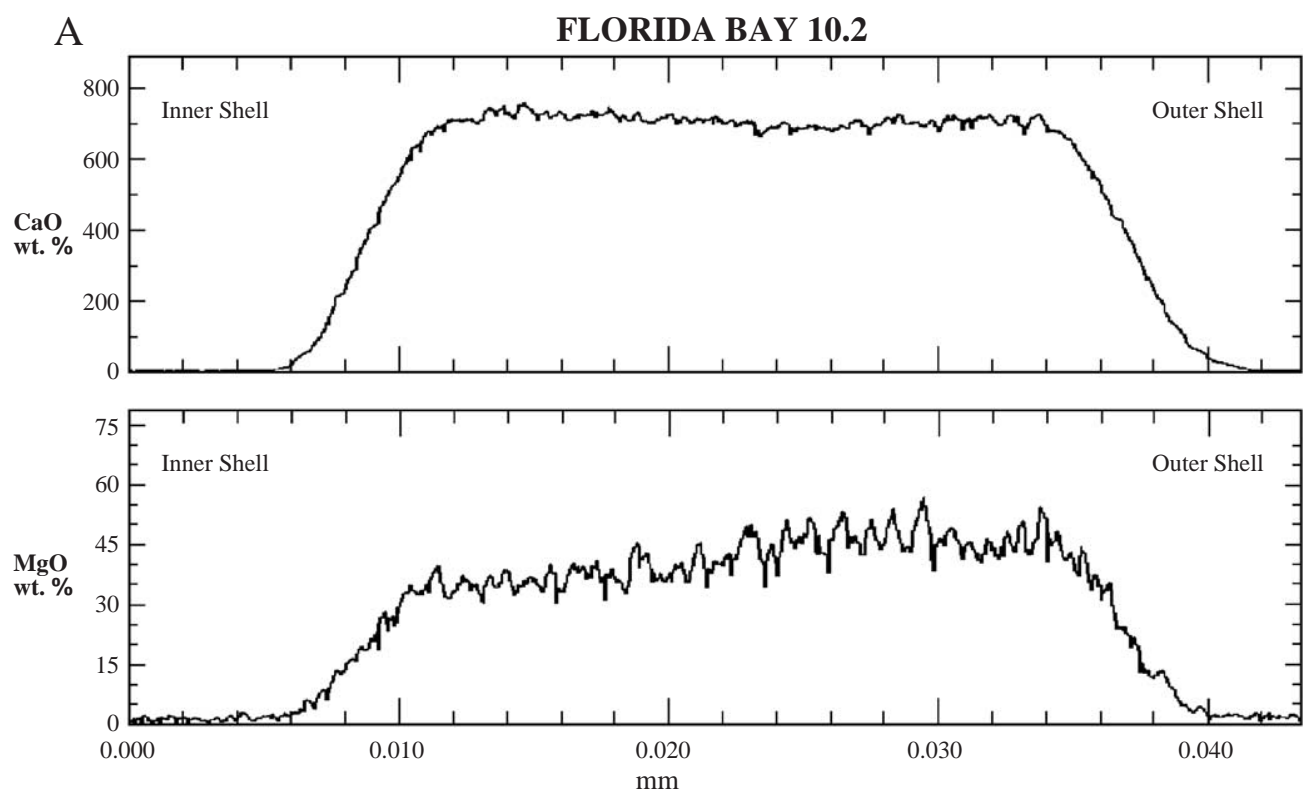

B

FLORIDA BAY 9.1
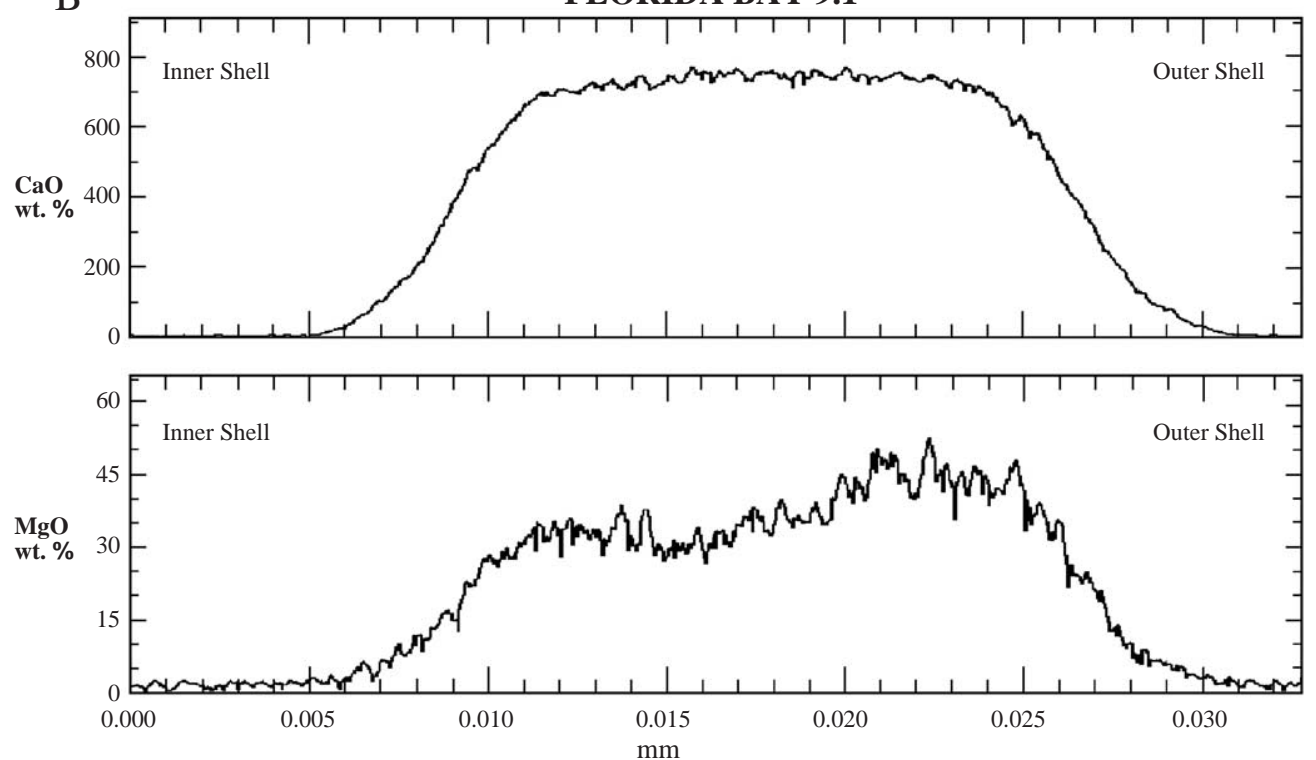

Fig. 13. A and B show the relative concentration of magnesium and calcium (counts/sec) from continuous electron microprobe line scans across entire shells of same two Florida Bay Loxoconcha matagordensis shown in Fig. 12. The right and left portions of the scans record the excitation volume of the electron beam passing into and out of the shell. 

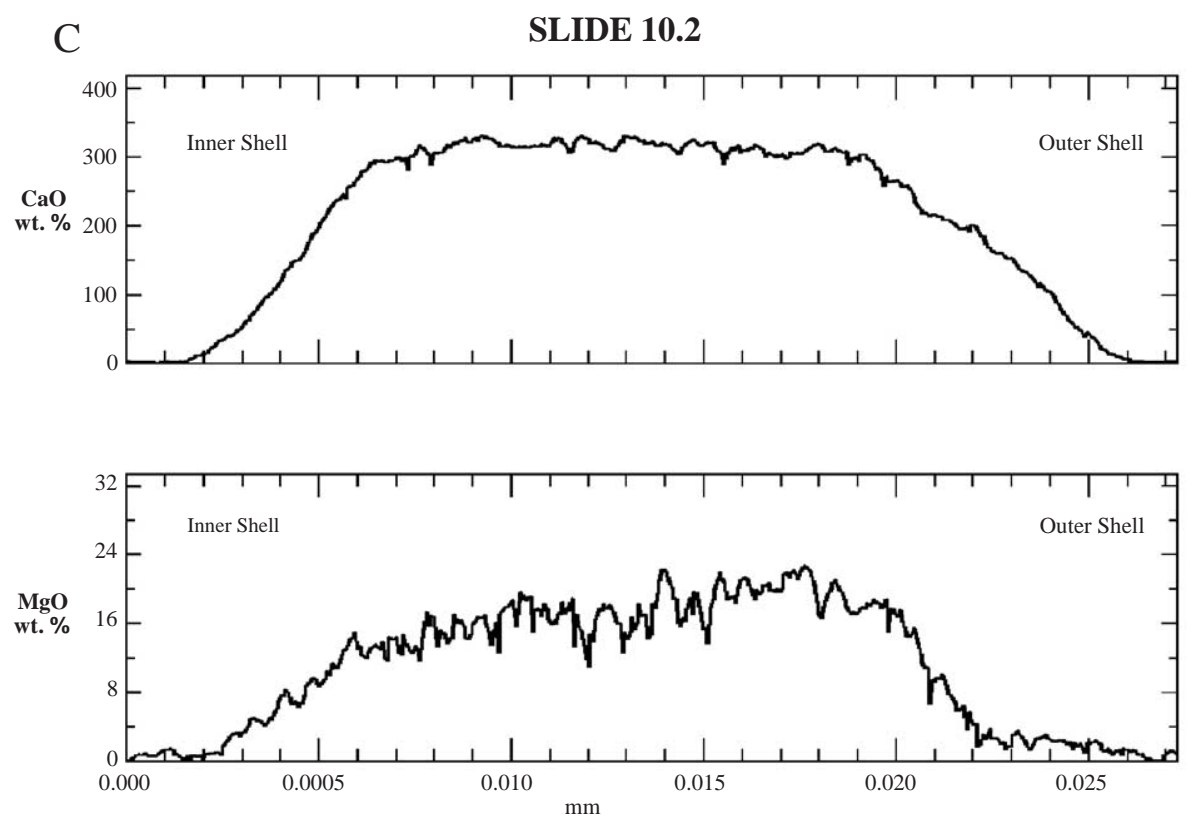

Fig. 13 (continued).

observed summer populations of relatively smallshelled Loxoconcha japonica had abundant, but very small eggs, which require less room in the female carapace. These populations live during a time when Zostera seagrass is dying off during late summer and fall. With reduced Zostera habitat, a large number of offspring may provide a selective advantage to Loxoconcha japonica, for those populations that must survive through the winter until the spring breeding season. Such a situation may also apply to Loxoconcha matagordensis, which has the same general population ecology as Loxoconcha japonica and this idea can be tested with further field and experimental studies.

The apparent lag between shell size and temperature during the spring-early summer populations is probably related to the processes controlling shell coloration and preservation in Loxoconcha. The Loxoconcha carapace becomes "dirty" in three distinct ways: discoloration of epidermal cells, epicutical or the adhesion of organic material to the outer carapace surface. Although we did not distinguish among these factors microscopically, two factors might influence these processes. First, the duration of time between adult shell secretion and collection - the more time, the dirtier the shell becomes. The second, water clarity - the more organic detritus, the dirtier and faster the shell becomes brown. Kamiya (1988) noted that water clarity is relatively good during spring in contrast to more turbid waters during summer and fall. This would mean that even relatively clean individuals collected during spring early summer may have actually secreted their shells 1-2 months prior to collection. Conversely, adult shells secreted during the late summer fall breeding season become dirty relatively quickly due to abundant organic detritus in the water. In general, shell coloration is a useful but not fail-safe method to estimate the age of adult shells and the approximate time since an individual shell was secreted. Even with the uncertainty about precise secretion time, using individuals collected from natural environments has advantages over using cultured specimens because the unnatural environment (i.e. artificial seawater, Kondo et al., 2005) can produce anomalously high $\mathrm{Mg} / \mathrm{Ca}$ ratios and/or weakly calcified carapaces (Dwyer et al., 2002).

The population ecology and the size-temperature relationship in Loxoconcha matagordensis provides a simple means to estimate paleotemperature changes in a series of fossil populations, assuming adults secreted their shells at the same time of year. Fig. 14 provides such an example using male and female populations from two sediment cores from Russell Bank in central Florida Bay. The stratigraphy, age and ostracode faunas 


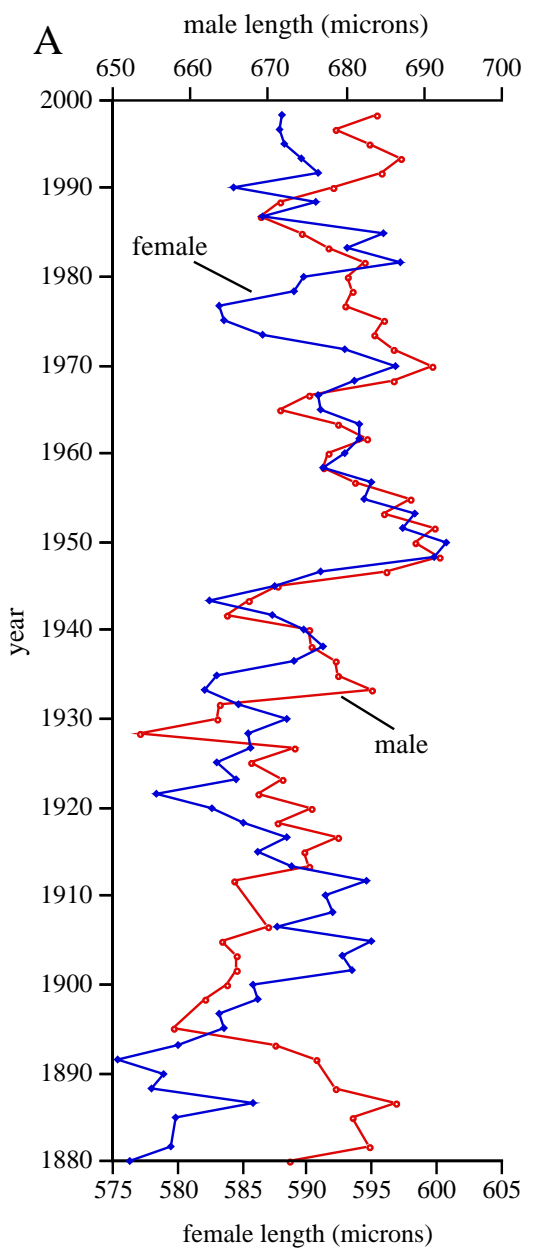

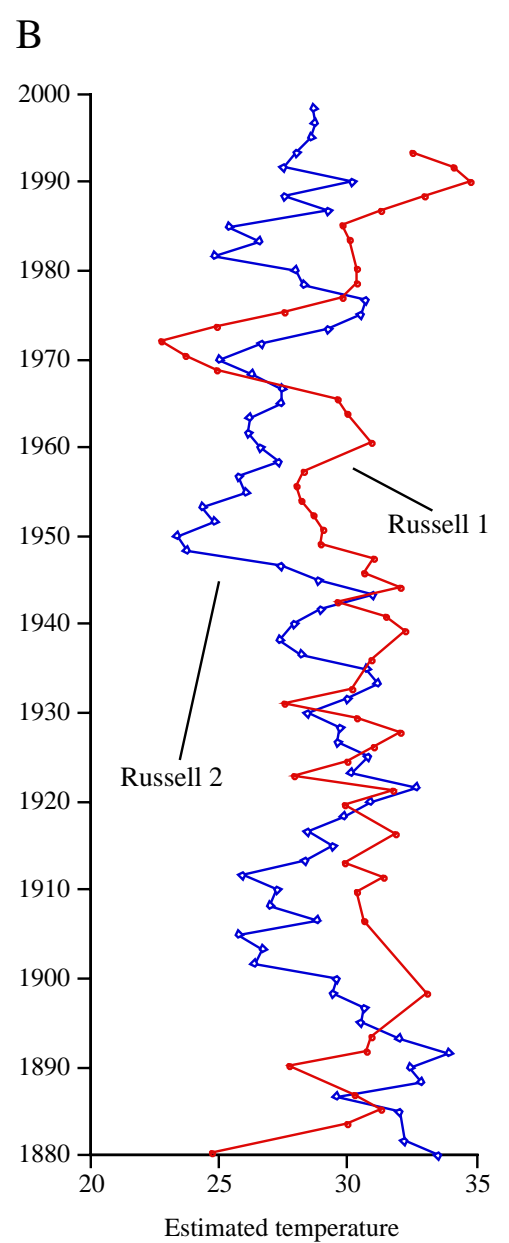

$\left({ }^{\circ} \mathrm{C}\right)$

Fig. 14. (A) Variability of carapace length in male (O) and female (@) Loxoconcha matagordensis from Russell Bank core \#2 for period 1880 1998. (B) Estimated temperature variability in Russell Bank core \#1 $(\bullet)$ and core \#2 $(\diamond)$.

are discussed in detail in Cronin et al. (2001). The figure reveals strikingly similar trends in both male and female populations from the Russell \# 2 core. Further, the size trends from the Russell \# 1 core for females are replicated in the Russell \# 2 core within the limits of the stratigraphy and sampling intervals. Finally, the figure also shows a preliminary plot of temperature history using the size-temperature regression equation given in Fig. 9C. The results indicate that temperature in this part of Florida Bay varied from about 24 to 34 ${ }^{\circ} \mathrm{C}$, estimates that are reasonably close to measured summer temperatures there. In sum, these data provide evidence that the size relationship observed in Loxoconcha male and female populations from the York
River holds true for Florida Bay populations. Further studies of this simple indicator may prove useful as a means of estimating past water temperature variability.

The ecological data obtained on Loxoconcha also aid in the interpretation of the $\mathrm{Mg} / \mathrm{Ca}$-temperature relationships from the standpoint of distinguishing between time of adult shell secretion and collection time. At first examination, the match between water temperature and $\mathrm{Mg} / \mathrm{Ca}$ ratios in Fig. 10A seems excellent, suggesting adults secreted their adult shell very close to the time of collection. Such an interpretation, however, requires qualification in two respects. First, adults collected in winter and early spring, 1999 (February-May) most likely represent individual that grew 
their adult shells during the prior fall, and thus at a slightly higher temperature than those of the FebruaryMay collection date. The dominance of extremely dirty shells collected during these months as well as the patterns observed in Loxoconcha japonica support this view. Second, if adult Loxoconcha matagordensis collected in May-July actually secreted their shells 1-2 months earlier than the collection date, as suggested by the size-temperature relationship, then the temperature at the time of secretion of these adults may have been slightly lower than that measured. Although these problems do not diminish the value of the $\mathrm{Mg} / \mathrm{Ca}$ temperature relationship, it is likely that an improved $\mathrm{Mg} / \mathrm{Ca}$ temperature calibration might be obtained with additional material collected bimonthly for which shell preservation is carefully evaluated. Studies of Loxoconcha matagordensis collected bimonthly from
Dameron Marsh in Chesapeake Bay are underway to refine the calibration using adults secreted during the spring/summer season.

\section{Acknowledgments}

We are extremely grateful to Drs. K. Moore, Dr. R. Orth and B. Berry-Niekirk and their staff at the Virginia Institute of Marine Science for collection of York River seagrass samples under the auspices of the Virginia Nearshore SAV Habitat Monitoring Program. Dr. Dan Dauer of Old Dominion University provided assistance with sampling the deep Chesapeake Channel. Dr. G. Tanaka, J. Repetski, D. Willard and an anonymous reviewer provided useful comments of the manuscript.

\section{Appendix A}

Size data for Loxoconcha matagordensis from Chesapeake Bay: Males

\begin{tabular}{|c|c|c|c|c|c|c|c|c|c|}
\hline Site & Date & Color & Age & $\begin{array}{l}\text { Size } \\
(\mu \mathrm{m})\end{array}$ & Sex & $\begin{array}{l}\text { Mean size } \\
\text { Guinea }(\mu \mathrm{m})\end{array}$ & $\begin{array}{l}\text { Mean size } \\
\text { Goodwin }(\mu \mathrm{m})\end{array}$ & $\begin{array}{l}\text { Mean size } \\
\text { Dameron }(\mu \mathrm{m})\end{array}$ & $\begin{array}{l}\text { Grand mean } \\
(\mu \mathrm{m})\end{array}$ \\
\hline Goodwin Island & 2/9/1999 & Brown & Old & 716 & M & & 710 & & 707.6666667 \\
\hline Goodwin Island & 2/9/1999 & Brown & Old & 704 & M & & & & \\
\hline Guinea Marsh & 2/9/1999 & Trans-brown & Intermediate & 726 & M & 706.5 & & & \\
\hline Guinea Marsh & 2/9/1999 & Trans-brown & Intermediate & 700 & M & & & & \\
\hline Guinea Marsh & 2/9/1999 & Trans-brown & Intermediate & 730 & M & & & & \\
\hline Guinea Marsh & 2/9/1999 & Brown & Old & 670 & M & & & & \\
\hline Guinea Marsh & 4/27/1999 & Transparent & New & 706 & M & 705.1428571 & & & 705.1428571 \\
\hline Guinea Marsh & 4/27/1999 & Brown & Old & 720 & M & & & & \\
\hline Guinea Marsh & 4/27/1999 & Brown & Old & 704 & M & & & & \\
\hline Guinea Marsh & 4/27/1999 & Brown & Old & 720 & M & & & & \\
\hline Guinea Marsh & $4 / 27 / 1999$ & Brown & Old & 690 & M & & & & \\
\hline Guinea Marsh & $4 / 27 / 1999$ & Brown & Old & 696 & M & & & & \\
\hline Guinea Marsh & 4/27/1999 & Brown & Old & 700 & M & & & & \\
\hline Goodwin Island & $5 / 12 / 1999$ & Brown & Old & 664 & M & & 664 & & 689.5 \\
\hline Guinea Marsh & $5 / 12 / 1999$ & Transparent & New & 726 & M & 698 & & & \\
\hline Guinea Marsh & $5 / 12 / 1999$ & Brown & Old & 682 & M & 684 & & & \\
\hline Guinea Marsh & $5 / 12 / 1999$ & Brown & Old & 686 & M & & & & \\
\hline Goodwin Island & $6 / 22 / 1999$ & Trans-brown & Intermediate & 724 & M & & 707.7142857 & & 714.1714286 \\
\hline Goodwin Island & $6 / 22 / 1999$ & Transparent & New & 682 & M & & & & \\
\hline Goodwin Island & $6 / 22 / 1999$ & Transparent & New & 690 & M & & & & \\
\hline Goodwin Island & $6 / 22 / 1999$ & Transparent & New & 700 & M & & & & \\
\hline Goodwin Island & $6 / 22 / 1999$ & Brown & Old & 720 & M & & & & \\
\hline Goodwin Island & $6 / 22 / 1999$ & Brown & Old & 718 & M & & & & \\
\hline Goodwin Island & $6 / 22 / 1999$ & Brown & Old & 720 & M & & & & \\
\hline Guinea Marsh & $6 / 22 / 1999$ & Trans-brown & Intermediate & 712 & M & 715.7857143 & & & \\
\hline Guinea Marsh & $6 / 22 / 1999$ & Trans-brown & Intermediate & 698 & M & & & & \\
\hline Guinea Marsh & $6 / 22 / 1999$ & Transparent & New & 684 & M & & & & \\
\hline
\end{tabular}


Appendix A (continued)

\begin{tabular}{|c|c|c|c|c|c|c|c|c|c|}
\hline Site & Date & Color & Age & $\begin{array}{l}\text { Size } \\
(\mu \mathrm{m})\end{array}$ & Sex & $\begin{array}{l}\text { Mean size } \\
\text { Guinea }(\mu \mathrm{m})\end{array}$ & $\begin{array}{l}\text { Mean size } \\
\text { Goodwin }(\mu \mathrm{m})\end{array}$ & $\begin{array}{l}\text { Mean size } \\
\text { Dameron }(\mu \mathrm{m})\end{array}$ & $\begin{array}{l}\text { Grand mean } \\
(\mu \mathrm{m})\end{array}$ \\
\hline Guinea Marsh & $6 / 22 / 1999$ & Transparent & New & 704 & M & & & & \\
\hline Guinea Marsh & $6 / 22 / 1999$ & Transparent & New & 702 & M & & & & \\
\hline Guinea Marsh & $6 / 22 / 1999$ & Transparent & New & 720 & M & & & & \\
\hline Guinea Marsh & $6 / 22 / 1999$ & Brown & Old & 720 & M & & & & \\
\hline Guinea Marsh & 6/22/1999 & Brown & Old & 706 & M & & & & \\
\hline Guinea Marsh & $6 / 22 / 1999$ & Brown & Old & 720 & M & & & & \\
\hline Guinea Marsh & $6 / 22 / 1999$ & Brown & Old & 714 & M & & & & \\
\hline Guinea Marsh & $6 / 22 / 1999$ & Brown & Old & 736 & M & & & & \\
\hline Guinea Marsh & $6 / 22 / 1999$ & Brown & Old & 734 & M & & & & \\
\hline Guinea Marsh & $6 / 22 / 1999$ & Brown & Old & 724 & M & & & & \\
\hline Guinea Marsh & 6/22/1999 & Brown & Old & 726 & M & & & & \\
\hline Guinea Marsh & $6 / 22 / 1999$ & Brown & Old & 706 & M & & & & \\
\hline Guinea Marsh & $6 / 22 / 1999$ & Brown & Old & 712 & M & & & & \\
\hline Guinea Marsh & 6/22/1999 & Brown & Old & 684 & M & & & & \\
\hline Guinea Marsh & $6 / 22 / 1999$ & Brown & Old & 706 & M & & & & \\
\hline Guinea Marsh & $6 / 22 / 1999$ & Brown & Old & 700 & M & & & & \\
\hline Guinea Marsh & 6/22/1999 & Brown & Old & 726 & M & & & & \\
\hline Guinea Marsh & 6/22/1999 & Brown & Old & 724 & M & & & & \\
\hline Guinea Marsh & $6 / 22 / 1999$ & Brown & Old & 730 & M & & & & \\
\hline Guinea Marsh & $6 / 22 / 1999$ & Brown & Old & 740 & M & & & & \\
\hline Guinea Marsh & 6/22/1999 & Brown & Old & 738 & M & & & & \\
\hline Guinea Marsh & $6 / 22 / 1999$ & Brown & Old & 722 & M & & & & \\
\hline Guinea Marsh & $6 / 22 / 1999$ & Brown & Old & 706 & M & & & & \\
\hline Guinea Marsh & 6/22/1999 & Yellow-brown & Old & 730 & M & & & & \\
\hline Guinea Marsh & $6 / 22 / 1999$ & Very brown & Very old & 718 & M & & & & \\
\hline Goodwin Island & 7/6/1999 & Trans-brown & Intermediate & 696 & M & & 704 & & 708.727273 \\
\hline Goodwin Island & $7 / 6 / 1999$ & Brown & Old & 694 & M & & & & \\
\hline Goodwin Island & 7/6/1999 & Yellow-brown & Old & 706 & $\mathrm{M}$ & & & & \\
\hline Goodwin Island & 7/6/1999 & Yellow-brown & Old & 720 & M & & & & \\
\hline Guinea Marsh & 7/6/1999 & Trans-brown & Intermediate & 706 & M & 711.4285714 & & & \\
\hline Guinea Marsh & 7/6/1999 & Trans-brown & Intermediate & 702 & $\mathrm{M}$ & & & & \\
\hline Guinea Marsh & 7/6/1999 & Transparent & New & 710 & M & & & & \\
\hline Guinea Marsh & 7/6/1999 & Transparent & New & 716 & M & & & & \\
\hline Guinea Marsh & 7/6/1999 & Transparent & New & 708 & $\mathrm{M}$ & & & & \\
\hline Guinea Marsh & 7/6/1999 & Brown & Old & 732 & M & & & & \\
\hline Guinea Marsh & 7/6/1999 & Brown & Old & 706 & M & & & & \\
\hline Goodwin Island & 7/20/1999 & Trans-brown & Intermediate & 704 & M & & 711 & & 706.350877 \\
\hline Goodwin Island & 7/20/1999 & Trans-brown & Intermediate & 702 & M & & & & \\
\hline Goodwin Island & 7/20/1999 & Trans-brown & Intermediate & 698 & $\mathrm{M}$ & & & & \\
\hline Goodwin Island & 7/20/1999 & Trans-brown & Intermediate & 708 & M & & & & \\
\hline Goodwin Island & 7/20/1999 & Trans-brown & Intermediate & 700 & M & & & & \\
\hline Goodwin Island & 7/20/1999 & Transparent & New & 692 & $\mathrm{M}$ & & & & \\
\hline Goodwin Island & 7/20/1999 & Brown & Old & 704 & M & & & & \\
\hline Goodwin Island & 7/20/1999 & Brown & Old & 720 & M & & & & \\
\hline Goodwin Island & 7/20/1999 & Brown & Old & 706 & $\mathrm{M}$ & & & & \\
\hline Goodwin Island & 7/20/1999 & Opaque & Old & 710 & M & & & & \\
\hline Goodwin Island & 7/20/1999 & Yellow-opaque & Old & 726 & M & & & & \\
\hline Goodwin Island & 7/20/1999 & Yellow-opaque & Old & 696 & M & & & & \\
\hline Goodwin Island & 7/20/1999 & Yellow-opaque & Old & 722 & M & & & & \\
\hline Goodwin Island & 7/20/1999 & Yellow-opaque & Old & 722 & M & & & & \\
\hline Goodwin Island & 7/20/1999 & Brown-opaque & Very old & 720 & M & & & & \\
\hline Goodwin Island & 7/20/1999 & Brown-opaque & Very old & 746 & M & & & & \\
\hline Guinea Marsh & 7/20/1999 & Trans-brown & Intermediate & 714 & M & 704.5365854 & & & \\
\hline
\end{tabular}


Appendix A (continued)

\begin{tabular}{|c|c|c|c|c|c|c|c|c|c|}
\hline Site & Date & Color & Age & $\begin{array}{l}\text { Size } \\
(\mu \mathrm{m})\end{array}$ & Sex & $\begin{array}{l}\text { Mean size } \\
\text { Guinea }(\mu \mathrm{m})\end{array}$ & $\begin{array}{l}\text { Mean size } \\
\text { Goodwin }(\mu \mathrm{m})\end{array}$ & $\begin{array}{l}\text { Mean size } \\
\text { Dameron }(\mu \mathrm{m})\end{array}$ & $\begin{array}{l}\text { Grand mean } \\
(\mu \mathrm{m})\end{array}$ \\
\hline Guinea Marsh & 7/20/1999 & Trans-brown & Intermediate & 704 & M & & & & \\
\hline Guinea Marsh & 7/20/1999 & Trans-brown & Intermediate & 700 & M & & & & \\
\hline Guinea Marsh & 7/20/1999 & Trans-brown & Intermediate & 704 & M & & & & \\
\hline Guinea Marsh & 7/20/1999 & Trans-brown & Intermediate & 716 & M & & & & \\
\hline Guinea Marsh & $7 / 20 / 1999$ & Trans-brown & Intermediate & 706 & M & & & & \\
\hline Guinea Marsh & 7/20/1999 & Trans-brown & Intermediate & 708 & M & & & & \\
\hline Guinea Marsh & 7/20/1999 & Trans-brown & Intermediate & 704 & M & & & & \\
\hline Guinea Marsh & $7 / 20 / 1999$ & Trans-brown & Intermediate & 704 & M & & & & \\
\hline Guinea Marsh & 7/20/1999 & Trans-brown & Intermediate & 690 & M & & & & \\
\hline Guinea Marsh & 7/20/1999 & Trans-brown & Intermediate & 700 & M & & & & \\
\hline Guinea Marsh & 7/20/1999 & Trans-brown & Intermediate & 692 & M & & & & \\
\hline Guinea Marsh & $7 / 20 / 1999$ & Trans-brown & Intermediate & 700 & M & & & & \\
\hline Guinea Marsh & $7 / 20 / 1999$ & Trans-brown & Intermediate & 710 & M & & & & \\
\hline Guinea Marsh & 7/20/1999 & Trans-brown & Intermediate & 714 & M & & & & \\
\hline Guinea Marsh & $7 / 20 / 1999$ & Transparent & New & 700 & M & & & & \\
\hline Guinea Marsh & $7 / 20 / 1999$ & Transparent & New & 706 & M & & & & \\
\hline Guinea Marsh & 7/20/1999 & Transparent & New & 704 & M & & & & \\
\hline Guinea Marsh & $7 / 20 / 1999$ & Transparent & New & 702 & M & & & & \\
\hline Guinea Marsh & $7 / 20 / 1999$ & Transparent & New & 700 & M & & & & \\
\hline Guinea Marsh & $7 / 20 / 1999$ & Transparent & New & 706 & M & & & & \\
\hline Guinea Marsh & $7 / 20 / 1999$ & Transparent & New & 706 & M & & & & \\
\hline Guinea Marsh & $7 / 20 / 1999$ & Transparent & New & 690 & M & & & & \\
\hline Guinea Marsh & $7 / 20 / 1999$ & Transparent & New & 702 & M & & & & \\
\hline Guinea Marsh & $7 / 20 / 1999$ & Transparent & New & 706 & M & & & & \\
\hline Guinea Marsh & 7/20/1999 & Transparent & New & 686 & M & & & & \\
\hline Guinea Marsh & $7 / 20 / 1999$ & Transparent & New & 700 & M & & & & \\
\hline Guinea Marsh & $7 / 20 / 1999$ & Transparent & New & 694 & M & & & & \\
\hline Guinea Marsh & 7/20/1999 & Transparent & New & 710 & M & & & & \\
\hline Guinea Marsh & $7 / 20 / 1999$ & Brown & Old & 706 & M & & & & \\
\hline Guinea Marsh & $7 / 20 / 1999$ & Brown & Old & 702 & M & & & & \\
\hline Guinea Marsh & 7/20/1999 & Brown & Old & 712 & M & & & & \\
\hline Guinea Marsh & 7/20/1999 & Brown & Old & 708 & M & & & & \\
\hline Guinea Marsh & 7/20/1999 & Brown & Old & 704 & M & & & & \\
\hline Guinea Marsh & $7 / 20 / 1999$ & Brown & Old & 698 & M & & & & \\
\hline Guinea Marsh & $7 / 20 / 1999$ & Brown & Old & 708 & M & & & & \\
\hline Guinea Marsh & 7/20/1999 & Brown & Old & 708 & M & & & & \\
\hline Guinea Marsh & $7 / 20 / 1999$ & Brown & Old & 720 & M & & & & \\
\hline Guinea Marsh & $7 / 20 / 1999$ & Brown-opaque & Very old & 700 & M & & & & \\
\hline Guinea Marsh & 7/20/1999 & Brown-opaque & Very old & 720 & M & & & & \\
\hline Guinea Marsh & $7 / 20 / 1999$ & Brown-opaque & Very old & 722 & M & & & & \\
\hline Goodwin Island & 8/3/1999 & Transparent & New & 700 & M & & 706 & & 707.625 \\
\hline Goodwin Island & 8/3/1999 & Transparent & New & 704 & M & & & & \\
\hline Goodwin Island & 8/3/1999 & Transparent & New & 720 & M & & & & \\
\hline Goodwin Island & 8/3/1999 & Transparent & New & 708 & M & & & & \\
\hline Goodwin Island & 8/3/1999 & Brown & Old & 720 & M & & & & \\
\hline Goodwin Island & 8/3/1999 & Brown & Old & 716 & M & & & & \\
\hline Goodwin Island & 8/3/1999 & Brown & Old & 702 & M & & & & \\
\hline Goodwin Island & 8/3/1999 & Brown & Old & 700 & M & & & & \\
\hline Goodwin Island & 8/3/1999 & Brown-white & Old & 690 & M & & & & \\
\hline Goodwin Island & 8/3/1999 & Brown-white & Old & 700 & M & & & & \\
\hline Guinea Marsh & 8/3/1999 & Trans-brown & Intermediate & 700 & M & 708.3636364 & & & \\
\hline Guinea Marsh & 8/3/1999 & Trans-brown & Intermediate & 718 & M & & & & \\
\hline Guinea Marsh & 8/3/1999 & Trans-brown & Intermediate & 706 & M & & & & \\
\hline
\end{tabular}


Appendix A (continued)

\begin{tabular}{|c|c|c|c|c|c|c|c|c|c|}
\hline Site & Date & Color & Age & $\begin{array}{l}\text { Size } \\
(\mu \mathrm{m})\end{array}$ & Sex & $\begin{array}{l}\text { Mean size } \\
\text { Guinea }(\mu \mathrm{m})\end{array}$ & $\begin{array}{l}\text { Mean size } \\
\text { Goodwin }(\mu \mathrm{m})\end{array}$ & $\begin{array}{l}\text { Mean size } \\
\text { Dameron }(\mu \mathrm{m})\end{array}$ & $\begin{array}{l}\text { Grand mean } \\
(\mu \mathrm{m})\end{array}$ \\
\hline Guinea Marsh & 8/3/1999 & Trans-brown & Intermediate & 722 & M & & & & \\
\hline Guinea Marsh & 8/3/1999 & Trans-brown & Intermediate & 704 & M & & & & \\
\hline Guinea Marsh & 8/3/1999 & Trans-brown & Intermediate & 698 & M & & & & \\
\hline Guinea Marsh & 8/3/1999 & Transparent & New & 692 & M & & & & \\
\hline Guinea Marsh & 8/3/1999 & Transparent & New & 720 & M & & & & \\
\hline Guinea Marsh & 8/3/1999 & Transparent & New & 702 & M & & & & \\
\hline Guinea Marsh & 8/3/1999 & Transparent & New & 714 & M & & & & \\
\hline Guinea Marsh & 8/3/1999 & Transparent & New & 696 & M & & & & \\
\hline Guinea Marsh & 8/3/1999 & Transparent & New & 692 & M & & & & \\
\hline Guinea Marsh & 8/3/1999 & Transparent & New & 704 & M & & & & \\
\hline Guinea Marsh & 8/3/1999 & Transparent & New & 682 & M & & & & \\
\hline Guinea Marsh & 8/3/1999 & Brown & Old & 706 & M & & & & \\
\hline Guinea Marsh & 8/3/1999 & Brown & Old & 730 & M & & & & \\
\hline Guinea Marsh & 8/3/1999 & Brown & Old & 724 & M & & & & \\
\hline Guinea Marsh & 8/3/1999 & Brown & Old & 726 & M & & & & \\
\hline Guinea Marsh & 8/3/1999 & Brown & Old & 710 & M & & & & \\
\hline Guinea Marsh & 8/3/1999 & Brown-opaque & Very old & 720 & M & & & & \\
\hline Guinea Marsh & 8/3/1999 & Brown-opaque & Very old & 704 & M & & & & \\
\hline Guinea Marsh & 8/3/1999 & Brown-opaque & Very old & 714 & M & & & & \\
\hline Goodwin Island & $8 / 17 / 1999$ & Trans-brown & Intermediate & 692 & M & & 693.7894737 & & 695.0909091 \\
\hline Goodwin Island & $8 / 17 / 1999$ & Trans-brown & Intermediate & 682 & M & & & & \\
\hline Goodwin Island & $8 / 17 / 1999$ & Trans-brown & Intermediate & 706 & M & & & & \\
\hline Goodwin Island & $8 / 17 / 1999$ & Trans-white & Intermediate/old & 678 & M & & & & \\
\hline Goodwin Island & $8 / 17 / 1999$ & Transparent & New & 686 & M & & & & \\
\hline Goodwin Island & $8 / 17 / 1999$ & Transparent & New & 680 & M & & & & \\
\hline Goodwin Island & $8 / 17 / 1999$ & Transparent & New & 688 & M & & & & \\
\hline Goodwin Island & $8 / 17 / 1999$ & Transparent & New & 704 & M & & & & \\
\hline Goodwin Island & $8 / 17 / 1999$ & Brown & Old & 700 & M & & & & \\
\hline Goodwin Island & $8 / 17 / 1999$ & Brown & Old & 682 & M & & & & \\
\hline Goodwin Island & $8 / 17 / 1999$ & Brown & Old & 706 & M & & & & \\
\hline Goodwin Island & $8 / 17 / 1999$ & Brown & Old & 700 & M & & & & \\
\hline Goodwin Island & $8 / 17 / 1999$ & Brown & Old & 688 & M & & & & \\
\hline Goodwin Island & $8 / 17 / 1999$ & Brown & Old & 698 & M & & & & \\
\hline Goodwin Island & $8 / 17 / 1999$ & Brown & Old & 706 & M & & & & \\
\hline Goodwin Island & $8 / 17 / 1999$ & Brown-opaque & Very old & 702 & M & & & & \\
\hline Goodwin Island & $8 / 17 / 1999$ & Brown-opaque & Very old & 700 & M & & & & \\
\hline Goodwin Island & $8 / 17 / 1999$ & Brown-opaque & Very old & 702 & M & & & & \\
\hline Goodwin Island & $8 / 17 / 1999$ & $\mathrm{n} / \mathrm{a}$ & $\mathrm{n} / \mathrm{a}$ & 682 & M & & & & \\
\hline Guinea Marsh & $8 / 17 / 1999$ & Trans-brown & Intermediate & 710 & M & 703.3333333 & & & \\
\hline Guinea Marsh & $8 / 17 / 1999$ & Trans-brown & Intermediate & 706 & M & & & & \\
\hline Guinea Marsh & $8 / 17 / 1999$ & Transparent & New & 694 & M & & & & \\
\hline Goodwin Island & 9/14/1999 & Brown & Old & 682 & M & & 682 & & 680.5333333 \\
\hline Goodwin Island & 9/14/1999 & Brown & Old & 680 & M & & & & \\
\hline Goodwin Island & $9 / 14 / 1999$ & Brown-opaque & Very old & 684 & M & & & & \\
\hline Guinea Marsh & 9/14/1999 & Trans-brown & Intermediate & 690 & M & 680.1666667 & & & \\
\hline Guinea Marsh & $9 / 14 / 1999$ & Trans-brown & Intermediate & 676 & M & & & & \\
\hline Guinea Marsh & $9 / 14 / 1999$ & Trans-brown & Intermediate & 680 & M & & & & \\
\hline Guinea Marsh & 9/14/1999 & Transparent & New & 696 & M & & & & \\
\hline Guinea Marsh & $9 / 14 / 1999$ & Transparent & New & 664 & M & & & & \\
\hline Guinea Marsh & 9/14/1999 & Brown & Old & 660 & M & & & & \\
\hline Guinea Marsh & 9/14/1999 & Brown & Old & 682 & M & & & & \\
\hline Guinea Marsh & 9/14/1999 & Brown & Old & 656 & M & & & & \\
\hline Guinea Marsh & 9/14/1999 & Brown & Old & 694 & M & & & & \\
\hline
\end{tabular}




\begin{tabular}{|c|c|c|c|c|c|c|c|c|c|}
\hline Site & Date & Color & Age & $\begin{array}{l}\text { Size } \\
(\mu \mathrm{m})\end{array}$ & Sex & $\begin{array}{l}\text { Mean size } \\
\text { Guinea }(\mu \mathrm{m})\end{array}$ & $\begin{array}{l}\text { Mean size } \\
\text { Goodwin }(\mu \mathrm{m})\end{array}$ & $\begin{array}{l}\text { Mean size } \\
\text { Dameron }(\mu \mathrm{m})\end{array}$ & $\begin{array}{l}\text { Grand mean } \\
(\mu \mathrm{m})\end{array}$ \\
\hline Guinea Marsh & $9 / 14 / 1999$ & Brown & Old & 674 & M & & & & \\
\hline Guinea Marsh & 9/14/1999 & Brown & Old & 680 & M & & & & \\
\hline Guinea Marsh & $9 / 14 / 1999$ & Brown-opaque & Very old & 710 & M & & & & \\
\hline Guinea Marsh & $10 / 15 / 1999$ & Trans-brown & Intermediate & 696 & M & 702.7272727 & & & 702.72727273 \\
\hline Guinea Marsh & $10 / 15 / 1999$ & Trans-brown & Intermediate & 702 & M & & & & \\
\hline Guinea Marsh & $10 / 15 / 1999$ & Trans-brown & Intermediate & 702 & M & & & & \\
\hline Guinea Marsh & $10 / 15 / 1999$ & Trans-brown & Intermediate & 696 & M & & & & \\
\hline Guinea Marsh & $10 / 15 / 1999$ & Transparent & New & 712 & M & & & & \\
\hline Guinea Marsh & $10 / 15 / 1999$ & Transparent & New & 710 & M & & & & \\
\hline Guinea Marsh & $10 / 15 / 1999$ & Transparent & New & 704 & M & & & & \\
\hline Guinea Marsh & $10 / 15 / 1999$ & Brown & Old & 700 & M & & & & \\
\hline Guinea Marsh & $10 / 15 / 1999$ & Brown & Old & 702 & M & & & & \\
\hline Guinea Marsh & $10 / 15 / 1999$ & Brown & Old & 698 & M & & & & \\
\hline Guinea Marsh & $10 / 15 / 1999$ & Brown & Old & 708 & M & & & & \\
\hline Goodwin Island & 11/9/1999 & Trans-brown & Intermediate & 704 & M & & 711.4 & & 711.2698413 \\
\hline Goodwin Island & $11 / 9 / 1999$ & Trans-white & Intermediate/old & 720 & M & & & & \\
\hline Goodwin Island & $11 / 9 / 1999$ & Trans-white & Intermediate/old & 720 & M & & & & \\
\hline Goodwin Island & $11 / 9 / 1999$ & Trans-white & Intermediate/old & 724 & M & & & & \\
\hline Goodwin Island & $11 / 9 / 1999$ & Trans-white & Intermediate/old & 700 & M & & & & \\
\hline Goodwin Island & $11 / 9 / 1999$ & Transparent & New & 700 & M & & & & \\
\hline Goodwin Island & $11 / 9 / 1999$ & Transparent & New & 720 & M & & & & \\
\hline Goodwin Island & $11 / 9 / 1999$ & Transparent & New & 722 & M & & & & \\
\hline Goodwin Island & 11/9/1999 & Transparent & New & 720 & M & & & & \\
\hline Goodwin Island & $11 / 9 / 1999$ & Brown-white & Old & 684 & M & & & & \\
\hline Guinea Marsh & $11 / 9 / 1999$ & Trans-brown & Intermediate & 718 & M & 711.245283 & & & \\
\hline Guinea Marsh & 11/9/1999 & Trans-brown & Intermediate & 680 & M & & & & \\
\hline Guinea Marsh & $11 / 9 / 1999$ & Trans-brown & Intermediate & 720 & M & & & & \\
\hline Guinea Marsh & $11 / 9 / 1999$ & Transparent & New & 702 & M & & & & \\
\hline Guinea Marsh & 11/9/1999 & Transparent & New & 714 & M & & & & \\
\hline Guinea Marsh & $11 / 9 / 1999$ & Transparent & New & 690 & M & & & & \\
\hline Guinea Marsh & $11 / 9 / 1999$ & Transparent & New & 716 & M & & & & \\
\hline Guinea Marsh & 11/9/1999 & Transparent & New & 700 & M & & & & \\
\hline Guinea Marsh & $11 / 9 / 1999$ & Transparent & New & 700 & M & & & & \\
\hline Guinea Marsh & $11 / 9 / 1999$ & Transparent & New & 708 & M & & & & \\
\hline Guinea Marsh & $11 / 9 / 1999$ & Transparent & New & 712 & M & & & & \\
\hline Guinea Marsh & $11 / 9 / 1999$ & Transparent & New & 724 & M & & & & \\
\hline Guinea Marsh & $11 / 9 / 1999$ & Transparent & New & 710 & M & & & & \\
\hline Guinea Marsh & $11 / 9 / 1999$ & Transparent & New & 700 & M & & & & \\
\hline Guinea Marsh & $11 / 9 / 1999$ & Transparent & New & 722 & M & & & & \\
\hline Guinea Marsh & $11 / 9 / 1999$ & Transparent & New & 720 & M & & & & \\
\hline Guinea Marsh & $11 / 9 / 1999$ & Transparent & New & 720 & M & & & & \\
\hline Guinea Marsh & $11 / 9 / 1999$ & Transparent & New & 720 & M & & & & \\
\hline Guinea Marsh & $11 / 9 / 1999$ & Transparent & New & 704 & M & & & & \\
\hline Guinea Marsh & $11 / 9 / 1999$ & Transparent & New & 736 & M & & & & \\
\hline Guinea Marsh & $11 / 9 / 1999$ & Transparent & New & 700 & M & & & & \\
\hline Guinea Marsh & $11 / 9 / 1999$ & Transparent & New & 716 & M & & & & \\
\hline Guinea Marsh & $11 / 9 / 1999$ & Transparent & New & 720 & M & & & & \\
\hline Guinea Marsh & 11/9/1999 & Transparent & New & 706 & M & & & & \\
\hline Guinea Marsh & $11 / 9 / 1999$ & Transparent & New & 700 & M & & & & \\
\hline Guinea Marsh & $11 / 9 / 1999$ & Transparent & New & 700 & M & & & & \\
\hline Guinea Marsh & 11/9/1999 & Transparent & New & 684 & M & & & & \\
\hline Guinea Marsh & $11 / 9 / 1999$ & Transparent & New & 720 & M & & & & \\
\hline Guinea Marsh & $11 / 9 / 1999$ & Transparent & New & 708 & M & & & & \\
\hline
\end{tabular}


Appendix A (continued)

\begin{tabular}{|c|c|c|c|c|c|c|c|c|c|}
\hline Site & Date & Color & Age & $\begin{array}{l}\text { Size } \\
(\mu \mathrm{m})\end{array}$ & Sex & $\begin{array}{l}\text { Mean size } \\
\text { Guinea }(\mu \mathrm{m})\end{array}$ & $\begin{array}{l}\text { Mean size } \\
\text { Goodwin }(\mu \mathrm{m})\end{array}$ & $\begin{array}{l}\text { Mean size } \\
\text { Dameron }(\mu \mathrm{m})\end{array}$ & $\begin{array}{l}\text { Grand mean } \\
(\mu \mathrm{m})\end{array}$ \\
\hline Guinea Marsh & $11 / 9 / 1999$ & Transparent & New & 720 & M & & & & \\
\hline Guinea Marsh & 11/9/1999 & Transparent & New & 706 & M & & & & \\
\hline Guinea Marsh & $11 / 9 / 1999$ & Transparent & New & 716 & M & & & & \\
\hline Guinea Marsh & $11 / 9 / 1999$ & Transparent & New & 720 & M & & & & \\
\hline Guinea Marsh & 11/9/1999 & Transparent & New & 708 & M & & & & \\
\hline Guinea Marsh & $11 / 9 / 1999$ & Transparent & New & 700 & M & & & & \\
\hline Guinea Marsh & $11 / 9 / 1999$ & Transparent & New & 696 & M & & & & \\
\hline Guinea Marsh & $11 / 9 / 1999$ & Transparent & New & 714 & M & & & & \\
\hline Guinea Marsh & $11 / 9 / 1999$ & Transparent & New & 700 & M & & & & \\
\hline Guinea Marsh & $11 / 9 / 1999$ & Transparent & New & 718 & M & & & & \\
\hline Guinea Marsh & 11/9/1999 & Transparent & New & 724 & M & & & & \\
\hline Guinea Marsh & $11 / 9 / 1999$ & Transparent & New & 690 & M & & & & \\
\hline Guinea Marsh & $11 / 9 / 1999$ & Transparent & New & 736 & M & & & & \\
\hline Guinea Marsh & 11/9/1999 & Transparent & New & 730 & M & & & & \\
\hline Guinea Marsh & $11 / 9 / 1999$ & Transparent & New & 720 & M & & & & \\
\hline Guinea Marsh & 11/9/1999 & Transparent & New & 716 & M & & & & \\
\hline Guinea Marsh & 11/9/1999 & Transparent & New & 696 & M & & & & \\
\hline Guinea Marsh & $11 / 9 / 1999$ & Brown & Old & 710 & M & & & & \\
\hline Guinea Marsh & 11/9/1999 & Brown & Old & 704 & M & & & & \\
\hline Guinea Marsh & $11 / 9 / 1999$ & Brown & Old & 724 & M & & & & \\
\hline Guinea Marsh & $11 / 9 / 1999$ & Brown & Old & 720 & M & & & & \\
\hline Guinea Marsh & 11/9/1999 & Brown & Old & 704 & M & & & & \\
\hline Guinea Marsh & $11 / 9 / 1999$ & Brown-white & Old & 714 & M & & & & \\
\hline Guinea Marsh & 11/9/1999 & Brown-white & Old & 740 & M & & & & \\
\hline Goodwin Island & $12 / 21 / 1999$ & Trans-brown & Intermediate & 718 & M & & 703.4117647 & & 702.7894737 \\
\hline Goodwin Island & $12 / 21 / 1999$ & Trans-brown & Intermediate & 704 & M & & & & \\
\hline Goodwin Island & $12 / 21 / 1999$ & Trans-brown & Intermediate & 700 & M & & & & \\
\hline Goodwin Island & $12 / 21 / 1999$ & Trans-brown & Intermediate & 720 & M & & & & \\
\hline Goodwin Island & $12 / 21 / 1999$ & Trans-brown & Intermediate & 700 & M & & & & \\
\hline Goodwin Island & $12 / 21 / 1999$ & Trans-brown & Intermediate & 710 & M & & & & \\
\hline Goodwin Island & $12 / 21 / 1999$ & Transparent & New & 714 & M & & & & \\
\hline Goodwin Island & $12 / 21 / 1999$ & Transparent & New & 684 & M & & & & \\
\hline Goodwin Island & $12 / 21 / 1999$ & Transparent & New & 700 & M & & & & \\
\hline Goodwin Island & $12 / 21 / 1999$ & Brown & Old & 724 & M & & & & \\
\hline Goodwin Island & $12 / 21 / 1999$ & Brown-white & Old & 704 & M & & & & \\
\hline Goodwin Island & $12 / 21 / 1999$ & Very brown & Very old & 700 & M & & & & \\
\hline Goodwin Island & $12 / 21 / 1999$ & Very brown & Very old & 712 & M & & & & \\
\hline Goodwin Island & $12 / 21 / 1999$ & White-opaque & Very old & 700 & M & & & & \\
\hline Goodwin Island & $12 / 21 / 1999$ & White-opaque & Very old & 704 & M & & & & \\
\hline Goodwin Island & $12 / 21 / 1999$ & White-opaque & Very old & 684 & M & & & & \\
\hline Goodwin Island & $12 / 21 / 1999$ & White-opaque & Very old & 680 & M & & & & \\
\hline Guinea Marsh & $12 / 21 / 1999$ & Trans-white & Intermediate/old & 718 & M & 702.2857143 & & & \\
\hline Guinea Marsh & $12 / 21 / 1999$ & Trans-white & Intermediate/old & 718 & M & & & & \\
\hline Guinea Marsh & $12 / 21 / 1999$ & Trans-white & Intermediate/old & 724 & M & & & & \\
\hline Guinea Marsh & $12 / 21 / 1999$ & Trans-white & Intermediate/old & 700 & M & & & & \\
\hline Guinea Marsh & $12 / 21 / 1999$ & Transparent & New & 700 & M & & & & \\
\hline Guinea Marsh & $12 / 21 / 1999$ & Transparent & New & 714 & M & & & & \\
\hline Guinea Marsh & $12 / 21 / 1999$ & Transparent & New & 712 & M & & & & \\
\hline Guinea Marsh & $12 / 21 / 1999$ & Transparent & New & 700 & M & & & & \\
\hline Guinea Marsh & $12 / 21 / 1999$ & Transparent & New & 690 & M & & & & \\
\hline Guinea Marsh & $12 / 21 / 1999$ & Transparent & New & 700 & M & & & & \\
\hline Guinea Marsh & $12 / 21 / 1999$ & White-opaque & Very old & 680 & M & & & & \\
\hline Guinea Marsh & $12 / 21 / 1999$ & White-opaque & Very old & 688 & M & & & & \\
\hline
\end{tabular}


Appendix A (continued)

\begin{tabular}{|c|c|c|c|c|c|c|c|c|c|}
\hline Site & Date & Color & Age & $\begin{array}{l}\text { Size } \\
(\mu \mathrm{m})\end{array}$ & Sex & $\begin{array}{l}\text { Mean size } \\
\text { Guinea }(\mu \mathrm{m})\end{array}$ & $\begin{array}{l}\text { Mean size } \\
\text { Goodwin }(\mu \mathrm{m})\end{array}$ & $\begin{array}{l}\text { Mean size } \\
\text { Dameron }(\mu \mathrm{m})\end{array}$ & $\begin{array}{l}\text { Grand mean } \\
(\mu \mathrm{m})\end{array}$ \\
\hline Guinea Marsh & $12 / 21 / 1999$ & White-opaque & Very old & 720 & M & & & & \\
\hline Guinea Marsh & $12 / 21 / 1999$ & White-opaque & Very old & 724 & M & & & & \\
\hline Guinea Marsh & $12 / 21 / 1999$ & White-opaque & Very old & 704 & M & & & & \\
\hline Guinea Marsh & $12 / 21 / 1999$ & White-opaque & Very old & 720 & M & & & & \\
\hline Guinea Marsh & $12 / 21 / 1999$ & White-opaque & Very old & 698 & M & & & & \\
\hline Guinea Marsh & $12 / 21 / 1999$ & White-opaque & Very old & 704 & M & & & & \\
\hline Guinea Marsh & $12 / 21 / 1999$ & White-opaque & Very old & 710 & M & & & & \\
\hline Guinea Marsh & $12 / 21 / 1999$ & White-opaque & Very old & 620 & M & & & & \\
\hline Guinea Marsh & $12 / 21 / 1999$ & White-opaque & Very old & 704 & M & & & & \\
\hline Goodwin Island & $1 / 8 / 2000$ & Trans-brown & Intermediate & 700 & M & & 710.4444444 & & 707.3571429 \\
\hline Goodwin Island & $1 / 8 / 2000$ & Trans-brown & Intermediate & 710 & M & & & & \\
\hline Goodwin Island & $1 / 8 / 2000$ & Transparent & New & 712 & M & & & & \\
\hline Goodwin Island & $1 / 8 / 2000$ & Transparent & New & 704 & M & & & & \\
\hline Goodwin Island & $1 / 8 / 2000$ & Transparent & New & 706 & M & & & & \\
\hline Goodwin Island & $1 / 8 / 2000$ & Brown & Old & 720 & M & & & & \\
\hline Goodwin Island & $1 / 8 / 2000$ & Brown & Old & 720 & M & & & & \\
\hline Goodwin Island & $1 / 8 / 2000$ & Brown-white & Old & 720 & M & & & & \\
\hline Goodwin Island & $1 / 8 / 2000$ & Very brown & Very old & 702 & M & & & & \\
\hline Guinea Marsh & $1 / 8 / 2000$ & Trans-brown & Intermediate & 718 & M & 705.8947368 & & & \\
\hline Guinea Marsh & $1 / 8 / 2000$ & Trans-brown & Intermediate & 702 & M & & & & \\
\hline Guinea Marsh & $1 / 8 / 2000$ & Trans-brown & Intermediate & 706 & M & & & & \\
\hline Guinea Marsh & $1 / 8 / 2000$ & Transparent & New & 710 & M & & & & \\
\hline Guinea Marsh & $1 / 8 / 2000$ & Transparent & New & 720 & M & & & & \\
\hline Guinea Marsh & $1 / 8 / 2000$ & Transparent & New & 664 & M & & & & \\
\hline Guinea Marsh & $1 / 8 / 2000$ & Transparent & New & 700 & M & & & & \\
\hline Guinea Marsh & $1 / 8 / 2000$ & Transparent & New & 700 & M & & & & \\
\hline Guinea Marsh & $1 / 8 / 2000$ & Transparent & New & 700 & M & & & & \\
\hline Guinea Marsh & $1 / 8 / 2000$ & Brown & Old & 720 & M & & & & \\
\hline Guinea Marsh & $1 / 8 / 2000$ & Brown & Old & 710 & M & & & & \\
\hline Guinea Marsh & $1 / 8 / 2000$ & Brown & Old & 700 & M & & & & \\
\hline Guinea Marsh & $1 / 8 / 2000$ & Brown & Old & 730 & M & & & & \\
\hline Guinea Marsh & $1 / 8 / 2000$ & Brown & Old & 694 & M & & & & \\
\hline Guinea Marsh & $1 / 8 / 2000$ & Brown & Old & 704 & M & & & & \\
\hline Guinea Marsh & $1 / 8 / 2000$ & Brown & Old & 710 & M & & & & \\
\hline Guinea Marsh & $1 / 8 / 2000$ & Brown & Old & 720 & M & & & & \\
\hline Guinea Marsh & $1 / 8 / 2000$ & Brown-white & Old & 704 & M & & & & \\
\hline Guinea Marsh & $1 / 8 / 2000$ & Very brown & Very old & 700 & M & & & & \\
\hline Guinea Marsh & $2 / 29 / 2000$ & Trans-brown & Intermediate & 688 & M & 708.36 & & & 708.36 \\
\hline Guinea Marsh & $2 / 29 / 2000$ & Trans-brown & Intermediate & 716 & M & & & & \\
\hline Guinea Marsh & $2 / 29 / 2000$ & Trans-brown & Intermediate & 702 & M & & & & \\
\hline Guinea Marsh & $2 / 29 / 2000$ & Trans-brown & Intermediate & 706 & M & & & & \\
\hline Guinea Marsh & $2 / 29 / 2000$ & Trans-brown & Intermediate & 700 & M & & & & \\
\hline Guinea Marsh & $2 / 29 / 2000$ & Trans-brown & Intermediate & 708 & M & & & & \\
\hline Guinea Marsh & $2 / 29 / 2000$ & Trans-brown & Intermediate & 706 & M & & & & \\
\hline Guinea Marsh & $2 / 29 / 2000$ & Trans-brown & Intermediate & 716 & M & & & & \\
\hline Guinea Marsh & $2 / 29 / 2000$ & Trans-brown & Intermediate & 724 & M & & & & \\
\hline Guinea Marsh & $2 / 29 / 2000$ & Trans-brown & Intermediate & 720 & M & & & & \\
\hline Guinea Marsh & $2 / 29 / 2000$ & Trans-brown & Intermediate & 718 & M & & & & \\
\hline Guinea Marsh & $2 / 29 / 2000$ & Trans-brown & Intermediate & 712 & M & & & & \\
\hline Guinea Marsh & $2 / 29 / 2000$ & Trans-brown & Intermediate & 720 & M & & & & \\
\hline Guinea Marsh & $2 / 29 / 2000$ & Trans-brown & Intermediate & 720 & M & & & & \\
\hline Guinea Marsh & $2 / 29 / 2000$ & Transparent & New & 690 & M & & & & \\
\hline
\end{tabular}


Appendix A (continued)

\begin{tabular}{|c|c|c|c|c|c|c|c|c|c|}
\hline Site & Date & Color & Age & $\begin{array}{l}\text { Size } \\
(\mu \mathrm{m})\end{array}$ & Sex & $\begin{array}{l}\text { Mean size } \\
\text { Guinea }(\mu \mathrm{m})\end{array}$ & $\begin{array}{l}\text { Mean size } \\
\text { Goodwin }(\mu \mathrm{m})\end{array}$ & $\begin{array}{l}\text { Mean size } \\
\text { Dameron }(\mu \mathrm{m})\end{array}$ & $\begin{array}{l}\text { Grand mean } \\
(\mu \mathrm{m})\end{array}$ \\
\hline Guinea Marsh & $2 / 29 / 2000$ & Transparent & New & 724 & M & & & & \\
\hline Guinea Marsh & $2 / 29 / 2000$ & Transparent & New & 722 & M & & & & \\
\hline Guinea Marsh & $2 / 29 / 2000$ & Transparent & New & 724 & M & & & & \\
\hline Guinea Marsh & $2 / 29 / 2000$ & Transparent & New & 700 & M & & & & \\
\hline Guinea Marsh & $2 / 29 / 2000$ & Brown & Old & 700 & M & & & & \\
\hline Guinea Marsh & $2 / 29 / 2000$ & Brown & Old & 704 & M & & & & \\
\hline Guinea Marsh & $2 / 29 / 2000$ & Brown & Old & 700 & M & & & & \\
\hline Guinea Marsh & $2 / 29 / 2000$ & Brown & Old & 710 & M & & & & \\
\hline Guinea Marsh & $2 / 29 / 2000$ & Brown & Old & 708 & M & & & & \\
\hline Guinea Marsh & $2 / 29 / 2000$ & Brown & Old & 720 & M & & & & \\
\hline Guinea Marsh & $2 / 29 / 2000$ & Brown & Old & 704 & M & & & & \\
\hline Guinea Marsh & $2 / 29 / 2000$ & Brown & Old & 700 & M & & & & \\
\hline Guinea Marsh & $2 / 29 / 2000$ & Brown & Old & 690 & M & & & & \\
\hline Guinea Marsh & $2 / 29 / 2000$ & Brown & Old & 720 & M & & & & \\
\hline Guinea Marsh & $2 / 29 / 2000$ & Brown & Old & 700 & M & & & & \\
\hline Guinea Marsh & $2 / 29 / 2000$ & Brown & Old & 696 & M & & & & \\
\hline Guinea Marsh & $2 / 29 / 2000$ & Brown & Old & 720 & M & & & & \\
\hline Guinea Marsh & $2 / 29 / 2000$ & Brown & Old & 702 & M & & & & \\
\hline Guinea Marsh & $2 / 29 / 2000$ & Brown & Old & 714 & M & & & & \\
\hline Guinea Marsh & $2 / 29 / 2000$ & Brown & Old & 718 & M & & & & \\
\hline Guinea Marsh & $2 / 29 / 2000$ & Brown & Old & 694 & M & & & & \\
\hline Guinea Marsh & $2 / 29 / 2000$ & Brown & Old & 700 & M & & & & \\
\hline Guinea Marsh & $2 / 29 / 2000$ & Brown & Old & 722 & M & & & & \\
\hline Guinea Marsh & $2 / 29 / 2000$ & Brown & Old & 684 & M & & & & \\
\hline Guinea Marsh & $2 / 29 / 2000$ & Brown & Old & 710 & M & & & & \\
\hline Guinea Marsh & $2 / 29 / 2000$ & Brown & Old & 690 & M & & & & \\
\hline Guinea Marsh & $2 / 29 / 2000$ & Brown & Old & 726 & M & & & & \\
\hline Guinea Marsh & $2 / 29 / 2000$ & Brown & Old & 700 & M & & & & \\
\hline Guinea Marsh & $2 / 29 / 2000$ & Brown & Old & 720 & M & & & & \\
\hline Guinea Marsh & $2 / 29 / 2000$ & Brown & Old & 700 & M & & & & \\
\hline Guinea Marsh & $2 / 29 / 2000$ & Brown-white & Old & 706 & M & & & & \\
\hline Guinea Marsh & $2 / 29 / 2000$ & Brown-white & Old & 718 & M & & & & \\
\hline Guinea Marsh & $2 / 29 / 2000$ & Brown-white & Old & 700 & M & & & & \\
\hline Guinea Marsh & $2 / 29 / 2000$ & Very brown & Very old & 724 & M & & & & \\
\hline Guinea Marsh & $2 / 29 / 2000$ & White-opaque & Very old & 702 & M & & & & \\
\hline Guinea Marsh & $3 / 29 / 2000$ & Trans-white & Intermediate/old & 686 & M & 704 & & & 704 \\
\hline Guinea Marsh & $3 / 29 / 2000$ & Trans-white & Intermediate/old & 700 & M & & & & \\
\hline Guinea Marsh & $3 / 29 / 2000$ & Brown-white & Old & 722 & M & & & & \\
\hline Guinea Marsh & $3 / 29 / 2000$ & Brown-white & Old & 700 & M & & & & \\
\hline Guinea Marsh & $3 / 29 / 2000$ & Brown-white & Old & 724 & M & & & & \\
\hline Guinea Marsh & $3 / 29 / 2000$ & White-opaque & Very old & 698 & M & & & & \\
\hline Guinea Marsh & $3 / 29 / 2000$ & White-opaque & Very old & 704 & M & & & & \\
\hline Guinea Marsh & $3 / 29 / 2000$ & White-opaque & Very old & 698 & M & & & & \\
\hline Goodwin Island & $4 / 19 / 2000$ & Brown-white & Old & 704 & M & & 694 & & 694 \\
\hline Goodwin Island & $4 / 19 / 2000$ & Brown-white & Old & 684 & M & & & & \\
\hline Dameron Marsh & $5 / 17 / 2000$ & Transparent & New & 700 & M & & & 701 & 701 \\
\hline Dameron Marsh & $5 / 17 / 2000$ & Transparent & New & 702 & M & & & & \\
\hline Goodwin Island & $6 / 13 / 2000$ & Trans-brown & Intermediate & 708 & M & & 710.7368421 & & 711 \\
\hline Goodwin Island & $6 / 13 / 2000$ & Trans-brown & Intermediate & 716 & M & & & & \\
\hline Goodwin Island & $6 / 13 / 2000$ & Trans-white & Intermediate/old & 702 & M & & & & \\
\hline Goodwin Island & $6 / 13 / 2000$ & Transparent & New & 730 & M & & & & \\
\hline Goodwin Island & $6 / 13 / 2000$ & Transparent & New & 700 & M & & & & \\
\hline Goodwin Island & $6 / 13 / 2000$ & Transparent & New & 700 & M & & & & \\
\hline
\end{tabular}


Appendix A (continued)

\begin{tabular}{|c|c|c|c|c|c|c|c|c|c|}
\hline Site & Date & Color & Age & $\begin{array}{l}\text { Size } \\
(\mu \mathrm{m})\end{array}$ & Sex & $\begin{array}{l}\text { Mean size } \\
\text { Guinea }(\mu \mathrm{m})\end{array}$ & $\begin{array}{l}\text { Mean size } \\
\text { Goodwin }(\mu \mathrm{m})\end{array}$ & $\begin{array}{l}\text { Mean size } \\
\text { Dameron }(\mu \mathrm{m})\end{array}$ & $\begin{array}{l}\text { Grand mean } \\
(\mu \mathrm{m})\end{array}$ \\
\hline Goodwin Island & $6 / 13 / 2000$ & Transparent & New & 730 & M & & & & \\
\hline Goodwin Island & $6 / 13 / 2000$ & Transparent & New & 702 & M & & & & \\
\hline Goodwin Island & $6 / 13 / 2000$ & Transparent & New & 720 & M & & & & \\
\hline Goodwin Island & $6 / 13 / 2000$ & Transparent & New & 720 & M & & & & \\
\hline Goodwin Island & $6 / 13 / 2000$ & Transparent & New & 704 & M & & & & \\
\hline Goodwin Island & $6 / 13 / 2000$ & Transparent & New & 704 & M & & & & \\
\hline Goodwin Island & $6 / 13 / 2000$ & Transparent & New & 690 & M & & & & \\
\hline Goodwin Island & $6 / 13 / 2000$ & Transparent & New & 720 & M & & & & \\
\hline Goodwin Island & $6 / 13 / 2000$ & Transparent & New & 700 & M & & & & \\
\hline Goodwin Island & $6 / 13 / 2000$ & Transparent & New & 724 & M & & & & \\
\hline Goodwin Island & $6 / 13 / 2000$ & Transparent & New & 720 & M & & & & \\
\hline Goodwin Island & $6 / 13 / 2000$ & Brown & Old & 690 & M & & & & \\
\hline Goodwin Island & $6 / 13 / 2000$ & Brown & Old & 724 & M & & & & \\
\hline Guinea Marsh & $6 / 13 / 2000$ & Trans-brown & Intermediate & 720 & M & 712.6666667 & & & \\
\hline Guinea Marsh & $6 / 13 / 2000$ & Trans-white & Intermediate/old & 714 & M & & & & \\
\hline Guinea Marsh & $6 / 13 / 2000$ & Transparent & New & 704 & M & & & & \\
\hline Goodwin Island & $7 / 11 / 2000$ & Trans-brown & Intermediate & 720 & M & & 713.1428571 & & 714.7692308 \\
\hline Goodwin Island & $7 / 11 / 2000$ & Transparent & New & 718 & M & & & & \\
\hline Goodwin Island & $7 / 11 / 2000$ & Transparent & New & 700 & M & & & & \\
\hline Goodwin Island & $7 / 11 / 2000$ & Transparent & New & 720 & M & & & & \\
\hline Goodwin Island & $7 / 11 / 2000$ & Transparent & New & 718 & M & & & & \\
\hline Goodwin Island & $7 / 11 / 2000$ & Brown & Old & 700 & M & & & & \\
\hline Goodwin Island & $7 / 11 / 2000$ & Brown & Old & 740 & M & & & & \\
\hline Goodwin Island & $7 / 11 / 2000$ & Brown & Old & 700 & M & & & & \\
\hline Goodwin Island & $7 / 11 / 2000$ & Brown & Old & 710 & M & & & & \\
\hline Goodwin Island & $7 / 11 / 2000$ & Brown & Old & 680 & M & & & & \\
\hline Goodwin Island & $7 / 11 / 2000$ & Brown & Old & 720 & M & & & & \\
\hline Goodwin Island & $7 / 11 / 2000$ & Brown-white & Old & 730 & M & & & & \\
\hline Goodwin Island & $7 / 11 / 2000$ & Brown-white & Old & 710 & M & & & & \\
\hline Goodwin Island & $7 / 11 / 2000$ & White-opaque & Very old & 718 & M & & & & \\
\hline Guinea Marsh & $7 / 11 / 2000$ & Trans-white & Intermediate/old & 740 & M & 716.6666667 & & & \\
\hline Guinea Marsh & $7 / 11 / 2000$ & Trans-white & Intermediate/old & 706 & M & & & & \\
\hline Guinea Marsh & $7 / 11 / 2000$ & Trans-white & Intermediate/old & 716 & M & & & & \\
\hline Guinea Marsh & $7 / 11 / 2000$ & Brown & Old & 720 & M & & & & \\
\hline Guinea Marsh & $7 / 11 / 2000$ & Brown-white & Old & 740 & M & & & & \\
\hline Guinea Marsh & $7 / 11 / 2000$ & Brown-white & Old & 700 & M & & & & \\
\hline Guinea Marsh & $7 / 11 / 2000$ & Brown-white & Old & 740 & M & & & & \\
\hline Guinea Marsh & $7 / 11 / 2000$ & Brown-white & Old & 702 & M & & & & \\
\hline Guinea Marsh & $7 / 11 / 2000$ & Very brown & Very old & 704 & M & & & & \\
\hline Guinea Marsh & $7 / 11 / 2000$ & White-opaque & Very old & 718 & M & & & & \\
\hline Guinea Marsh & $7 / 11 / 2000$ & White-opaque & Very old & 704 & M & & & & \\
\hline Guinea Marsh & $7 / 11 / 2000$ & White-opaque & Very old & 710 & M & & & & \\
\hline Goodwin Island & $8 / 1 / 2000$ & White-opaque & Very old & 720 & M & & 712 & & 715.1666667 \\
\hline Goodwin Island & $8 / 1 / 2000$ & White-opaque & Very old & 704 & M & & & & \\
\hline Guinea Marsh & $8 / 1 / 2000$ & Trans-brown & Intermediate & 700 & M & 715.8 & & & \\
\hline Guinea Marsh & $8 / 1 / 2000$ & Trans-white & Intermediate/old & 724 & M & & & & \\
\hline Guinea Marsh & $8 / 1 / 2000$ & Transparent & New & 724 & M & & & & \\
\hline Guinea Marsh & $8 / 1 / 2000$ & Brown & Old & 700 & M & & & & \\
\hline Guinea Marsh & $8 / 1 / 2000$ & Brown & Old & 730 & M & & & & \\
\hline Guinea Marsh & $8 / 1 / 2000$ & Brown & Old & 714 & M & & & & \\
\hline Guinea Marsh & $8 / 1 / 2000$ & Brown-white & Old & 700 & M & & & & \\
\hline Guinea Marsh & $8 / 1 / 2000$ & Brown-white & Old & 708 & M & & & & \\
\hline
\end{tabular}


Appendix A (continued)

\begin{tabular}{|c|c|c|c|c|c|c|c|c|c|}
\hline Site & Date & Color & Age & $\begin{array}{l}\text { Size } \\
(\mu \mathrm{m})\end{array}$ & Sex & $\begin{array}{l}\text { Mean size } \\
\text { Guinea }(\mu \mathrm{m})\end{array}$ & $\begin{array}{l}\text { Mean size } \\
\text { Goodwin }(\mu \mathrm{m})\end{array}$ & $\begin{array}{l}\text { Mean size } \\
\text { Dameron }(\mu \mathrm{m})\end{array}$ & $\begin{array}{l}\text { Grand mean } \\
(\mu \mathrm{m})\end{array}$ \\
\hline Guinea Marsh & $8 / 1 / 2000$ & Brown-white & Old & 720 & M & & & & \\
\hline Guinea Marsh & $8 / 1 / 2000$ & Brown-white & Old & 738 & M & & & & \\
\hline Goodwin Island & $8 / 22 / 2000$ & Trans-brown & Intermediate & 724 & M & & 707.5 & & 703.2307692 \\
\hline Goodwin Island & $8 / 22 / 2000$ & Trans-brown & Intermediate & 704 & M & & & & \\
\hline Goodwin Island & $8 / 22 / 2000$ & Trans-brown & Intermediate & 702 & M & & & & \\
\hline Goodwin Island & $8 / 22 / 2000$ & Trans-brown & Intermediate & 680 & M & & & & \\
\hline Goodwin Island & $8 / 22 / 2000$ & Trans-brown & Intermediate & 718 & M & & & & \\
\hline Goodwin Island & $8 / 22 / 2000$ & Trans-brown & Intermediate & 700 & M & & & & \\
\hline Goodwin Island & $8 / 22 / 2000$ & Trans-brown & Intermediate & 680 & M & & & & \\
\hline Goodwin Island & $8 / 22 / 2000$ & Trans-white & Intermediate/old & 720 & M & & & & \\
\hline Goodwin Island & $8 / 22 / 2000$ & Trans-white & Intermediate/old & 710 & M & & & & \\
\hline Goodwin Island & $8 / 22 / 2000$ & Trans-white & Intermediate/old & 704 & M & & & & \\
\hline Goodwin Island & $8 / 22 / 2000$ & Transparent & New & 704 & M & & & & \\
\hline Goodwin Island & $8 / 22 / 2000$ & Transparent & New & 684 & M & & & & \\
\hline Goodwin Island & $8 / 22 / 2000$ & Transparent & New & 688 & M & & & & \\
\hline Goodwin Island & $8 / 22 / 2000$ & Transparent & New & 700 & M & & & & \\
\hline Goodwin Island & $8 / 22 / 2000$ & Transparent & New & 720 & M & & & & \\
\hline Goodwin Island & $8 / 22 / 2000$ & Transparent & New & 720 & M & & & & \\
\hline Goodwin Island & $8 / 22 / 2000$ & Transparent & New & 710 & M & & & & \\
\hline Goodwin Island & $8 / 22 / 2000$ & Transparent & New & 724 & M & & & & \\
\hline Goodwin Island & $8 / 22 / 2000$ & Transparent & New & 704 & M & & & & \\
\hline Goodwin Island & $8 / 22 / 2000$ & Brown & Old & 740 & M & & & & \\
\hline Goodwin Island & $8 / 22 / 2000$ & Brown & Old & 722 & M & & & & \\
\hline Goodwin Island & $8 / 22 / 2000$ & Brown & Old & 704 & M & & & & \\
\hline Goodwin Island & $8 / 22 / 2000$ & Brown-white & Old & 722 & M & & & & \\
\hline Goodwin Island & $8 / 22 / 2000$ & Brown-white & Old & 722 & M & & & & \\
\hline Goodwin Island & $8 / 22 / 2000$ & Brown-white & Old & 710 & M & & & & \\
\hline Goodwin Island & $8 / 22 / 2000$ & Brown-white & Old & 706 & M & & & & \\
\hline Goodwin Island & $8 / 22 / 2000$ & White-opaque & Very old & 724 & M & & & & \\
\hline Goodwin Island & $8 / 22 / 2000$ & White-opaque & Very old & 664 & M & & & & \\
\hline Guinea Marsh & $8 / 22 / 2000$ & Trans-brown & Intermediate & 700 & M & 698.25 & & & \\
\hline Guinea Marsh & $8 / 22 / 2000$ & Trans-brown & Intermediate & 700 & M & & & & \\
\hline Guinea Marsh & $8 / 22 / 2000$ & Trans-white & Intermediate/old & 680 & M & & & & \\
\hline Guinea Marsh & $8 / 22 / 2000$ & Trans-white & Intermediate/old & 680 & M & & & & \\
\hline Guinea Marsh & $8 / 22 / 2000$ & Transparent & New & 680 & M & & & & \\
\hline Guinea Marsh & $8 / 22 / 2000$ & Transparent & New & 704 & M & & & & \\
\hline Guinea Marsh & $8 / 22 / 2000$ & Transparent & New & 720 & M & & & & \\
\hline Guinea Marsh & $8 / 22 / 2000$ & Transparent & New & 690 & M & & & & \\
\hline Guinea Marsh & $8 / 22 / 2000$ & Transparent & New & 710 & M & & & & \\
\hline Guinea Marsh & $8 / 22 / 2000$ & Transparent & New & 684 & M & & & & \\
\hline Guinea Marsh & $8 / 22 / 2000$ & Transparent & New & 716 & M & & & & \\
\hline Guinea Marsh & $8 / 22 / 2000$ & Transparent & New & 700 & M & & & & \\
\hline Guinea Marsh & $8 / 22 / 2000$ & Transparent & New & 700 & M & & & & \\
\hline Guinea Marsh & $8 / 22 / 2000$ & Transparent & New & 704 & M & & & & \\
\hline Guinea Marsh & $8 / 22 / 2000$ & Transparent & New & 700 & M & & & & \\
\hline Guinea Marsh & $8 / 22 / 2000$ & Transparent & New & 700 & M & & & & \\
\hline Guinea Marsh & $8 / 22 / 2000$ & Transparent & New & 680 & M & & & & \\
\hline Guinea Marsh & $8 / 22 / 2000$ & Transparent & New & 684 & M & & & & \\
\hline Guinea Marsh & $8 / 22 / 2000$ & Transparent & New & 704 & M & & & & \\
\hline Guinea Marsh & $8 / 22 / 2000$ & Transparent & New & 706 & M & & & & \\
\hline Guinea Marsh & $8 / 22 / 2000$ & Transparent & New & 710 & M & & & & \\
\hline Guinea Marsh & $8 / 22 / 2000$ & Transparent & New & 706 & M & & & & \\
\hline Guinea Marsh & $8 / 22 / 2000$ & Brown & Old & 700 & M & & & & \\
\hline
\end{tabular}


Appendix A (continued)

\begin{tabular}{|c|c|c|c|c|c|c|c|c|c|}
\hline Site & Date & Color & Age & $\begin{array}{l}\text { Size } \\
(\mu \mathrm{m})\end{array}$ & Sex & $\begin{array}{l}\text { Mean size } \\
\text { Guinea }(\mu \mathrm{m})\end{array}$ & $\begin{array}{l}\text { Mean size } \\
\text { Goodwin }(\mu \mathrm{m})\end{array}$ & $\begin{array}{l}\text { Mean size } \\
\text { Dameron }(\mu \mathrm{m})\end{array}$ & $\begin{array}{l}\text { Grand mean } \\
(\mu \mathrm{m})\end{array}$ \\
\hline Guinea Marsh & $8 / 22 / 2000$ & Very brown & Very old & 700 & M & & & & \\
\hline Goodwin Island & $9 / 6 / 2000$ & Trans-white & Intermediate/old & 700 & M & & 705.6 & & 713.8666667 \\
\hline Goodwin Island & $9 / 6 / 2000$ & Transparent & New & 690 & M & & & & \\
\hline Goodwin Island & $9 / 6 / 2000$ & Brown-white & Old & 710 & M & & & & \\
\hline Goodwin Island & $9 / 6 / 2000$ & Brown-white & Old & 720 & M & & & & \\
\hline Goodwin Island & $9 / 6 / 2000$ & Brown-white & Old & 708 & M & & & & \\
\hline Guinea Marsh & $9 / 6 / 2000$ & Trans-white & Intermediate/old & 720 & M & 718 & & & \\
\hline Guinea Marsh & $9 / 6 / 2000$ & Trans-white & Intermediate/old & 700 & M & & & & \\
\hline Guinea Marsh & $9 / 6 / 2000$ & Trans-white & Intermediate/old & 724 & M & & & & \\
\hline Guinea Marsh & $9 / 6 / 2000$ & Transparent & New & 720 & M & & & & \\
\hline Guinea Marsh & $9 / 6 / 2000$ & Brown & Old & 704 & M & & & & \\
\hline Guinea Marsh & $9 / 6 / 2000$ & Brown & Old & 730 & M & & & & \\
\hline Guinea Marsh & $9 / 6 / 2000$ & Brown & Old & 720 & M & & & & \\
\hline Guinea Marsh & $9 / 6 / 2000$ & Brown-white & Old & 712 & M & & & & \\
\hline Guinea Marsh & $9 / 6 / 2000$ & Brown-white & Old & 730 & M & & & & \\
\hline Guinea Marsh & $9 / 6 / 2000$ & Brown-white & Old & 720 & M & & & & \\
\hline Goodwin Island & $9 / 20 / 2000$ & Trans-brown & Intermediate & 684 & M & & 691.4285714 & & 699.68 \\
\hline Goodwin Island & $9 / 20 / 2000$ & Trans-brown & Intermediate & 720 & M & & & & \\
\hline Goodwin Island & $9 / 20 / 2000$ & Transparent & New & 700 & M & & & & \\
\hline Goodwin Island & $9 / 20 / 2000$ & Transparent & New & 660 & M & & & & \\
\hline Goodwin Island & $9 / 20 / 2000$ & Transparent & New & 700 & M & & & & \\
\hline Goodwin Island & $9 / 20 / 2000$ & Transparent & New & 696 & M & & & & \\
\hline Goodwin Island & $9 / 20 / 2000$ & Brown & Old & 680 & M & & & & \\
\hline Guinea Marsh & $9 / 20 / 2000$ & Trans-brown & Intermediate & 680 & M & 702.8888889 & & & \\
\hline Guinea Marsh & $9 / 20 / 2000$ & Trans-brown & Intermediate & 714 & M & & & & \\
\hline Guinea Marsh & $9 / 20 / 2000$ & Trans-brown & Intermediate & 710 & M & & & & \\
\hline Guinea Marsh & $9 / 20 / 2000$ & Trans-brown & Intermediate & 720 & M & & & & \\
\hline Guinea Marsh & $9 / 20 / 2000$ & Trans-white & Intermediate/old & 680 & M & & & & \\
\hline Guinea Marsh & $9 / 20 / 2000$ & Transparent & New & 690 & M & & & & \\
\hline Guinea Marsh & $9 / 20 / 2000$ & Transparent & New & 722 & M & & & & \\
\hline Guinea Marsh & $9 / 20 / 2000$ & Transparent & New & 720 & M & & & & \\
\hline Guinea Marsh & $9 / 20 / 2000$ & Transparent & New & 726 & M & & & & \\
\hline Guinea Marsh & $9 / 20 / 2000$ & Transparent & New & 698 & M & & & & \\
\hline Guinea Marsh & $9 / 20 / 2000$ & Transparent & New & 700 & M & & & & \\
\hline Guinea Marsh & $9 / 20 / 2000$ & Transparent & New & 704 & M & & & & \\
\hline Guinea Marsh & $9 / 20 / 2000$ & Transparent & New & 708 & M & & & & \\
\hline Guinea Marsh & $9 / 20 / 2000$ & Transparent & New & 700 & M & & & & \\
\hline Guinea Marsh & $9 / 20 / 2000$ & Brown & Old & 702 & M & & & & \\
\hline Guinea Marsh & $9 / 20 / 2000$ & Brown & Old & 680 & M & & & & \\
\hline Guinea Marsh & $9 / 20 / 2000$ & Brown & Old & 698 & M & & & & \\
\hline Guinea Marsh & $9 / 20 / 2000$ & White-opaque & Very old & 700 & M & & & & \\
\hline Goodwin Island & $10 / 3 / 2000$ & Transparent & New & 716 & M & & 701.7142857 & & 710.2962963 \\
\hline Goodwin Island & $10 / 3 / 2000$ & Transparent & New & 700 & M & & & & \\
\hline Goodwin Island & $10 / 3 / 2000$ & Transparent & New & 700 & M & & & & \\
\hline Goodwin Island & $10 / 3 / 2000$ & Transparent & New & 704 & M & & & & \\
\hline Goodwin Island & $10 / 3 / 2000$ & Transparent & New & 686 & M & & & & \\
\hline Goodwin Island & $10 / 3 / 2000$ & Transparent & New & 726 & M & & & & \\
\hline Goodwin Island & $10 / 3 / 2000$ & Brown & Old & 680 & M & & & & \\
\hline Guinea Marsh & $10 / 3 / 2000$ & Trans-brown & Intermediate & 700 & M & 713.3 & & & \\
\hline Guinea Marsh & $10 / 3 / 2000$ & Trans-white & Intermediate/old & 700 & M & & & & \\
\hline Guinea Marsh & $10 / 3 / 2000$ & Trans-white & Intermediate/old & 700 & M & & & & \\
\hline Guinea Marsh & $10 / 3 / 2000$ & Transparent & New & 718 & M & & & & \\
\hline Guinea Marsh & $10 / 3 / 2000$ & Transparent & New & 710 & M & & & & \\
\hline
\end{tabular}


Appendix A (continued)

\begin{tabular}{|c|c|c|c|c|c|c|c|c|c|}
\hline Site & Date & Color & Age & $\begin{array}{l}\text { Size } \\
(\mu \mathrm{m})\end{array}$ & Sex & $\begin{array}{l}\text { Mean size } \\
\text { Guinea }(\mu \mathrm{m})\end{array}$ & $\begin{array}{l}\text { Mean size } \\
\text { Goodwin }(\mu \mathrm{m})\end{array}$ & $\begin{array}{l}\text { Mean size } \\
\text { Dameron }(\mu \mathrm{m})\end{array}$ & $\begin{array}{l}\text { Grand mean } \\
(\mu \mathrm{m})\end{array}$ \\
\hline Guinea Marsh & $10 / 3 / 2000$ & Transparent & New & 740 & $\mathrm{M}$ & & & & \\
\hline Guinea Marsh & $10 / 3 / 2000$ & Transparent & New & 690 & M & & & & \\
\hline Guinea Marsh & $10 / 3 / 2000$ & Transparent & New & 730 & M & & & & \\
\hline Guinea Marsh & $10 / 3 / 2000$ & Transparent & New & 724 & M & & & & \\
\hline Guinea Marsh & $10 / 3 / 2000$ & Transparent & New & 720 & M & & & & \\
\hline Guinea Marsh & $10 / 3 / 2000$ & Brown & Old & 712 & M & & & & \\
\hline Guinea Marsh & $10 / 3 / 2000$ & Brown & Old & 724 & M & & & & \\
\hline Guinea Marsh & $10 / 3 / 2000$ & Brown & Old & 720 & M & & & & \\
\hline Guinea Marsh & $10 / 3 / 2000$ & Brown & Old & 712 & M & & & & \\
\hline Guinea Marsh & $10 / 3 / 2000$ & Brown & Old & 684 & M & & & & \\
\hline Guinea Marsh & $10 / 3 / 2000$ & Brown & Old & 714 & M & & & & \\
\hline Guinea Marsh & $10 / 3 / 2000$ & Brown & Old & 720 & M & & & & \\
\hline Guinea Marsh & $10 / 3 / 2000$ & Brown & Old & 728 & M & & & & \\
\hline Guinea Marsh & $10 / 3 / 2000$ & Very brown & Very old & 700 & M & & & & \\
\hline Guinea Marsh & $10 / 3 / 2000$ & White-opaque & Very old & 720 & M & & & & \\
\hline Goodwin Island & $11 / 14 / 2000$ & Trans-brown & Intermediate & 724 & M & & 707.030303 & & 708.8085106 \\
\hline Goodwin Island & $11 / 14 / 2000$ & Trans-brown & Intermediate & 720 & M & & & & \\
\hline Goodwin Island & $11 / 14 / 2000$ & Trans-brown & Intermediate & 698 & M & & & & \\
\hline Goodwin Island & $11 / 14 / 2000$ & Trans-brown & Intermediate & 702 & M & & & & \\
\hline Goodwin Island & $11 / 14 / 2000$ & Trans-brown & Intermediate & 722 & M & & & & \\
\hline Goodwin Island & $11 / 14 / 2000$ & Trans-brown & Intermediate & 724 & M & & & & \\
\hline Goodwin Island & $11 / 14 / 2000$ & Trans-brown & Intermediate & 718 & M & & & & \\
\hline Goodwin Island & $11 / 14 / 2000$ & Trans-brown & Intermediate & 720 & M & & & & \\
\hline Goodwin Island & $11 / 14 / 2000$ & Trans-brown & Intermediate & 680 & M & & & & \\
\hline Goodwin Island & $11 / 14 / 2000$ & Trans-white & Intermediate/old & 720 & M & & & & \\
\hline Goodwin Island & $11 / 14 / 2000$ & Trans-white & Intermediate/old & 700 & M & & & & \\
\hline Goodwin Island & $11 / 14 / 2000$ & Transparent & New & 692 & M & & & & \\
\hline Goodwin Island & $11 / 14 / 2000$ & Transparent & New & 698 & M & & & & \\
\hline Goodwin Island & $11 / 14 / 2000$ & Transparent & New & 690 & M & & & & \\
\hline Goodwin Island & $11 / 14 / 2000$ & Transparent & New & 700 & M & & & & \\
\hline Goodwin Island & $11 / 14 / 2000$ & Transparent & New & 718 & M & & & & \\
\hline Goodwin Island & $11 / 14 / 2000$ & Transparent & New & 704 & M & & & & \\
\hline Goodwin Island & $11 / 14 / 2000$ & Transparent & New & 704 & M & & & & \\
\hline Goodwin Island & $11 / 14 / 2000$ & Transparent & New & 722 & M & & & & \\
\hline Goodwin Island & $11 / 14 / 2000$ & Transparent & New & 716 & M & & & & \\
\hline Goodwin Island & $11 / 14 / 2000$ & Transparent & New & 704 & M & & & & \\
\hline Goodwin Island & $11 / 14 / 2000$ & Transparent & New & 704 & M & & & & \\
\hline Goodwin Island & $11 / 14 / 2000$ & Transparent & New & 702 & M & & & & \\
\hline Goodwin Island & $11 / 14 / 2000$ & Transparent & New & 684 & M & & & & \\
\hline Goodwin Island & $11 / 14 / 2000$ & Transparent & New & 720 & M & & & & \\
\hline Goodwin Island & $11 / 14 / 2000$ & Brown & Old & 702 & M & & & & \\
\hline Goodwin Island & $11 / 14 / 2000$ & Brown & Old & 700 & M & & & & \\
\hline Goodwin Island & $11 / 14 / 2000$ & Brown & Old & 700 & M & & & & \\
\hline Goodwin Island & $11 / 14 / 2000$ & Brown & Old & 720 & M & & & & \\
\hline Goodwin Island & $11 / 14 / 2000$ & Brown & Old & 722 & M & & & & \\
\hline Goodwin Island & $11 / 14 / 2000$ & Brown & Old & 700 & M & & & & \\
\hline Goodwin Island & $11 / 14 / 2000$ & Brown-white & Old & 706 & M & & & & \\
\hline Goodwin Island & $11 / 14 / 2000$ & Brown-white & Old & 696 & M & & & & \\
\hline Guinea Marsh & $11 / 14 / 2000$ & Trans-brown & Intermediate & 710 & M & 713 & & & \\
\hline Guinea Marsh & $11 / 14 / 2000$ & Trans-white & Intermediate/old & 700 & M & & & & \\
\hline Guinea Marsh & $11 / 14 / 2000$ & Trans-white & Intermediate/old & 724 & M & & & & \\
\hline Guinea Marsh & $11 / 14 / 2000$ & Trans-white & Intermediate/old & 702 & M & & & & \\
\hline Guinea Marsh & $11 / 14 / 2000$ & Transparent & New & 700 & M & & & & \\
\hline Guinea Marsh & $11 / 14 / 2000$ & Transparent & New & 724 & M & & & & \\
\hline
\end{tabular}


Appendix A (continued)

\begin{tabular}{|c|c|c|c|c|c|c|c|c|c|}
\hline Site & Date & Color & Age & $\begin{array}{l}\text { Size } \\
(\mu \mathrm{m})\end{array}$ & Sex & $\begin{array}{l}\text { Mean size } \\
\text { Guinea }(\mu \mathrm{m})\end{array}$ & $\begin{array}{l}\text { Mean size } \\
\text { Goodwin }(\mu \mathrm{m})\end{array}$ & $\begin{array}{l}\text { Mean size } \\
\text { Dameron }(\mu \mathrm{m})\end{array}$ & $\begin{array}{l}\text { Grand mean } \\
(\mu \mathrm{m})\end{array}$ \\
\hline Guinea Marsh & $11 / 14 / 2000$ & Transparent & New & 680 & M & & & & \\
\hline Guinea Marsh & $11 / 14 / 2000$ & Brown & Old & 718 & M & & & & \\
\hline Guinea Marsh & $11 / 14 / 2000$ & Brown & Old & 738 & M & & & & \\
\hline Guinea Marsh & $11 / 14 / 2000$ & Brown & Old & 720 & M & & & & \\
\hline Guinea Marsh & $11 / 14 / 2000$ & Brown & Old & 720 & M & & & & \\
\hline Guinea Marsh & $11 / 14 / 2000$ & Brown & Old & 720 & M & & & & \\
\hline Guinea Marsh & $11 / 14 / 2000$ & Brown & Old & 726 & M & & & & \\
\hline Guinea Marsh & $11 / 14 / 2000$ & Brown & Old & 700 & M & & & & \\
\hline Goodwin Island & $12 / 18 / 2000$ & Trans-brown & Intermediate & 704 & M & & 708.5217391 & & 708 \\
\hline Goodwin Island & $12 / 18 / 2000$ & Trans-brown & Intermediate & 720 & M & & & & \\
\hline Goodwin Island & $12 / 18 / 2000$ & Trans-brown & Intermediate & 680 & M & & & & \\
\hline Goodwin Island & $12 / 18 / 2000$ & Trans-brown & Intermediate & 710 & M & & & & \\
\hline Goodwin Island & $12 / 18 / 2000$ & Trans-brown & Intermediate & 720 & M & & & & \\
\hline Goodwin Island & $12 / 18 / 2000$ & Trans-brown & Intermediate & 700 & M & & & & \\
\hline Goodwin Island & $12 / 18 / 2000$ & Transparent & New & 700 & M & & & & \\
\hline Goodwin Island & $12 / 18 / 2000$ & Transparent & New & 700 & M & & & & \\
\hline Goodwin Island & $12 / 18 / 2000$ & Transparent & New & 704 & M & & & & \\
\hline Goodwin Island & $12 / 18 / 2000$ & Transparent & New & 722 & M & & & & \\
\hline Goodwin Island & $12 / 18 / 2000$ & Transparent & New & 712 & M & & & & \\
\hline Goodwin Island & $12 / 18 / 2000$ & Transparent & New & 702 & M & & & & \\
\hline Goodwin Island & $12 / 18 / 2000$ & Transparent & New & 690 & M & & & & \\
\hline Goodwin Island & $12 / 18 / 2000$ & Transparent & New & 720 & M & & & & \\
\hline Goodwin Island & $12 / 18 / 2000$ & Transparent & New & 740 & M & & & & \\
\hline Goodwin Island & $12 / 18 / 2000$ & Transparent & New & 700 & M & & & & \\
\hline Goodwin Island & $12 / 18 / 2000$ & Transparent & New & 700 & M & & & & \\
\hline Goodwin Island & $12 / 18 / 2000$ & Transparent & New & 726 & M & & & & \\
\hline Goodwin Island & $12 / 18 / 2000$ & Transparent & New & 706 & M & & & & \\
\hline Goodwin Island & $12 / 18 / 2000$ & Transparent & New & 698 & M & & & & \\
\hline Goodwin Island & $12 / 18 / 2000$ & Transparent & New & 720 & M & & & & \\
\hline Goodwin Island & $12 / 18 / 2000$ & Brown & Old & 702 & M & & & & \\
\hline Goodwin Island & $12 / 18 / 2000$ & Very brown & Very old & 720 & M & & & & \\
\hline Guinea Marsh & $12 / 18 / 2000$ & Trans-brown & Intermediate & 696 & M & 707.75 & & & \\
\hline Guinea Marsh & $12 / 18 / 2000$ & Trans-brown & Intermediate & 720 & M & & & & \\
\hline Guinea Marsh & $12 / 18 / 2000$ & Trans-brown & Intermediate & 720 & M & & & & \\
\hline Guinea Marsh & $12 / 18 / 2000$ & Trans-brown & Intermediate & 720 & M & & & & \\
\hline Guinea Marsh & $12 / 18 / 2000$ & Trans-brown & Intermediate & 724 & M & & & & \\
\hline Guinea Marsh & $12 / 18 / 2000$ & Trans-brown & Intermediate & 704 & M & & & & \\
\hline Guinea Marsh & $12 / 18 / 2000$ & Trans-white & Intermediate/old & 700 & M & & & & \\
\hline Guinea Marsh & $12 / 18 / 2000$ & Trans-white & Intermediate/old & 698 & M & & & & \\
\hline Guinea Marsh & $12 / 18 / 2000$ & Trans-white & Intermediate/old & 702 & M & & & & \\
\hline Guinea Marsh & $12 / 18 / 2000$ & Trans-white & Intermediate/old & 720 & M & & & & \\
\hline Guinea Marsh & $12 / 18 / 2000$ & Trans-white & Intermediate/old & 730 & M & & & & \\
\hline Guinea Marsh & $12 / 18 / 2000$ & Transparent & New & 708 & M & & & & \\
\hline Guinea Marsh & $12 / 18 / 2000$ & Transparent & New & 684 & M & & & & \\
\hline Guinea Marsh & $12 / 18 / 2000$ & Transparent & New & 702 & M & & & & \\
\hline Guinea Marsh & $12 / 18 / 2000$ & Transparent & New & 720 & M & & & & \\
\hline Guinea Marsh & $12 / 18 / 2000$ & Transparent & New & 700 & M & & & & \\
\hline Guinea Marsh & $12 / 18 / 2000$ & Transparent & New & 716 & M & & & & \\
\hline Guinea Marsh & $12 / 18 / 2000$ & Brown & Old & 700 & M & & & & \\
\hline Guinea Marsh & $12 / 18 / 2000$ & Brown & Old & 708 & M & & & & \\
\hline Guinea Marsh & $12 / 18 / 2000$ & Brown & Old & 710 & M & & & & \\
\hline Guinea Marsh & $12 / 18 / 2000$ & Brown & Old & 700 & M & & & & \\
\hline Guinea Marsh & $12 / 18 / 2000$ & Brown & Old & 720 & M & & & & \\
\hline
\end{tabular}


Appendix A (continued)

\begin{tabular}{|c|c|c|c|c|c|c|c|c|c|}
\hline Site & Date & Color & Age & $\begin{array}{l}\text { Size } \\
(\mu \mathrm{m})\end{array}$ & Sex & $\begin{array}{l}\text { Mean size } \\
\text { Guinea }(\mu \mathrm{m})\end{array}$ & $\begin{array}{l}\text { Mean size } \\
\text { Goodwin }(\mu \mathrm{m})\end{array}$ & $\begin{array}{l}\text { Mean size } \\
\text { Dameron }(\mu \mathrm{m})\end{array}$ & $\begin{array}{l}\text { Grand mean } \\
(\mu \mathrm{m})\end{array}$ \\
\hline Guinea Marsh & $12 / 18 / 2000$ & Brown & Old & 710 & M & & & & \\
\hline Guinea Marsh & $12 / 18 / 2000$ & Brown & Old & 680 & M & & & & \\
\hline Guinea Marsh & $12 / 18 / 2000$ & Brown & Old & 720 & M & & & & \\
\hline Guinea Marsh & $12 / 18 / 2000$ & Brown & Old & 720 & M & & & & \\
\hline Guinea Marsh & $12 / 18 / 2000$ & Brown & Old & 716 & M & & & & \\
\hline Guinea Marsh & $12 / 18 / 2000$ & Brown & Old & 720 & M & & & & \\
\hline Guinea Marsh & $12 / 18 / 2000$ & Brown & Old & 696 & M & & & & \\
\hline Guinea Marsh & $12 / 18 / 2000$ & Brown & Old & 720 & M & & & & \\
\hline Guinea Marsh & $12 / 18 / 2000$ & Brown & Old & 722 & M & & & & \\
\hline Guinea Marsh & $12 / 18 / 2000$ & Brown & Old & 700 & M & & & & \\
\hline Guinea Marsh & $12 / 18 / 2000$ & Brown-white & Old & 704 & M & & & & \\
\hline Guinea Marsh & $12 / 18 / 2000$ & Brown-white & Old & 700 & M & & & & \\
\hline Guinea Marsh & $12 / 18 / 2000$ & Brown-white & Old & 700 & M & & & & \\
\hline Guinea Marsh & $12 / 18 / 2000$ & Brown-white & Old & 700 & M & & & & \\
\hline Guinea Marsh & $12 / 18 / 2000$ & Very brown & Very old & 700 & M & & & & \\
\hline Guinea Marsh & $12 / 18 / 2000$ & White-opaque & Very old & 700 & M & & & & \\
\hline Guinea Marsh & $12 / 18 / 2000$ & White-opaque & Very old & 720 & M & & & & \\
\hline Guinea Marsh & $12 / 18 / 2000$ & White-opaque & Very old & 730 & M & & & & \\
\hline Guinea Marsh & $12 / 18 / 2000$ & White-opaque & Very old & 684 & M & & & & \\
\hline Guinea Marsh & $12 / 18 / 2000$ & White-opaque & Very old & 720 & M & & & & \\
\hline Guinea Marsh & $12 / 18 / 2000$ & White-opaque & Very old & 680 & M & & & & \\
\hline Guinea Marsh & $12 / 18 / 2000$ & White-opaque & Very old & 696 & M & & & & \\
\hline Guinea Marsh & $12 / 18 / 2000$ & White-opaque & Very old & 686 & M & & & & \\
\hline Guinea Marsh & $12 / 18 / 2000$ & White-opaque & Very old & 700 & M & & & & \\
\hline Guinea Marsh & $12 / 18 / 2000$ & White-opaque & Very old & 722 & M & & & & \\
\hline Guinea Marsh & $12 / 18 / 2000$ & White-opaque & Very old & 724 & M & & & & \\
\hline Goodwin Island & 2/9/1999 & Brown & Old & 610 & F & & 602 & & 605.6 \\
\hline Goodwin Island & 2/9/1999 & Brown & Old & 614 & F & & & & \\
\hline Goodwin Island & 2/9/1999 & Brown & Old & 598 & $\mathrm{~F}$ & & & & \\
\hline Goodwin Island & 2/9/1999 & Brown & Old & 588 & $\mathrm{~F}$ & & & & \\
\hline Goodwin Island & 2/9/1999 & Brown-opaque & Very old & 600 & F & & & & \\
\hline Guinea Marsh & 2/9/1999 & Brown-white & Old & 628 & $\mathrm{~F}$ & 609.2 & & & \\
\hline Guinea Marsh & 2/9/1999 & Trans-brown & Intermediate & 612 & $\mathrm{~F}$ & & & & \\
\hline Guinea Marsh & 2/9/1999 & Trans-brown & Intermediate & 624 & $\mathrm{~F}$ & & & & \\
\hline Guinea Marsh & 2/9/1999 & Very brown & Very old & 582 & $\mathrm{~F}$ & & & & \\
\hline Guinea Marsh & 2/9/1999 & Very brown & Very old & 600 & $\mathrm{~F}$ & & & & \\
\hline Guinea Marsh & 3/30/1999 & Trans-brown & Intermediate & 624 & $\mathrm{~F}$ & 624 & & & 624 \\
\hline Goodwin Island & 4/27/1999 & Brown-white & Old & 628 & $\mathrm{~F}$ & & 628 & & 617.9090909 \\
\hline Guinea Marsh & 4/27/1999 & Brown & Old & 624 & F & 617.4285714 & & & \\
\hline Guinea Marsh & 4/27/1999 & Brown & Old & 640 & $\mathrm{~F}$ & & & & \\
\hline Guinea Marsh & 4/27/1999 & Brown & Old & 604 & $\mathrm{~F}$ & & & & \\
\hline Guinea Marsh & 4/27/1999 & Brown & Old & 628 & F & & & & \\
\hline Guinea Marsh & 4/27/1999 & Brown & Old & 606 & $\mathrm{~F}$ & & & & \\
\hline Guinea Marsh & 4/27/1999 & Brown & Old & 606 & $\mathrm{~F}$ & & & & \\
\hline Guinea Marsh & 4/27/1999 & Brown & Old & 608 & F & & & & \\
\hline Guinea Marsh & 4/27/1999 & Brown & Old & 604 & F & & & & \\
\hline Guinea Marsh & 4/27/1999 & Brown & Old & 596 & $\mathrm{~F}$ & & & & \\
\hline Guinea Marsh & 4/27/1999 & Brown-opaque & Very old & 632 & $\mathrm{~F}$ & & & & \\
\hline Guinea Marsh & 4/27/1999 & Brown-white & Old & 626 & F & & & & \\
\hline Guinea Marsh & 4/27/1999 & Brown-white & Old & 628 & $\mathrm{~F}$ & & & & \\
\hline Guinea Marsh & 4/27/1999 & Trans-brown & Intermediate & 624 & $\mathrm{~F}$ & & & & \\
\hline Guinea Marsh & 4/27/1999 & Trans-brown & Intermediate & 620 & F & & & & \\
\hline Guinea Marsh & 4/27/1999 & Trans-brown & Intermediate & 602 & $\mathrm{~F}$ & & & & \\
\hline
\end{tabular}




\begin{tabular}{|c|c|c|c|c|c|c|c|c|c|}
\hline Site & Date & Color & Age & $\begin{array}{l}\text { Size } \\
(\mu \mathrm{m})\end{array}$ & Sex & $\begin{array}{l}\text { Mean size } \\
\text { Guinea }(\mu \mathrm{m})\end{array}$ & $\begin{array}{l}\text { Mean size } \\
\text { Goodwin }(\mu \mathrm{m})\end{array}$ & $\begin{array}{l}\text { Mean size } \\
\text { Dameron }(\mu \mathrm{m})\end{array}$ & $\begin{array}{l}\text { Grand mean } \\
\text { size }(\mu \mathrm{m})\end{array}$ \\
\hline Guinea Marsh & 4/27/1999 & Trans-brown & Intermediate & 620 & $\mathrm{~F}$ & & & & \\
\hline Guinea Marsh & $4 / 27 / 1999$ & Trans-brown & Intermediate & 626 & $\mathrm{~F}$ & & & & \\
\hline Guinea Marsh & $4 / 27 / 1999$ & Trans-brown & Intermediate & 620 & $\mathrm{~F}$ & & & & \\
\hline Guinea Marsh & $4 / 27 / 1999$ & Trans-brown & Intermediate & 630 & $\mathrm{~F}$ & & & & \\
\hline Guinea Marsh & $4 / 27 / 1999$ & Transparent & New & 606 & $\mathrm{~F}$ & & & & \\
\hline Guinea Marsh & $4 / 27 / 1999$ & Very brown/opaque & Very old & 616 & $\mathrm{~F}$ & & & & \\
\hline Goodwin Island & $5 / 12 / 1999$ & Brown & Old & 616 & $\mathrm{~F}$ & & 607.3333333 & & 630.0869565 \\
\hline Goodwin Island & $5 / 12 / 1999$ & Brown-opaque & Very old & 600 & $\mathrm{~F}$ & & & & \\
\hline Goodwin Island & $5 / 12 / 1999$ & Very brown & Very old & 606 & $\mathrm{~F}$ & & & & \\
\hline Guinea Marsh & $5 / 12 / 1999$ & Brown & Old & 614 & $\mathrm{~F}$ & 633.5 & & & \\
\hline Guinea Marsh & $5 / 12 / 1999$ & Brown & Old & 628 & $\mathrm{~F}$ & & & & \\
\hline Guinea Marsh & $5 / 12 / 1999$ & Brown-opaque & Very old & 620 & $\mathrm{~F}$ & & & & \\
\hline Guinea Marsh & $5 / 12 / 1999$ & Brown-opaque & Very old & 610 & $\mathrm{~F}$ & & & & \\
\hline Guinea Marsh & $5 / 12 / 1999$ & Brown-red & Very old & 608 & $\mathrm{~F}$ & & & & \\
\hline Guinea Marsh & $5 / 12 / 1999$ & Transparent & New & 630 & $\mathrm{~F}$ & & & & \\
\hline Guinea Marsh & $5 / 12 / 1999$ & Transparent & New & 626 & $\mathrm{~F}$ & & & & \\
\hline Guinea Marsh & $5 / 12 / 1999$ & Transparent & New & 632 & $\mathrm{~F}$ & & & & \\
\hline Guinea Marsh & $5 / 12 / 1999$ & Transparent & New & 638 & $\mathrm{~F}$ & & & & \\
\hline Guinea Marsh & $5 / 12 / 1999$ & Transparent & New & 640 & $\mathrm{~F}$ & & & & \\
\hline Guinea Marsh & $5 / 12 / 1999$ & Transparent & New & 646 & $\mathrm{~F}$ & & & & \\
\hline Guinea Marsh & $5 / 12 / 1999$ & Transparent & New & 648 & $\mathrm{~F}$ & & & & \\
\hline Guinea Marsh & $5 / 12 / 1999$ & Transparent & New & 644 & $\mathrm{~F}$ & & & & \\
\hline Guinea Marsh & $5 / 12 / 1999$ & Transparent & New & 640 & $\mathrm{~F}$ & & & & \\
\hline Guinea Marsh & $5 / 12 / 1999$ & Transparent & New & 644 & $\mathrm{~F}$ & & & & \\
\hline Guinea Marsh & $5 / 12 / 1999$ & Trans-red & New & 636 & $\mathrm{~F}$ & & & & \\
\hline Guinea Marsh & $5 / 12 / 1999$ & Trans-red & New & 656 & $\mathrm{~F}$ & & & & \\
\hline Guinea Marsh & $5 / 12 / 1999$ & Trans-red & New & 630 & $\mathrm{~F}$ & & & & \\
\hline Guinea Marsh & $5 / 12 / 1999$ & Trans-red & New & 644 & $\mathrm{~F}$ & & & & \\
\hline Guinea Marsh & $5 / 12 / 1999$ & White-opaque & Very old & 636 & $\mathrm{~F}$ & & & & \\
\hline Goodwin Island & 6/3/1999 & Trans-brown & Intermediate & 640 & $\mathrm{~F}$ & & 640 & & 628.6666667 \\
\hline Guinea Marsh & 6/3/1999 & Trans-white & Intermediate/old & 624 & $\mathrm{~F}$ & 623 & & & \\
\hline Guinea Marsh & 6/3/1999 & Trans-white & Intermediate/old & 622 & $\mathrm{~F}$ & & & & \\
\hline Goodwin Island & $6 / 22 / 1999$ & Brown & Old & 604 & $\mathrm{~F}$ & & 621 & & 625.3846154 \\
\hline Goodwin Island & $6 / 22 / 1999$ & Trans-brown & Intermediate & 640 & $\mathrm{~F}$ & & & & \\
\hline Goodwin Island & $6 / 22 / 1999$ & Transparent & New & 624 & $\mathrm{~F}$ & & & & \\
\hline Goodwin Island & $6 / 22 / 1999$ & Transparent & New & 616 & $\mathrm{~F}$ & & & & \\
\hline Guinea Marsh & $6 / 22 / 1999$ & Brown & Old & 626 & $\mathrm{~F}$ & 625.8857143 & & & \\
\hline Guinea Marsh & $6 / 22 / 1999$ & Brown & Old & 636 & $\mathrm{~F}$ & & & & \\
\hline Guinea Marsh & $6 / 22 / 1999$ & Brown & Old & 634 & $\mathrm{~F}$ & & & & \\
\hline Guinea Marsh & $6 / 22 / 1999$ & Brown & Old & 600 & $\mathrm{~F}$ & & & & \\
\hline Guinea Marsh & $6 / 22 / 1999$ & Brown & Old & 614 & $\mathrm{~F}$ & & & & \\
\hline Guinea Marsh & $6 / 22 / 1999$ & Brown & Old & 620 & $\mathrm{~F}$ & & & & \\
\hline Guinea Marsh & $6 / 22 / 1999$ & Brown & Old & 624 & $\mathrm{~F}$ & & & & \\
\hline Guinea Marsh & $6 / 22 / 1999$ & Brown & Old & 632 & $\mathrm{~F}$ & & & & \\
\hline Guinea Marsh & $6 / 22 / 1999$ & Brown & Old & 620 & $\mathrm{~F}$ & & & & \\
\hline Guinea Marsh & $6 / 22 / 1999$ & Brown & Old & 620 & $\mathrm{~F}$ & & & & \\
\hline Guinea Marsh & $6 / 22 / 1999$ & Brown & Old & 622 & $\mathrm{~F}$ & & & & \\
\hline Guinea Marsh & $6 / 22 / 1999$ & Brown & Old & 628 & $\mathrm{~F}$ & & & & \\
\hline Guinea Marsh & $6 / 22 / 1999$ & Brown & Old & 624 & $\mathrm{~F}$ & & & & \\
\hline Guinea Marsh & $6 / 22 / 1999$ & Brown-opaque & Very old & 646 & $\mathrm{~F}$ & & & & \\
\hline Guinea Marsh & $6 / 22 / 1999$ & Trans-brown & Intermediate & 626 & $\mathrm{~F}$ & & & & \\
\hline Guinea Marsh & $6 / 22 / 1999$ & Trans-brown & Intermediate & 620 & $\mathrm{~F}$ & & & & \\
\hline Guinea Marsh & $6 / 22 / 1999$ & Trans-brown & Intermediate & 646 & $\mathrm{~F}$ & & & & \\
\hline
\end{tabular}


Appendix A (continued)

\begin{tabular}{|c|c|c|c|c|c|c|c|c|c|}
\hline Site & Date & Color & Age & $\begin{array}{l}\text { Size } \\
(\mu \mathrm{m})\end{array}$ & Sex & $\begin{array}{l}\text { Mean size } \\
\text { Guinea }(\mu \mathrm{m})\end{array}$ & $\begin{array}{l}\text { Mean size } \\
\text { Goodwin }(\mu \mathrm{m})\end{array}$ & $\begin{array}{l}\text { Mean size } \\
\text { Dameron }(\mu \mathrm{m})\end{array}$ & $\begin{array}{l}\text { Grand mean } \\
\text { size }(\mu \mathrm{m})\end{array}$ \\
\hline Guinea Marsh & $6 / 22 / 1999$ & Trans-brown & Intermediate & 620 & $\mathrm{~F}$ & & & & \\
\hline Guinea Marsh & $6 / 22 / 1999$ & Trans-brown & Intermediate & 640 & $\mathrm{~F}$ & & & & \\
\hline Guinea Marsh & $6 / 22 / 1999$ & Transparent & New & 608 & F & & & & \\
\hline Guinea Marsh & $6 / 22 / 1999$ & Transparent & New & 618 & $\mathrm{~F}$ & & & & \\
\hline Guinea Marsh & $6 / 22 / 1999$ & Transparent & New & 616 & $\mathrm{~F}$ & & & & \\
\hline Guinea Marsh & $6 / 22 / 1999$ & Transparent & New & 622 & F & & & & \\
\hline Guinea Marsh & $6 / 22 / 1999$ & Transparent & New & 624 & $\mathrm{~F}$ & & & & \\
\hline Guinea Marsh & $6 / 22 / 1999$ & Transparent & New & 616 & F & & & & \\
\hline Guinea Marsh & $6 / 22 / 1999$ & Transparent & New & 614 & $\mathrm{~F}$ & & & & \\
\hline Guinea Marsh & $6 / 22 / 1999$ & Transparent & New & 610 & $\mathrm{~F}$ & & & & \\
\hline Guinea Marsh & $6 / 22 / 1999$ & Trans-yellow-brown & Old & 632 & $\mathrm{~F}$ & & & & \\
\hline Guinea Marsh & $6 / 22 / 1999$ & Very brown & Very old & 620 & $\mathrm{~F}$ & & & & \\
\hline Guinea Marsh & $6 / 22 / 1999$ & Very brown & Very old & 652 & F & & & & \\
\hline Guinea Marsh & $6 / 22 / 1999$ & Very brown & Very old & 636 & $\mathrm{~F}$ & & & & \\
\hline Guinea Marsh & $6 / 22 / 1999$ & Very brown & Very old & 638 & $\mathrm{~F}$ & & & & \\
\hline Guinea Marsh & $6 / 22 / 1999$ & Very brown & Very old & 640 & F & & & & \\
\hline Guinea Marsh & $6 / 22 / 1999$ & Very brown & Very old & 634 & F & & & & \\
\hline Guinea Marsh & $6 / 22 / 1999$ & Very brown & Very old & 628 & $\mathrm{~F}$ & & & & \\
\hline Goodwin Island & 7/6/1999 & Brown & Old & 620 & F & & 612.4 & & 616.6086957 \\
\hline Goodwin Island & 7/6/1999 & Brown & Old & 624 & $\mathrm{~F}$ & & & & \\
\hline Goodwin Island & $7 / 6 / 1999$ & Brown & Old & 608 & F & & & & \\
\hline Goodwin Island & 7/6/1999 & Brown & Old & 618 & $\mathrm{~F}$ & & & & \\
\hline Goodwin Island & $7 / 6 / 1999$ & Brown & Old & 620 & $\mathrm{~F}$ & & & & \\
\hline Goodwin Island & 7/6/1999 & Brown-opaque & Very old & 604 & F & & & & \\
\hline Goodwin Island & $7 / 6 / 1999$ & Brown-opaque & Very old & 618 & $\mathrm{~F}$ & & & & \\
\hline Goodwin Island & $7 / 6 / 1999$ & Brown-opaque & Very old & 600 & $\mathrm{~F}$ & & & & \\
\hline Goodwin Island & 7/6/1999 & Trans-brown & Intermediate & 602 & F & & & & \\
\hline Goodwin Island & $7 / 6 / 1999$ & Transparent & New & 610 & $\mathrm{~F}$ & & & & \\
\hline Guinea Marsh & $7 / 6 / 1999$ & Brown & Old & 642 & F & 619.8461538 & & & \\
\hline Guinea Marsh & 7/6/1999 & Brown & Old & 624 & F & & & & \\
\hline Guinea Marsh & 7/6/1999 & Brown & Old & 626 & F & & & & \\
\hline Guinea Marsh & 7/6/1999 & Trans-brown & Intermediate & 640 & F & & & & \\
\hline Guinea Marsh & 7/6/1999 & Trans-brown & Intermediate & 622 & F & & & & \\
\hline Guinea Marsh & $7 / 6 / 1999$ & Trans-brown & Intermediate & 616 & $\mathrm{~F}$ & & & & \\
\hline Guinea Marsh & 7/6/1999 & Trans-brown & Intermediate & 614 & F & & & & \\
\hline Guinea Marsh & 7/6/1999 & Trans-brown & Intermediate & 606 & F & & & & \\
\hline Guinea Marsh & $7 / 6 / 1999$ & Transparent & New & 614 & $\mathrm{~F}$ & & & & \\
\hline Guinea Marsh & 7/6/1999 & Transparent & New & 604 & F & & & & \\
\hline Guinea Marsh & 7/6/1999 & Transparent & New & 626 & $\mathrm{~F}$ & & & & \\
\hline Guinea Marsh & $7 / 6 / 1999$ & Transparent & New & 606 & $\mathrm{~F}$ & & & & \\
\hline Guinea Marsh & 7/6/1999 & Transparent & New & 618 & F & & & & \\
\hline Goodwin Island & $7 / 20 / 1999$ & Brown & Old & 596 & F & & 605.7241379 & & 608.9090909 \\
\hline Goodwin Island & $7 / 20 / 1999$ & Brown & Old & 602 & F & & & & \\
\hline Goodwin Island & $7 / 20 / 1999$ & Brown & Old & 622 & F & & & & \\
\hline Goodwin Island & $7 / 20 / 1999$ & Brown & Old & 620 & $\mathrm{~F}$ & & & & \\
\hline Goodwin Island & $7 / 20 / 1999$ & Brown & Old & 624 & F & & & & \\
\hline Goodwin Island & $7 / 20 / 1999$ & Brown & Old & 620 & F & & & & \\
\hline Goodwin Island & $7 / 20 / 1999$ & Brown & Old & 624 & $\mathrm{~F}$ & & & & \\
\hline Goodwin Island & $7 / 20 / 1999$ & Brown & Old & 638 & F & & & & \\
\hline Goodwin Island & $7 / 20 / 1999$ & Brown & Old & 600 & $\mathrm{~F}$ & & & & \\
\hline Goodwin Island & $7 / 20 / 1999$ & Brown & Old & 608 & $\mathrm{~F}$ & & & & \\
\hline Goodwin Island & $7 / 20 / 1999$ & Brown-opaque & Very old & 612 & F & & & & \\
\hline Goodwin Island & $7 / 20 / 1999$ & Brown-opaque & Very old & 622 & $\mathrm{~F}$ & & & & \\
\hline Goodwin Island & $7 / 20 / 1999$ & Brown-opaque & Very old & 616 & $\mathrm{~F}$ & & & & \\
\hline
\end{tabular}


Appendix A (continued)

\begin{tabular}{|c|c|c|c|c|c|c|c|c|c|}
\hline Site & Date & Color & Age & $\begin{array}{l}\text { Size } \\
(\mu \mathrm{m})\end{array}$ & Sex & $\begin{array}{l}\text { Mean size } \\
\text { Guinea }(\mu \mathrm{m})\end{array}$ & $\begin{array}{l}\text { Mean size } \\
\text { Goodwin }(\mu \mathrm{m})\end{array}$ & $\begin{array}{l}\text { Mean size } \\
\text { Dameron }(\mu \mathrm{m})\end{array}$ & $\begin{array}{l}\text { Grand mean } \\
\text { size }(\mu \mathrm{m})\end{array}$ \\
\hline Goodwin Island & 7/20/1999 & Opaque & Very old & 622 & $\mathrm{~F}$ & & & & \\
\hline Goodwin Island & $7 / 20 / 1999$ & Trans-brown & Intermediate & 604 & $\mathrm{~F}$ & & & & \\
\hline Goodwin Island & 7/20/1999 & Trans-brown & Intermediate & 590 & $\mathrm{~F}$ & & & & \\
\hline Goodwin Island & 7/20/1999 & Trans-brown & Intermediate & 538 & $\mathrm{~F}$ & & & & \\
\hline Goodwin Island & 7/20/1999 & Trans-brown & Intermediate & 580 & $\mathrm{~F}$ & & & & \\
\hline Goodwin Island & 7/20/1999 & Trans-brown & Intermediate & 582 & $\mathrm{~F}$ & & & & \\
\hline Goodwin Island & 7/20/1999 & Trans-brown & Intermediate & 602 & $\mathrm{~F}$ & & & & \\
\hline Goodwin Island & 7/20/1999 & Trans-brown & Intermediate & 620 & $\mathrm{~F}$ & & & & \\
\hline Goodwin Island & 7/20/1999 & Trans-brown & Intermediate & 596 & $\mathrm{~F}$ & & & & \\
\hline Goodwin Island & 7/20/1999 & Trans-brown & Intermediate & 606 & $\mathrm{~F}$ & & & & \\
\hline Goodwin Island & 7/20/1999 & Trans-brown & Intermediate & 602 & $\mathrm{~F}$ & & & & \\
\hline Goodwin Island & 7/20/1999 & Trans-brown & Intermediate & 602 & $\mathrm{~F}$ & & & & \\
\hline Goodwin Island & 7/20/1999 & Trans-opaque & Intermediate & 604 & $\mathrm{~F}$ & & & & \\
\hline Goodwin Island & 7/20/1999 & Transparent & New & 606 & $\mathrm{~F}$ & & & & \\
\hline Goodwin Island & 7/20/1999 & Transparent & New & 606 & $\mathrm{~F}$ & & & & \\
\hline Goodwin Island & 7/20/1999 & Transparent & New & 602 & $\mathrm{~F}$ & & & & \\
\hline Guinea Marsh & 7/20/1999 & Brown & Old & 624 & $\mathrm{~F}$ & 610.4745763 & & & \\
\hline Guinea Marsh & 7/20/1999 & Brown & Old & 618 & $\mathrm{~F}$ & & & & \\
\hline Guinea Marsh & 7/20/1999 & Brown & Old & 590 & $\mathrm{~F}$ & & & & \\
\hline Guinea Marsh & 7/20/1999 & Brown & Old & 598 & $\mathrm{~F}$ & & & & \\
\hline Guinea Marsh & 7/20/1999 & Brown & Old & 620 & $\mathrm{~F}$ & & & & \\
\hline Guinea Marsh & 7/20/1999 & Brown & Old & 614 & $\mathrm{~F}$ & & & & \\
\hline Guinea Marsh & 7/20/1999 & Brown & Old & 626 & $\mathrm{~F}$ & & & & \\
\hline Guinea Marsh & 7/20/1999 & Brown & Old & 622 & $\mathrm{~F}$ & & & & \\
\hline Guinea Marsh & 7/20/1999 & Brown & Old & 632 & $\mathrm{~F}$ & & & & \\
\hline Guinea Marsh & 7/20/1999 & Brown & Old & 642 & $\mathrm{~F}$ & & & & \\
\hline Guinea Marsh & 7/20/1999 & Brown & Old & 620 & $\mathrm{~F}$ & & & & \\
\hline Guinea Marsh & 7/20/1999 & Brown & Old & 622 & $\mathrm{~F}$ & & & & \\
\hline Guinea Marsh & 7/20/1999 & Brown & Old & 620 & $\mathrm{~F}$ & & & & \\
\hline Guinea Marsh & 7/20/1999 & Brown & Old & 610 & $\mathrm{~F}$ & & & & \\
\hline Guinea Marsh & 7/20/1999 & Brown & Old & 628 & $\mathrm{~F}$ & & & & \\
\hline Guinea Marsh & 7/20/1999 & Brown-opaque & Very old & 636 & $\mathrm{~F}$ & & & & \\
\hline Guinea Marsh & 7/20/1999 & Trans-brown & Intermediate & 580 & $\mathrm{~F}$ & & & & \\
\hline Guinea Marsh & 7/20/1999 & Trans-brown & Intermediate & 622 & $\mathrm{~F}$ & & & & \\
\hline Guinea Marsh & 7/20/1999 & Trans-brown & Intermediate & 620 & $\mathrm{~F}$ & & & & \\
\hline Guinea Marsh & 7/20/1999 & Trans-brown & Intermediate & 604 & $\mathrm{~F}$ & & & & \\
\hline Guinea Marsh & 7/20/1999 & Trans-brown & Intermediate & 610 & $\mathrm{~F}$ & & & & \\
\hline Guinea Marsh & 7/20/1999 & Trans-brown & Intermediate & 596 & $\mathrm{~F}$ & & & & \\
\hline Guinea Marsh & 7/20/1999 & Trans-brown & Intermediate & 600 & $\mathrm{~F}$ & & & & \\
\hline Guinea Marsh & 7/20/1999 & Trans-brown & Intermediate & 606 & $\mathrm{~F}$ & & & & \\
\hline Guinea Marsh & 7/20/1999 & Trans-brown & Intermediate & 608 & $\mathrm{~F}$ & & & & \\
\hline Guinea Marsh & 7/20/1999 & Trans-brown & Intermediate & 608 & $\mathrm{~F}$ & & & & \\
\hline Guinea Marsh & 7/20/1999 & Trans-brown & Intermediate & 616 & $\mathrm{~F}$ & & & & \\
\hline Guinea Marsh & 7/20/1999 & Trans-brown & Intermediate & 620 & $\mathrm{~F}$ & & & & \\
\hline Guinea Marsh & 7/20/1999 & Trans-brown & Intermediate & 596 & $\mathrm{~F}$ & & & & \\
\hline Guinea Marsh & 7/20/1999 & Trans-brown & Intermediate & 614 & $\mathrm{~F}$ & & & & \\
\hline Guinea Marsh & 7/20/1999 & Trans-brown & Intermediate & 616 & $\mathrm{~F}$ & & & & \\
\hline Guinea Marsh & 7/20/1999 & Trans-brown & Intermediate & 602 & $\mathrm{~F}$ & & & & \\
\hline Guinea Marsh & 7/20/1999 & Trans-brown & Intermediate & 606 & $\mathrm{~F}$ & & & & \\
\hline Guinea Marsh & 7/20/1999 & Trans-brown & Intermediate & 612 & $\mathrm{~F}$ & & & & \\
\hline Guinea Marsh & 7/20/1999 & Trans-brown & Intermediate & 604 & $\mathrm{~F}$ & & & & \\
\hline Guinea Marsh & 7/20/1999 & Transparent & New & 620 & $\mathrm{~F}$ & & & & \\
\hline Guinea Marsh & 7/20/1999 & Transparent & New & 604 & $\mathrm{~F}$ & & & & \\
\hline
\end{tabular}


Appendix A (continued)

\begin{tabular}{|c|c|c|c|c|c|c|c|c|c|}
\hline Site & Date & Color & Age & $\begin{array}{l}\text { Size } \\
(\mu \mathrm{m})\end{array}$ & Sex & $\begin{array}{l}\text { Mean size } \\
\text { Guinea }(\mu \mathrm{m})\end{array}$ & $\begin{array}{l}\text { Mean size } \\
\text { Goodwin }(\mu \mathrm{m})\end{array}$ & $\begin{array}{l}\text { Mean size } \\
\text { Dameron }(\mu \mathrm{m})\end{array}$ & $\begin{array}{l}\text { Grand mean } \\
\text { size }(\mu \mathrm{m})\end{array}$ \\
\hline Guinea Marsh & $7 / 20 / 1999$ & Transparent & New & 602 & $\mathrm{~F}$ & & & & \\
\hline Guinea Marsh & $7 / 20 / 1999$ & Transparent & New & 600 & F & & & & \\
\hline Guinea Marsh & $7 / 20 / 1999$ & Transparent & New & 618 & F & & & & \\
\hline Guinea Marsh & $7 / 20 / 1999$ & Transparent & New & 600 & $\mathrm{~F}$ & & & & \\
\hline Guinea Marsh & $7 / 20 / 1999$ & Transparent & New & 612 & F & & & & \\
\hline Guinea Marsh & 7/20/1999 & Transparent & New & 608 & F & & & & \\
\hline Guinea Marsh & $7 / 20 / 1999$ & Transparent & New & 604 & F & & & & \\
\hline Guinea Marsh & $7 / 20 / 1999$ & Transparent & New & 604 & F & & & & \\
\hline Guinea Marsh & $7 / 20 / 1999$ & Transparent & New & 622 & $\mathrm{~F}$ & & & & \\
\hline Guinea Marsh & $7 / 20 / 1999$ & Transparent & New & 608 & F & & & & \\
\hline Guinea Marsh & $7 / 20 / 1999$ & Transparent & New & 612 & F & & & & \\
\hline Guinea Marsh & $7 / 20 / 1999$ & Transparent & New & 600 & $\mathrm{~F}$ & & & & \\
\hline Guinea Marsh & $7 / 20 / 1999$ & Transparent & New & 598 & F & & & & \\
\hline Guinea Marsh & $7 / 20 / 1999$ & Transparent & New & 596 & F & & & & \\
\hline Guinea Marsh & $7 / 20 / 1999$ & Transparent & New & 590 & $\mathrm{~F}$ & & & & \\
\hline Guinea Marsh & $7 / 20 / 1999$ & Transparent & New & 610 & F & & & & \\
\hline Guinea Marsh & $7 / 20 / 1999$ & Transparent & New & 614 & $\mathrm{~F}$ & & & & \\
\hline Guinea Marsh & $7 / 20 / 1999$ & Transparent & New & 622 & $\mathrm{~F}$ & & & & \\
\hline Guinea Marsh & $7 / 20 / 1999$ & Transparent & New & 604 & F & & & & \\
\hline Guinea Marsh & $7 / 20 / 1999$ & Transparent & New & 602 & $\mathrm{~F}$ & & & & \\
\hline Guinea Marsh & $7 / 20 / 1999$ & Transparent & New & 606 & F & & & & \\
\hline Guinea Marsh & $7 / 20 / 1999$ & Trans-yellow & Old & 600 & F & & & & \\
\hline Goodwin Island & $8 / 3 / 1999$ & Brown & Old & 622 & $\mathrm{~F}$ & & 622 & & 616.7407407 \\
\hline Goodwin Island & $8 / 3 / 1999$ & Brown-white & Old & 622 & F & & & & \\
\hline Goodwin Island & $8 / 3 / 1999$ & Brown-white & Old & 622 & $\mathrm{~F}$ & & & & \\
\hline Goodwin Island & $8 / 3 / 1999$ & Trans-brown & Intermediate & 622 & $\mathrm{~F}$ & & & & \\
\hline Goodwin Island & $8 / 3 / 1999$ & Trans-brown & Intermediate & 622 & F & & & & \\
\hline Goodwin Island & $8 / 3 / 1999$ & Trans-brown & Intermediate & 622 & $\mathrm{~F}$ & & & & \\
\hline Goodwin Island & $8 / 3 / 1999$ & Trans-brown & Intermediate & 622 & F & & & & \\
\hline Goodwin Island & $8 / 3 / 1999$ & Trans-brown & Intermediate & 622 & F & & & & \\
\hline Goodwin Island & $8 / 3 / 1999$ & Transparent & New & 622 & $\mathrm{~F}$ & & & & \\
\hline Goodwin Island & $8 / 3 / 1999$ & Transparent & New & 622 & F & & & & \\
\hline Guinea Marsh & 8/3/1999 & Brown & Old & 626 & F & 613.6470588 & & & \\
\hline Guinea Marsh & $8 / 3 / 1999$ & Brown & Old & 616 & $\mathrm{~F}$ & & & & \\
\hline Guinea Marsh & 8/3/1999 & Brown-opaque & Very old & 620 & F & & & & \\
\hline Guinea Marsh & 8/3/1999 & Brown-opaque & Very old & 602 & F & & & & \\
\hline Guinea Marsh & $8 / 3 / 1999$ & Trans-brown & Intermediate & 620 & $\mathrm{~F}$ & & & & \\
\hline Guinea Marsh & 8/3/1999 & Trans-brown & Intermediate & 624 & F & & & & \\
\hline Guinea Marsh & 8/3/1999 & Trans-brown & Intermediate & 606 & F & & & & \\
\hline Guinea Marsh & 8/3/1999 & Trans-brown & Intermediate & 606 & $\mathrm{~F}$ & & & & \\
\hline Guinea Marsh & 8/3/1999 & Trans-brown & Intermediate & 618 & F & & & & \\
\hline Guinea Marsh & 8/3/1999 & Trans-brown & Intermediate & 614 & F & & & & \\
\hline Guinea Marsh & 8/3/1999 & Trans-brown & Intermediate & 614 & F & & & & \\
\hline Guinea Marsh & 8/3/1999 & Trans-brown & Intermediate & 608 & F & & & & \\
\hline Guinea Marsh & $8 / 3 / 1999$ & Trans-brown & Intermediate & 614 & $\mathrm{~F}$ & & & & \\
\hline Guinea Marsh & 8/3/1999 & Trans-brown & Intermediate & 622 & F & & & & \\
\hline Guinea Marsh & 8/3/1999 & Transparent & New & 612 & F & & & & \\
\hline Guinea Marsh & $8 / 3 / 1999$ & Transparent & New & 604 & $\mathrm{~F}$ & & & & \\
\hline Guinea Marsh & 8/3/1999 & Transparent & New & 606 & F & & & & \\
\hline Goodwin Island & $8 / 17 / 1999$ & Brown & Old & 624 & F & & 603.4 & & 605.7777778 \\
\hline Goodwin Island & $8 / 17 / 1999$ & Brown & Old & 604 & $\mathrm{~F}$ & & & & \\
\hline Goodwin Island & $8 / 17 / 1999$ & Brown & Old & 620 & F & & & & \\
\hline Goodwin Island & $8 / 17 / 1999$ & Brown-opaque & Very old & 612 & $\mathrm{~F}$ & & & & \\
\hline Goodwin Island & $8 / 17 / 1999$ & Brown-opaque & Very old & 596 & $\mathrm{~F}$ & & & & \\
\hline
\end{tabular}




\begin{tabular}{|c|c|c|c|c|c|c|c|c|c|}
\hline Site & Date & Color & Age & $\begin{array}{l}\text { Size } \\
(\mu \mathrm{m})\end{array}$ & Sex & $\begin{array}{l}\text { Mean size } \\
\text { Guinea }(\mu \mathrm{m})\end{array}$ & $\begin{array}{l}\text { Mean size } \\
\text { Goodwin }(\mu \mathrm{m})\end{array}$ & $\begin{array}{l}\text { Mean size } \\
\text { Dameron }(\mu \mathrm{m})\end{array}$ & $\begin{array}{l}\text { Grand mean } \\
\text { size }(\mu \mathrm{m})\end{array}$ \\
\hline Goodwin Island & $8 / 17 / 1999$ & Transparent & New & 592 & $\mathrm{~F}$ & & & & \\
\hline Goodwin Island & 8/17/1999 & Transparent & New & 590 & $\mathrm{~F}$ & & & & \\
\hline Goodwin Island & 8/17/1999 & Transparent & New & 602 & $\mathrm{~F}$ & & & & \\
\hline Goodwin Island & $8 / 17 / 1999$ & Transparent & New & 604 & $\mathrm{~F}$ & & & & \\
\hline Goodwin Island & 8/17/1999 & Transparent & New & 590 & $\mathrm{~F}$ & & & & \\
\hline Guinea Marsh & $8 / 17 / 1999$ & Trans-brown & Intermediate & 604 & $\mathrm{~F}$ & 608.75 & & & \\
\hline Guinea Marsh & $8 / 17 / 1999$ & Trans-brown & Intermediate & 606 & $\mathrm{~F}$ & & & & \\
\hline Guinea Marsh & 8/17/1999 & Trans-brown & Intermediate & 610 & $\mathrm{~F}$ & & & & \\
\hline Guinea Marsh & 8/17/1999 & Transparent & New & 624 & $\mathrm{~F}$ & & & & \\
\hline Guinea Marsh & $8 / 17 / 1999$ & Transparent & New & 600 & $\mathrm{~F}$ & & & & \\
\hline Guinea Marsh & $8 / 17 / 1999$ & Transparent & New & 598 & $\mathrm{~F}$ & & & & \\
\hline Guinea Marsh & 8/17/1999 & Transparent & New & 610 & $\mathrm{~F}$ & & & & \\
\hline Guinea Marsh & 8/17/1999 & Very brown/opaque & Very old & 618 & $\mathrm{~F}$ & & & & \\
\hline Goodwin Island & 9/14/1999 & Brown & Old & 596 & $\mathrm{~F}$ & & 594.5 & & 594.8571429 \\
\hline Goodwin Island & $9 / 14 / 1999$ & Brown & Old & 594 & $\mathrm{~F}$ & & & & \\
\hline Goodwin Island & 9/14/1999 & Brown-opaque & Very old & 600 & $\mathrm{~F}$ & & & & \\
\hline Goodwin Island & 9/14/1999 & Brown-opaque & Very old & 602 & $\mathrm{~F}$ & & & & \\
\hline Goodwin Island & 9/14/1999 & Trans-brown & Intermediate & 590 & $\mathrm{~F}$ & & & & \\
\hline Goodwin Island & 9/14/1999 & Trans-brown & Intermediate & 570 & $\mathrm{~F}$ & & & & \\
\hline Goodwin Island & 9/14/1999 & Trans-brown & Intermediate & 596 & $\mathrm{~F}$ & & & & \\
\hline Goodwin Island & 9/14/1999 & Trans-brown & Intermediate & 608 & $\mathrm{~F}$ & & & & \\
\hline Guinea Marsh & 9/14/1999 & Brown & Old & 624 & $\mathrm{~F}$ & 595.0769231 & & & \\
\hline Guinea Marsh & 9/14/1999 & Brown & Old & 586 & $\mathrm{~F}$ & & & & \\
\hline Guinea Marsh & 9/14/1999 & Brown & Old & 596 & $\mathrm{~F}$ & & & & \\
\hline Guinea Marsh & 9/14/1999 & Brown & Old & 578 & $\mathrm{~F}$ & & & & \\
\hline Guinea Marsh & 9/14/1999 & Brown-opaque & Very old & 602 & $\mathrm{~F}$ & & & & \\
\hline Guinea Marsh & 9/14/1999 & Brown-opaque & Very old & 566 & $\mathrm{~F}$ & & & & \\
\hline Guinea Marsh & 9/14/1999 & Opaque-white & Very old & 608 & $\mathrm{~F}$ & & & & \\
\hline Guinea Marsh & 9/14/1999 & Trans-brown & Intermediate & 596 & $\mathrm{~F}$ & & & & \\
\hline Guinea Marsh & 9/14/1999 & Trans-brown & Intermediate & 576 & $\mathrm{~F}$ & & & & \\
\hline Guinea Marsh & 9/14/1999 & Trans-brown & Intermediate & 590 & $\mathrm{~F}$ & & & & \\
\hline Guinea Marsh & 9/14/1999 & Transparent & New & 610 & $\mathrm{~F}$ & & & & \\
\hline Guinea Marsh & $9 / 14 / 1999$ & Transparent & New & 600 & $\mathrm{~F}$ & & & & \\
\hline Guinea Marsh & 9/14/1999 & White-opaque & Very old & 604 & $\mathrm{~F}$ & & & & \\
\hline Guinea Marsh & $10 / 15 / 1999$ & Brown & Old & 604 & $\mathrm{~F}$ & 611.3333333 & & & 611.3333333 \\
\hline Guinea Marsh & $10 / 15 / 1999$ & Brown & Old & 602 & $\mathrm{~F}$ & & & & \\
\hline Guinea Marsh & $10 / 15 / 1999$ & Brown & Old & 600 & $\mathrm{~F}$ & & & & \\
\hline Guinea Marsh & $10 / 15 / 1999$ & Brown & Old & 596 & $\mathrm{~F}$ & & & & \\
\hline Guinea Marsh & $10 / 15 / 1999$ & Trans-brown & Intermediate & 608 & $\mathrm{~F}$ & & & & \\
\hline Guinea Marsh & $10 / 15 / 1999$ & Trans-brown & Intermediate & 588 & $\mathrm{~F}$ & & & & \\
\hline Guinea Marsh & $10 / 15 / 1999$ & Trans-brown & Intermediate & 624 & $\mathrm{~F}$ & & & & \\
\hline Guinea Marsh & $10 / 15 / 1999$ & Trans-brown & Intermediate & 622 & $\mathrm{~F}$ & & & & \\
\hline Guinea Marsh & $10 / 15 / 1999$ & Trans-brown & Intermediate & 640 & $\mathrm{~F}$ & & & & \\
\hline Guinea Marsh & $10 / 15 / 1999$ & Trans-brown & Intermediate & 618 & $\mathrm{~F}$ & & & & \\
\hline Guinea Marsh & $10 / 15 / 1999$ & Trans-brown & Intermediate & 600 & $\mathrm{~F}$ & & & & \\
\hline Guinea Marsh & $10 / 15 / 1999$ & Transparent & New & 620 & $\mathrm{~F}$ & & & & \\
\hline Guinea Marsh & $10 / 15 / 1999$ & Transparent & New & 610 & $\mathrm{~F}$ & & & & \\
\hline Guinea Marsh & $10 / 15 / 1999$ & Transparent & New & 622 & $\mathrm{~F}$ & & & & \\
\hline Guinea Marsh & $10 / 15 / 1999$ & Transparent & New & 622 & $\mathrm{~F}$ & & & & \\
\hline Guinea Marsh & $10 / 15 / 1999$ & Transparent & New & 624 & $\mathrm{~F}$ & & & & \\
\hline Guinea Marsh & $10 / 15 / 1999$ & Transparent & New & 618 & $\mathrm{~F}$ & & & & \\
\hline Guinea Marsh & $10 / 15 / 1999$ & Transparent & New & 628 & $\mathrm{~F}$ & & & & \\
\hline Guinea Marsh & $10 / 15 / 1999$ & Very brown/opaque & Very old & 610 & $\mathrm{~F}$ & & & & \\
\hline
\end{tabular}


Appendix A (continued)

\begin{tabular}{|c|c|c|c|c|c|c|c|c|c|}
\hline Site & Date & Color & Age & $\begin{array}{l}\text { Size } \\
(\mu \mathrm{m})\end{array}$ & Sex & $\begin{array}{l}\text { Mean size } \\
\text { Guinea }(\mu \mathrm{m})\end{array}$ & $\begin{array}{l}\text { Mean size } \\
\text { Goodwin }(\mu \mathrm{m})\end{array}$ & $\begin{array}{l}\text { Mean size } \\
\text { Dameron }(\mu \mathrm{m})\end{array}$ & $\begin{array}{l}\text { Grand mean } \\
\text { size }(\mu \mathrm{m})\end{array}$ \\
\hline Guinea Marsh & $10 / 15 / 1999$ & Very brown/opaque & Very old & 596 & $\mathrm{~F}$ & & & & \\
\hline Guinea Marsh & $10 / 15 / 1999$ & Very opaque & Very old & 586 & $\mathrm{~F}$ & & & & \\
\hline Goodwin Island & $11 / 9 / 1999$ & Brown & Old & 620 & F & & 612.8 & & 618.8888889 \\
\hline Goodwin Island & $11 / 9 / 1999$ & Brown & Old & 620 & $\mathrm{~F}$ & & & & \\
\hline Goodwin Island & $11 / 9 / 1999$ & Brown-white & Old & 620 & $\mathrm{~F}$ & & & & \\
\hline Goodwin Island & $11 / 9 / 1999$ & Trans-brown & Intermediate & 600 & F & & & & \\
\hline Goodwin Island & $11 / 9 / 1999$ & Trans-brown & Intermediate & 610 & $\mathrm{~F}$ & & & & \\
\hline Goodwin Island & $11 / 9 / 1999$ & Trans-brown & Intermediate & 606 & F & & & & \\
\hline Goodwin Island & $11 / 9 / 1999$ & Transparent & New & 624 & F & & & & \\
\hline Goodwin Island & $11 / 9 / 1999$ & Transparent & New & 600 & $\mathrm{~F}$ & & & & \\
\hline Goodwin Island & $11 / 9 / 1999$ & Transparent & New & 604 & $\mathrm{~F}$ & & & & \\
\hline Goodwin Island & $11 / 9 / 1999$ & Trans-white & Intermediate/old & 624 & $\mathrm{~F}$ & & & & \\
\hline Guinea Marsh & $11 / 9 / 1999$ & Brown & Old & 620 & F & 620.0377358 & & & \\
\hline Guinea Marsh & $11 / 9 / 1999$ & Brown-white & Old & 622 & $\mathrm{~F}$ & & & & \\
\hline Guinea Marsh & $11 / 9 / 1999$ & Brown-white & Old & 606 & $\mathrm{~F}$ & & & & \\
\hline Guinea Marsh & $11 / 9 / 1999$ & Trans-brown & Intermediate & 620 & F & & & & \\
\hline Guinea Marsh & 11/9/1999 & Trans-brown & Intermediate & 610 & F & & & & \\
\hline Guinea Marsh & $11 / 9 / 1999$ & Trans-brown & Intermediate & 620 & $\mathrm{~F}$ & & & & \\
\hline Guinea Marsh & 11/9/1999 & Trans-brown & Intermediate & 630 & F & & & & \\
\hline Guinea Marsh & $11 / 9 / 1999$ & Trans-brown & Intermediate & 620 & F & & & & \\
\hline Guinea Marsh & $11 / 9 / 1999$ & Trans-brown & Intermediate & 636 & F & & & & \\
\hline Guinea Marsh & 11/9/1999 & Trans-brown & Intermediate & 626 & F & & & & \\
\hline Guinea Marsh & $11 / 9 / 1999$ & Trans-brown & Intermediate & 624 & $\mathrm{~F}$ & & & & \\
\hline Guinea Marsh & $11 / 9 / 1999$ & Trans-brown & Intermediate & 610 & F & & & & \\
\hline Guinea Marsh & $11 / 9 / 1999$ & Trans-brown & Intermediate & 600 & F & & & & \\
\hline Guinea Marsh & $11 / 9 / 1999$ & Trans-brown & Intermediate & 630 & $\mathrm{~F}$ & & & & \\
\hline Guinea Marsh & $11 / 9 / 1999$ & Trans-brown & Intermediate & 620 & F & & & & \\
\hline Guinea Marsh & $11 / 9 / 1999$ & Trans-opaque & Intermediate & 620 & $\mathrm{~F}$ & & & & \\
\hline Guinea Marsh & $11 / 9 / 1999$ & Transparent & New & 622 & F & & & & \\
\hline Guinea Marsh & 11/9/1999 & Transparent & New & 640 & F & & & & \\
\hline Guinea Marsh & $11 / 9 / 1999$ & Transparent & New & 624 & F & & & & \\
\hline Guinea Marsh & $11 / 9 / 1999$ & Transparent & New & 630 & F & & & & \\
\hline Guinea Marsh & 11/9/1999 & Transparent & New & 620 & F & & & & \\
\hline Guinea Marsh & $11 / 9 / 1999$ & Transparent & New & 622 & $\mathrm{~F}$ & & & & \\
\hline Guinea Marsh & $11 / 9 / 1999$ & Transparent & New & 642 & F & & & & \\
\hline Guinea Marsh & 11/9/1999 & Transparent & New & 620 & F & & & & \\
\hline Guinea Marsh & $11 / 9 / 1999$ & Transparent & New & 626 & $\mathrm{~F}$ & & & & \\
\hline Guinea Marsh & 11/9/1999 & Transparent & New & 632 & F & & & & \\
\hline Guinea Marsh & $11 / 9 / 1999$ & Transparent & New & 624 & $\mathrm{~F}$ & & & & \\
\hline Guinea Marsh & $11 / 9 / 1999$ & Transparent & New & 630 & $\mathrm{~F}$ & & & & \\
\hline Guinea Marsh & 11/9/1999 & Transparent & New & 620 & F & & & & \\
\hline Guinea Marsh & $11 / 9 / 1999$ & Transparent & New & 620 & F & & & & \\
\hline Guinea Marsh & $11 / 9 / 1999$ & Transparent & New & 620 & F & & & & \\
\hline Guinea Marsh & 11/9/1999 & Transparent & New & 640 & F & & & & \\
\hline Guinea Marsh & $11 / 9 / 1999$ & Transparent & New & 630 & $\mathrm{~F}$ & & & & \\
\hline Guinea Marsh & $11 / 9 / 1999$ & Transparent & New & 620 & F & & & & \\
\hline Guinea Marsh & $11 / 9 / 1999$ & Transparent & New & 600 & F & & & & \\
\hline Guinea Marsh & $11 / 9 / 1999$ & Transparent & New & 610 & $\mathrm{~F}$ & & & & \\
\hline Guinea Marsh & $11 / 9 / 1999$ & Transparent & New & 624 & F & & & & \\
\hline Guinea Marsh & $11 / 9 / 1999$ & Transparent & New & 616 & F & & & & \\
\hline Guinea Marsh & $11 / 9 / 1999$ & Transparent & New & 640 & $\mathrm{~F}$ & & & & \\
\hline Guinea Marsh & $11 / 9 / 1999$ & Transparent & New & 612 & F & & & & \\
\hline Guinea Marsh & $11 / 9 / 1999$ & Transparent & New & 616 & $\mathrm{~F}$ & & & & \\
\hline Guinea Marsh & $11 / 9 / 1999$ & Transparent & New & 610 & $\mathrm{~F}$ & & & & \\
\hline
\end{tabular}


Appendix A (continued)

\begin{tabular}{|c|c|c|c|c|c|c|c|c|c|}
\hline Site & Date & Color & Age & $\begin{array}{l}\text { Size } \\
(\mu \mathrm{m})\end{array}$ & Sex & $\begin{array}{l}\text { Mean size } \\
\text { Guinea }(\mu \mathrm{m})\end{array}$ & $\begin{array}{l}\text { Mean size } \\
\text { Goodwin }(\mu \mathrm{m})\end{array}$ & $\begin{array}{l}\text { Mean size } \\
\text { Dameron }(\mu \mathrm{m})\end{array}$ & $\begin{array}{l}\text { Grand mean } \\
\text { size }(\mu \mathrm{m})\end{array}$ \\
\hline Guinea Marsh & $11 / 9 / 1999$ & Transparent & New & 620 & $\mathrm{~F}$ & & & & \\
\hline Guinea Marsh & 11/9/1999 & Transparent & New & 584 & $\mathrm{~F}$ & & & & \\
\hline Guinea Marsh & $11 / 9 / 1999$ & Transparent & New & 604 & $\mathrm{~F}$ & & & & \\
\hline Guinea Marsh & $11 / 9 / 1999$ & Transparent & New & 580 & $\mathrm{~F}$ & & & & \\
\hline Guinea Marsh & 11/9/1999 & Transparent & New & 620 & $\mathrm{~F}$ & & & & \\
\hline Guinea Marsh & $11 / 9 / 1999$ & Transparent & New & 640 & $\mathrm{~F}$ & & & & \\
\hline Guinea Marsh & 11/9/1999 & Transparent & New & 630 & $\mathrm{~F}$ & & & & \\
\hline Guinea Marsh & $11 / 9 / 1999$ & Transparent & New & 606 & $\mathrm{~F}$ & & & & \\
\hline Guinea Marsh & $11 / 9 / 1999$ & Transparent & New & 620 & $\mathrm{~F}$ & & & & \\
\hline Guinea Marsh & $11 / 9 / 1999$ & Transparent & New & 618 & F & & & & \\
\hline Guinea Marsh & $11 / 9 / 1999$ & Transparent & New & 616 & $\mathrm{~F}$ & & & & \\
\hline Goodwin Island & $12 / 21 / 1999$ & Brown & Old & 616 & $\mathrm{~F}$ & & 609.1818182 & & 609 \\
\hline Goodwin Island & $12 / 21 / 1999$ & Brown-white & Old & 610 & $\mathrm{~F}$ & & & & \\
\hline Goodwin Island & $12 / 21 / 1999$ & Brown-white & Old & 604 & $\mathrm{~F}$ & & & & \\
\hline Goodwin Island & $12 / 21 / 1999$ & Transparent & New & 596 & $\mathrm{~F}$ & & & & \\
\hline Goodwin Island & $12 / 21 / 1999$ & Transparent & New & 610 & $\mathrm{~F}$ & & & & \\
\hline Goodwin Island & $12 / 21 / 1999$ & Transparent & New & 606 & $\mathrm{~F}$ & & & & \\
\hline Goodwin Island & $12 / 21 / 1999$ & Transparent & New & 604 & $\mathrm{~F}$ & & & & \\
\hline Goodwin Island & $12 / 21 / 1999$ & Transparent & New & 620 & $\mathrm{~F}$ & & & & \\
\hline Goodwin Island & $12 / 21 / 1999$ & Transparent & New & 602 & $\mathrm{~F}$ & & & & \\
\hline Goodwin Island & $12 / 21 / 1999$ & Trans-brown & Intermediate & 616 & $\mathrm{~F}$ & & & & \\
\hline Goodwin Island & $12 / 21 / 1999$ & Trans-brown & Intermediate & 618 & $\mathrm{~F}$ & & & & \\
\hline Goodwin Island & $12 / 21 / 1999$ & Trans-brown & Intermediate & 626 & $\mathrm{~F}$ & & & & \\
\hline Goodwin Island & $12 / 21 / 1999$ & Trans-brown & Intermediate & 600 & $\mathrm{~F}$ & & & & \\
\hline Goodwin Island & $12 / 21 / 1999$ & Trans-brown & Intermediate & 604 & $\mathrm{~F}$ & & & & \\
\hline Goodwin Island & $12 / 21 / 1999$ & Trans-brown & Intermediate & 602 & $\mathrm{~F}$ & & & & \\
\hline Goodwin Island & $12 / 21 / 1999$ & Trans-brown & Intermediate & 600 & $\mathrm{~F}$ & & & & \\
\hline Goodwin Island & $12 / 21 / 1999$ & Transparent & New & 610 & $\mathrm{~F}$ & & & & \\
\hline Goodwin Island & $12 / 21 / 1999$ & Trans-white & Intermediate/old & 618 & $\mathrm{~F}$ & & & & \\
\hline Goodwin Island & $12 / 21 / 1999$ & White-opaque & Very old & 620 & $\mathrm{~F}$ & & & & \\
\hline Goodwin Island & $12 / 21 / 1999$ & White-opaque & Very old & 600 & $\mathrm{~F}$ & & & & \\
\hline Goodwin Island & $12 / 21 / 1999$ & White-opaque & Very old & 618 & $\mathrm{~F}$ & & & & \\
\hline Goodwin Island & $12 / 21 / 1999$ & White-opaque & Very old & 602 & $\mathrm{~F}$ & & & & \\
\hline Guinea Marsh & $12 / 21 / 1999$ & Brown & Old & 602 & $\mathrm{~F}$ & 608.9130435 & & & \\
\hline Guinea Marsh & $12 / 21 / 1999$ & Brown & Old & 600 & $\mathrm{~F}$ & & & & \\
\hline Guinea Marsh & $12 / 21 / 1999$ & Brown & Old & 580 & $\mathrm{~F}$ & & & & \\
\hline Guinea Marsh & $12 / 21 / 1999$ & Brown & Old & 620 & $\mathrm{~F}$ & & & & \\
\hline Guinea Marsh & $12 / 21 / 1999$ & Brown-white & Old & 600 & $\mathrm{~F}$ & & & & \\
\hline Guinea Marsh & $12 / 21 / 1999$ & Transparent & New & 606 & $\mathrm{~F}$ & & & & \\
\hline Guinea Marsh & $12 / 21 / 1999$ & Transparent & New & 620 & $\mathrm{~F}$ & & & & \\
\hline Guinea Marsh & $12 / 21 / 1999$ & Transparent & New & 614 & $\mathrm{~F}$ & & & & \\
\hline Guinea Marsh & $12 / 21 / 1999$ & Transparent & New & 608 & $\mathrm{~F}$ & & & & \\
\hline Guinea Marsh & $12 / 21 / 1999$ & Transparent & New & 618 & $\mathrm{~F}$ & & & & \\
\hline Guinea Marsh & $12 / 21 / 1999$ & Transparent & New & 600 & $\mathrm{~F}$ & & & & \\
\hline Guinea Marsh & $12 / 21 / 1999$ & Trans-white & Intermediate/old & 610 & $\mathrm{~F}$ & & & & \\
\hline Guinea Marsh & $12 / 21 / 1999$ & Trans-white & Intermediate/old & 580 & $\mathrm{~F}$ & & & & \\
\hline Guinea Marsh & $12 / 21 / 1999$ & Trans-white & Intermediate/old & 600 & $\mathrm{~F}$ & & & & \\
\hline Guinea Marsh & $12 / 21 / 1999$ & Trans-white & Intermediate/old & 630 & $\mathrm{~F}$ & & & & \\
\hline Guinea Marsh & $12 / 21 / 1999$ & Trans-white & Intermediate/old & 620 & $\mathrm{~F}$ & & & & \\
\hline Guinea Marsh & $12 / 21 / 1999$ & Trans-white & Intermediate/old & 620 & $\mathrm{~F}$ & & & & \\
\hline Guinea Marsh & $12 / 21 / 1999$ & Trans-white & Intermediate/old & 620 & $\mathrm{~F}$ & & & & \\
\hline Guinea Marsh & $12 / 21 / 1999$ & White-opaque & Very old & 584 & $\mathrm{~F}$ & & & & \\
\hline
\end{tabular}


Appendix A (continued)

\begin{tabular}{|c|c|c|c|c|c|c|c|c|c|}
\hline Site & Date & Color & Age & $\begin{array}{l}\text { Size } \\
(\mu \mathrm{m})\end{array}$ & Sex & $\begin{array}{l}\text { Mean size } \\
\text { Guinea }(\mu \mathrm{m})\end{array}$ & $\begin{array}{l}\text { Mean size } \\
\text { Goodwin }(\mu \mathrm{m})\end{array}$ & $\begin{array}{l}\text { Mean size } \\
\text { Dameron }(\mu \mathrm{m})\end{array}$ & $\begin{array}{l}\text { Grand mean } \\
\text { size }(\mu \mathrm{m})\end{array}$ \\
\hline Guinea Marsh & $12 / 21 / 1999$ & White-opaque & Very old & 610 & $\mathrm{~F}$ & & & & \\
\hline Guinea Marsh & 12/21/1999 & White-opaque & Very old & 600 & F & & & & \\
\hline Guinea Marsh & $12 / 21 / 1999$ & White-opaque & Very old & 610 & F & & & & \\
\hline Guinea Marsh & $12 / 21 / 1999$ & White-opaque & Very old & 600 & $\mathrm{~F}$ & & & & \\
\hline Guinea Marsh & 12/21/1999 & White-opaque & Very old & 638 & F & & & & \\
\hline Guinea Marsh & $12 / 21 / 1999$ & White-opaque & Very old & 626 & F & & & & \\
\hline Guinea Marsh & $12 / 21 / 1999$ & White-opaque & Very old & 620 & F & & & & \\
\hline Guinea Marsh & $12 / 21 / 1999$ & White-opaque & Very old & 624 & F & & & & \\
\hline Guinea Marsh & $12 / 21 / 1999$ & White-opaque & Very old & 622 & $\mathrm{~F}$ & & & & \\
\hline Guinea Marsh & $12 / 21 / 1999$ & White-opaque & Very old & 614 & F & & & & \\
\hline Guinea Marsh & $12 / 21 / 1999$ & White-opaque & Very old & 600 & F & & & & \\
\hline Guinea Marsh & $12 / 21 / 1999$ & White-opaque & Very old & 622 & $\mathrm{~F}$ & & & & \\
\hline Guinea Marsh & $12 / 21 / 1999$ & White-opaque & Very old & 620 & F & & & & \\
\hline Guinea Marsh & $12 / 21 / 1999$ & White-opaque & Very old & 606 & F & & & & \\
\hline Guinea Marsh & $12 / 21 / 1999$ & White-opaque & Very old & 590 & $\mathrm{~F}$ & & & & \\
\hline Guinea Marsh & $12 / 21 / 1999$ & White-opaque & Very old & 620 & F & & & & \\
\hline Guinea Marsh & $12 / 21 / 1999$ & White-opaque & Very old & 604 & F & & & & \\
\hline Guinea Marsh & $12 / 21 / 1999$ & White-opaque & Very old & 590 & $\mathrm{~F}$ & & & & \\
\hline Guinea Marsh & $12 / 21 / 1999$ & White-opaque & Very old & 640 & F & & & & \\
\hline Guinea Marsh & $12 / 21 / 1999$ & White-opaque & Very old & 600 & F & & & & \\
\hline Guinea Marsh & $12 / 21 / 1999$ & White-opaque & Very old & 620 & F & & & & \\
\hline Guinea Marsh & $12 / 21 / 1999$ & White-opaque & Very old & 580 & F & & & & \\
\hline Guinea Marsh & $12 / 21 / 1999$ & White-opaque & Very old & 622 & $\mathrm{~F}$ & & & & \\
\hline Guinea Marsh & $12 / 21 / 1999$ & White-opaque & Very old & 600 & F & & & & \\
\hline Guinea Marsh & $12 / 21 / 1999$ & White-opaque & Very old & 580 & $\mathrm{~F}$ & & & & \\
\hline Guinea Marsh & $12 / 21 / 1999$ & White-opaque & Very old & 640 & $\mathrm{~F}$ & & & & \\
\hline Guinea Marsh & $12 / 21 / 1999$ & White-opaque & Very old & 580 & F & & & & \\
\hline Goodwin Island & $1 / 8 / 2000$ & Brown & Old & 610 & $\mathrm{~F}$ & & 612.4583333 & & 610.2093023 \\
\hline Goodwin Island & $1 / 8 / 2000$ & Brown-white & Old & 584 & F & & & & \\
\hline Goodwin Island & $1 / 8 / 2000$ & Trans-brown & Intermediate & 604 & F & & & & \\
\hline Goodwin Island & $1 / 8 / 2000$ & Trans-brown & Intermediate & 620 & F & & & & \\
\hline Goodwin Island & $1 / 8 / 2000$ & Trans-brown & Intermediate & 608 & F & & & & \\
\hline Goodwin Island & $1 / 8 / 2000$ & Trans-brown & Intermediate & 630 & F & & & & \\
\hline Goodwin Island & $1 / 8 / 2000$ & Trans-brown & Intermediate & 610 & $\mathrm{~F}$ & & & & \\
\hline Goodwin Island & $1 / 8 / 2000$ & Trans-brown & Intermediate & 612 & F & & & & \\
\hline Goodwin Island & $1 / 8 / 2000$ & Trans-brown & Intermediate & 604 & F & & & & \\
\hline Goodwin Island & $1 / 8 / 2000$ & Trans-brown & Intermediate & 620 & $\mathrm{~F}$ & & & & \\
\hline Goodwin Island & $1 / 8 / 2000$ & Trans-brown & Intermediate & 620 & F & & & & \\
\hline Goodwin Island & $1 / 8 / 2000$ & Trans-brown & Intermediate & 618 & $\mathrm{~F}$ & & & & \\
\hline Goodwin Island & $1 / 8 / 2000$ & Trans-brown & Intermediate & 618 & $\mathrm{~F}$ & & & & \\
\hline Goodwin Island & $1 / 8 / 2000$ & Trans-brown & Intermediate & 600 & $\mathrm{~F}$ & & & & \\
\hline Goodwin Island & $1 / 8 / 2000$ & Trans-brown & Intermediate & 622 & $\mathrm{~F}$ & & & & \\
\hline Goodwin Island & $1 / 8 / 2000$ & Trans-brown & Intermediate & 616 & F & & & & \\
\hline Goodwin Island & $1 / 8 / 2000$ & Trans-brown & Intermediate & 604 & F & & & & \\
\hline Goodwin Island & $1 / 8 / 2000$ & Trans-brown & Intermediate & 606 & $\mathrm{~F}$ & & & & \\
\hline Goodwin Island & $1 / 8 / 2000$ & Trans-brown & Intermediate & 624 & F & & & & \\
\hline Goodwin Island & $1 / 8 / 2000$ & Trans-brown & Intermediate & 636 & F & & & & \\
\hline Goodwin Island & $1 / 8 / 2000$ & Trans-brown & Intermediate & 620 & $\mathrm{~F}$ & & & & \\
\hline Goodwin Island & $1 / 8 / 2000$ & Trans-brown & Intermediate & 630 & F & & & & \\
\hline Goodwin Island & $1 / 8 / 2000$ & Trans-brown & Intermediate & 624 & $\mathrm{~F}$ & & & & \\
\hline Goodwin Island & $1 / 8 / 2000$ & Trans-brown & Intermediate & 604 & $\mathrm{~F}$ & & & & \\
\hline Goodwin Island & $1 / 8 / 2000$ & Trans-brown & Intermediate & 596 & F & & & & \\
\hline Goodwin Island & $1 / 8 / 2000$ & Trans-brown & Intermediate & 600 & $\mathrm{~F}$ & & & & \\
\hline Goodwin Island & $1 / 8 / 2000$ & Trans-brown & Intermediate & 582 & $\mathrm{~F}$ & & & & \\
\hline
\end{tabular}


Appendix A (continued)

\begin{tabular}{|c|c|c|c|c|c|c|c|c|c|}
\hline Site & Date & Color & Age & $\begin{array}{l}\text { Size } \\
(\mu \mathrm{m})\end{array}$ & Sex & $\begin{array}{l}\text { Mean size } \\
\text { Guinea }(\mu \mathrm{m})\end{array}$ & $\begin{array}{l}\text { Mean size } \\
\text { Goodwin }(\mu \mathrm{m})\end{array}$ & $\begin{array}{l}\text { Mean size } \\
\text { Dameron }(\mu \mathrm{m})\end{array}$ & $\begin{array}{l}\text { Grand mean } \\
\text { size }(\mu \mathrm{m})\end{array}$ \\
\hline Goodwin Island & $1 / 8 / 2000$ & Trans-brown & Intermediate & 600 & $\mathrm{~F}$ & & & & \\
\hline Goodwin Island & $1 / 8 / 2000$ & Trans-brown & Intermediate & 604 & $\mathrm{~F}$ & & & & \\
\hline Goodwin Island & $1 / 8 / 2000$ & Trans-brown & Intermediate & 616 & $\mathrm{~F}$ & & & & \\
\hline Goodwin Island & $1 / 8 / 2000$ & Trans-brown & Intermediate & 630 & $\mathrm{~F}$ & & & & \\
\hline Goodwin Island & $1 / 8 / 2000$ & Trans-brown & Intermediate & 620 & $\mathrm{~F}$ & & & & \\
\hline Goodwin Island & $1 / 8 / 2000$ & Trans-brown & Intermediate & 600 & $\mathrm{~F}$ & & & & \\
\hline Goodwin Island & $1 / 8 / 2000$ & Trans-brown & Intermediate & 620 & $\mathrm{~F}$ & & & & \\
\hline Goodwin Island & $1 / 8 / 2000$ & Trans-brown & Intermediate & 610 & $\mathrm{~F}$ & & & & \\
\hline Goodwin Island & $1 / 8 / 2000$ & Transparent & New & 620 & $\mathrm{~F}$ & & & & \\
\hline Goodwin Island & $1 / 8 / 2000$ & Transparent & New & 618 & $\mathrm{~F}$ & & & & \\
\hline Goodwin Island & $1 / 8 / 2000$ & Transparent & New & 604 & $\mathrm{~F}$ & & & & \\
\hline Goodwin Island & $1 / 8 / 2000$ & Transparent & New & 600 & $\mathrm{~F}$ & & & & \\
\hline Goodwin Island & $1 / 8 / 2000$ & Transparent & New & 620 & $\mathrm{~F}$ & & & & \\
\hline Goodwin Island & $1 / 8 / 2000$ & Transparent & New & 632 & $\mathrm{~F}$ & & & & \\
\hline Goodwin Island & $1 / 8 / 2000$ & Transparent & New & 630 & $\mathrm{~F}$ & & & & \\
\hline Goodwin Island & $1 / 8 / 2000$ & Transparent & New & 640 & $\mathrm{~F}$ & & & & \\
\hline Goodwin Island & $1 / 8 / 2000$ & Transparent & New & 580 & $\mathrm{~F}$ & & & & \\
\hline Goodwin Island & $1 / 8 / 2000$ & Transparent & New & 606 & $\mathrm{~F}$ & & & & \\
\hline Goodwin Island & $1 / 8 / 2000$ & Transparent & New & 608 & $\mathrm{~F}$ & & & & \\
\hline Goodwin Island & $1 / 8 / 2000$ & Transparent & New & 618 & $\mathrm{~F}$ & & & & \\
\hline Goodwin Island & $1 / 8 / 2000$ & Very brown & Very old & 600 & $\mathrm{~F}$ & & & & \\
\hline Guinea Marsh & $1 / 8 / 2000$ & Brown & Old & 600 & $\mathrm{~F}$ & 607.3684211 & & & \\
\hline Guinea Marsh & $1 / 8 / 2000$ & Brown & Old & 620 & $\mathrm{~F}$ & & & & \\
\hline Guinea Marsh & $1 / 8 / 2000$ & Brown & Old & 622 & $\mathrm{~F}$ & & & & \\
\hline Guinea Marsh & $1 / 8 / 2000$ & Brown & Old & 604 & $\mathrm{~F}$ & & & & \\
\hline Guinea Marsh & $1 / 8 / 2000$ & Brown & Old & 612 & $\mathrm{~F}$ & & & & \\
\hline Guinea Marsh & $1 / 8 / 2000$ & Brown & Old & 604 & $\mathrm{~F}$ & & & & \\
\hline Guinea Marsh & $1 / 8 / 2000$ & Brown & Old & 600 & $\mathrm{~F}$ & & & & \\
\hline Guinea Marsh & $1 / 8 / 2000$ & Brown & Old & 604 & $\mathrm{~F}$ & & & & \\
\hline Guinea Marsh & $1 / 8 / 2000$ & Brown & Old & 628 & $\mathrm{~F}$ & & & & \\
\hline Guinea Marsh & $1 / 8 / 2000$ & Brown & Old & 618 & $\mathrm{~F}$ & & & & \\
\hline Guinea Marsh & $1 / 8 / 2000$ & Brown & Old & 596 & $\mathrm{~F}$ & & & & \\
\hline Guinea Marsh & $1 / 8 / 2000$ & Brown & Old & 600 & $\mathrm{~F}$ & & & & \\
\hline Guinea Marsh & $1 / 8 / 2000$ & Brown & Old & 624 & $\mathrm{~F}$ & & & & \\
\hline Guinea Marsh & $1 / 8 / 2000$ & Brown & Old & 620 & $\mathrm{~F}$ & & & & \\
\hline Guinea Marsh & $1 / 8 / 2000$ & Brown-white & Old & 604 & $\mathrm{~F}$ & & & & \\
\hline Guinea Marsh & $1 / 8 / 2000$ & Brown-white & Old & 586 & $\mathrm{~F}$ & & & & \\
\hline Guinea Marsh & $1 / 8 / 2000$ & Brown-white & Old & 602 & $\mathrm{~F}$ & & & & \\
\hline Guinea Marsh & $1 / 8 / 2000$ & Transparent & New & 612 & $\mathrm{~F}$ & & & & \\
\hline Guinea Marsh & $1 / 8 / 2000$ & Trans-brown & Intermediate & 620 & $\mathrm{~F}$ & & & & \\
\hline Guinea Marsh & $1 / 8 / 2000$ & Trans-brown & Intermediate & 600 & $\mathrm{~F}$ & & & & \\
\hline Guinea Marsh & $1 / 8 / 2000$ & Trans-brown & Intermediate & 600 & $\mathrm{~F}$ & & & & \\
\hline Guinea Marsh & $1 / 8 / 2000$ & Trans-brown & Intermediate & 612 & $\mathrm{~F}$ & & & & \\
\hline Guinea Marsh & $1 / 8 / 2000$ & Trans-brown & Intermediate & 600 & $\mathrm{~F}$ & & & & \\
\hline Guinea Marsh & $1 / 8 / 2000$ & Trans-brown & Intermediate & 600 & $\mathrm{~F}$ & & & & \\
\hline Guinea Marsh & $1 / 8 / 2000$ & Trans-brown & Intermediate & 600 & $\mathrm{~F}$ & & & & \\
\hline Guinea Marsh & $1 / 8 / 2000$ & Trans-brown & Intermediate & 606 & $\mathrm{~F}$ & & & & \\
\hline Guinea Marsh & $1 / 8 / 2000$ & Trans-brown & Intermediate & 618 & $\mathrm{~F}$ & & & & \\
\hline Guinea Marsh & $1 / 8 / 2000$ & Trans-brown & Intermediate & 600 & $\mathrm{~F}$ & & & & \\
\hline Guinea Marsh & $1 / 8 / 2000$ & Trans-brown & Intermediate & 600 & $\mathrm{~F}$ & & & & \\
\hline Guinea Marsh & $1 / 8 / 2000$ & Trans-brown & Intermediate & 596 & $\mathrm{~F}$ & & & & \\
\hline Guinea Marsh & $1 / 8 / 2000$ & Trans-brown & Intermediate & 618 & $\mathrm{~F}$ & & & & \\
\hline Guinea Marsh & $1 / 8 / 2000$ & Trans-brown & Intermediate & 616 & $\mathrm{~F}$ & & & & \\
\hline
\end{tabular}


Appendix A (continued)

\begin{tabular}{|c|c|c|c|c|c|c|c|c|c|}
\hline Site & Date & Color & Age & $\begin{array}{l}\text { Size } \\
(\mu \mathrm{m})\end{array}$ & Sex & $\begin{array}{l}\text { Mean size } \\
\text { Guinea }(\mu \mathrm{m})\end{array}$ & $\begin{array}{l}\text { Mean size } \\
\text { Goodwin }(\mu \mathrm{m})\end{array}$ & $\begin{array}{l}\text { Mean size } \\
\text { Dameron }(\mu \mathrm{m})\end{array}$ & $\begin{array}{l}\text { Grand mean } \\
\text { size }(\mu \mathrm{m})\end{array}$ \\
\hline Guinea Marsh & $1 / 8 / 2000$ & Trans-brown & Intermediate & 620 & $\mathrm{~F}$ & & & & \\
\hline Guinea Marsh & $1 / 8 / 2000$ & Transparent & New & 608 & F & & & & \\
\hline Guinea Marsh & $1 / 8 / 2000$ & Transparent & New & 600 & F & & & & \\
\hline Guinea Marsh & $1 / 8 / 2000$ & Transparent & New & 600 & F & & & & \\
\hline Guinea Marsh & $1 / 8 / 2000$ & Transparent & New & 620 & F & & & & \\
\hline Guinea Marsh & $1 / 8 / 2000$ & Very brown & Very old & 590 & F & & & & \\
\hline Guinea Marsh & $2 / 29 / 2000$ & Brown & Old & 620 & F & 611.0175439 & & & 611.76 \\
\hline Guinea Marsh & $2 / 29 / 2000$ & Brown & Old & 606 & F & & & & \\
\hline Guinea Marsh & $2 / 29 / 2000$ & Brown & Old & 600 & $\mathrm{~F}$ & & & & \\
\hline Guinea Marsh & $2 / 29 / 2000$ & Brown & Old & 600 & F & & & & \\
\hline Guinea Marsh & $2 / 29 / 2000$ & Brown & Old & 620 & F & & & & \\
\hline Guinea Marsh & $2 / 29 / 2000$ & Brown & Old & 640 & $\mathrm{~F}$ & & & & \\
\hline Guinea Marsh & $2 / 29 / 2000$ & Brown & Old & 640 & F & & & & \\
\hline Guinea Marsh & $2 / 29 / 2000$ & Brown & Old & 620 & F & & & & \\
\hline Guinea Marsh & $2 / 29 / 2000$ & Brown & Old & 618 & $\mathrm{~F}$ & & & & \\
\hline Guinea Marsh & $2 / 29 / 2000$ & Brown & Old & 616 & F & & & & \\
\hline Guinea Marsh & $2 / 29 / 2000$ & Brown & Old & 604 & $\mathrm{~F}$ & & & & \\
\hline Guinea Marsh & $2 / 29 / 2000$ & Brown & Old & 600 & F & & & & \\
\hline Guinea Marsh & $2 / 29 / 2000$ & Brown & Old & 560 & F & & & & \\
\hline Guinea Marsh & $2 / 29 / 2000$ & Brown & Old & 644 & $\mathrm{~F}$ & & & & \\
\hline Guinea Marsh & $2 / 29 / 2000$ & Brown & Old & 604 & F & & & & \\
\hline Guinea Marsh & $2 / 29 / 2000$ & Brown & Old & 620 & F & & & & \\
\hline Guinea Marsh & $2 / 29 / 2000$ & Brown & Old & 620 & $\mathrm{~F}$ & & & & \\
\hline Guinea Marsh & $2 / 29 / 2000$ & Brown & Old & 624 & F & & & & \\
\hline Guinea Marsh & $2 / 29 / 2000$ & Brown & Old & 604 & F & & & & \\
\hline Guinea Marsh & $2 / 29 / 2000$ & Brown & Old & 584 & F & & & & \\
\hline Guinea Marsh & $2 / 29 / 2000$ & Brown & Old & 602 & F & & & & \\
\hline Guinea Marsh & $2 / 29 / 2000$ & Brown & Old & 640 & F & & & & \\
\hline Guinea Marsh & $2 / 29 / 2000$ & Brown & Old & 636 & F & & & & \\
\hline Guinea Marsh & $2 / 29 / 2000$ & Brown & Old & 616 & F & & & & \\
\hline Guinea Marsh & $2 / 29 / 2000$ & Brown & Old & 600 & $\mathrm{~F}$ & & & & \\
\hline Guinea Marsh & $2 / 29 / 2000$ & Brown-white & Old & 630 & F & & & & \\
\hline Guinea Marsh & $2 / 29 / 2000$ & Brown-white & Old & 604 & F & & & & \\
\hline Guinea Marsh & $2 / 29 / 2000$ & Brown-white & Old & 624 & F & & & & \\
\hline Guinea Marsh & $2 / 29 / 2000$ & Brown-white & Old & 620 & F & & & & \\
\hline Guinea Marsh & $2 / 29 / 2000$ & Brown-white & Old & 618 & F & & & & \\
\hline Guinea Marsh & $2 / 29 / 2000$ & Brown-white & Old & 620 & F & & & & \\
\hline Guinea Marsh & $2 / 29 / 2000$ & Trans-brown & Intermediate & 620 & F & & & & \\
\hline Guinea Marsh & $2 / 29 / 2000$ & Trans-brown & Intermediate & 620 & $\mathrm{~F}$ & & & & \\
\hline Guinea Marsh & $2 / 29 / 2000$ & Trans-brown & Intermediate & 600 & F & & & & \\
\hline Guinea Marsh & $2 / 29 / 2000$ & Trans-brown & Intermediate & 594 & $\mathrm{~F}$ & & & & \\
\hline Guinea Marsh & $2 / 29 / 2000$ & Trans-brown & Intermediate & 600 & $\mathrm{~F}$ & & & & \\
\hline Guinea Marsh & $2 / 29 / 2000$ & Trans-brown & Intermediate & 608 & F & & & & \\
\hline Guinea Marsh & $2 / 29 / 2000$ & Trans-brown & Intermediate & 600 & F & & & & \\
\hline Guinea Marsh & $2 / 29 / 2000$ & Trans-brown & Intermediate & 604 & $\mathrm{~F}$ & & & & \\
\hline Guinea Marsh & $2 / 29 / 2000$ & Trans-brown & Intermediate & 614 & F & & & & \\
\hline Guinea Marsh & $2 / 29 / 2000$ & Trans-brown & Intermediate & 620 & F & & & & \\
\hline Guinea Marsh & $2 / 29 / 2000$ & Trans-opaque & Intermediate & 626 & F & & & & \\
\hline Guinea Marsh & $2 / 29 / 2000$ & Trans-opaque & Intermediate & 620 & F & & & & \\
\hline Guinea Marsh & $2 / 29 / 2000$ & Trans-white & Intermediate/old & 598 & $\mathrm{~F}$ & & & & \\
\hline Guinea Marsh & $2 / 29 / 2000$ & Trans-white & Intermediate/old & 600 & F & & & & \\
\hline Guinea Marsh & $2 / 29 / 2000$ & Very brown & Very old & 620 & F & & & & \\
\hline Guinea Marsh & $2 / 29 / 2000$ & Very brown & Very old & 580 & $\mathrm{~F}$ & & & & \\
\hline Guinea Marsh & $2 / 29 / 2000$ & Very brown & Very old & 596 & F & & & & \\
\hline
\end{tabular}


Appendix A (continued)

\begin{tabular}{|c|c|c|c|c|c|c|c|c|c|}
\hline Site & Date & Color & Age & $\begin{array}{l}\text { Size } \\
(\mu \mathrm{m})\end{array}$ & Sex & $\begin{array}{l}\text { Mean size } \\
\text { Guinea }(\mu \mathrm{m})\end{array}$ & $\begin{array}{l}\text { Mean size } \\
\text { Goodwin }(\mu \mathrm{m})\end{array}$ & $\begin{array}{l}\text { Mean size } \\
\text { Dameron }(\mu \mathrm{m})\end{array}$ & $\begin{array}{l}\text { Grand mean } \\
\text { size }(\mu \mathrm{m})\end{array}$ \\
\hline Guinea Marsh & $2 / 29 / 2000$ & Very brown & Very old & 614 & $\mathrm{~F}$ & & & & \\
\hline Guinea Marsh & $2 / 29 / 2000$ & Very brown & Very old & 600 & $\mathrm{~F}$ & & & & \\
\hline Guinea Marsh & $3 / 29 / 2000$ & Brown-white & Old & 620 & $\mathrm{~F}$ & & & & 605.7142857 \\
\hline Guinea Marsh & $3 / 29 / 2000$ & Brown-white & Old & 600 & $\mathrm{~F}$ & & & & \\
\hline Guinea Marsh & $3 / 29 / 2000$ & Brown-white & Old & 596 & $\mathrm{~F}$ & & & & \\
\hline Guinea Marsh & $3 / 29 / 2000$ & White-opaque & Very old & 604 & $\mathrm{~F}$ & & & & \\
\hline Guinea Marsh & $3 / 29 / 2000$ & White-opaque & Very old & 620 & $\mathrm{~F}$ & & & & \\
\hline Guinea Marsh & $3 / 29 / 2000$ & White-opaque & Very old & 600 & $\mathrm{~F}$ & & & & \\
\hline Guinea Marsh & $3 / 29 / 2000$ & White-opaque & Very old & 600 & $\mathrm{~F}$ & & & & \\
\hline Goodwin Island & $4 / 19 / 2000$ & Brown-white & Old & 616 & $\mathrm{~F}$ & & 605.3333333 & & 605.3333333 \\
\hline Goodwin Island & $4 / 19 / 2000$ & Brown-white & Old & 600 & $\mathrm{~F}$ & & & & \\
\hline Goodwin Island & $4 / 19 / 2000$ & Brown-white & Old & 600 & $\mathrm{~F}$ & & & & \\
\hline Dameron Marsh & $5 / 17 / 2000$ & Brown-white & Old & 606 & $\mathrm{~F}$ & & & 623.6 & 623.6 \\
\hline Dameron Marsh & $5 / 17 / 2000$ & Brown-white & Old & 624 & $\mathrm{~F}$ & & & & \\
\hline Dameron Marsh & $5 / 17 / 2000$ & Trans-brown & Intermediate & 614 & $\mathrm{~F}$ & & & & \\
\hline Dameron Marsh & $5 / 17 / 2000$ & Transparent & New & 636 & $\mathrm{~F}$ & & & & \\
\hline Dameron Marsh & $5 / 17 / 2000$ & Transparent & New & 638 & $\mathrm{~F}$ & & & & \\
\hline Goodwin Island & $6 / 13 / 2000$ & Brown & Old & 638 & $\mathrm{~F}$ & & 624.5454545 & & 624.4324324 \\
\hline Goodwin Island & $6 / 13 / 2000$ & Brown & Old & 640 & $\mathrm{~F}$ & & & & \\
\hline Goodwin Island & $6 / 13 / 2000$ & Brown & Old & 620 & $\mathrm{~F}$ & & & & \\
\hline Goodwin Island & $6 / 13 / 2000$ & Brown & Old & 624 & $\mathrm{~F}$ & & & & \\
\hline Goodwin Island & $6 / 13 / 2000$ & Brown & Old & 626 & $\mathrm{~F}$ & & & & \\
\hline Goodwin Island & $6 / 13 / 2000$ & Brown & Old & 626 & $\mathrm{~F}$ & & & & \\
\hline Goodwin Island & $6 / 13 / 2000$ & Brown & Old & 620 & $\mathrm{~F}$ & & & & \\
\hline Goodwin Island & $6 / 13 / 2000$ & Brown & Old & 630 & $\mathrm{~F}$ & & & & \\
\hline Goodwin Island & $6 / 13 / 2000$ & Transparent & New & 624 & $\mathrm{~F}$ & & & & \\
\hline Goodwin Island & $6 / 13 / 2000$ & Transparent & New & 616 & $\mathrm{~F}$ & & & & \\
\hline Goodwin Island & $6 / 13 / 2000$ & Transparent & New & 630 & $\mathrm{~F}$ & & & & \\
\hline Goodwin Island & $6 / 13 / 2000$ & Transparent & New & 642 & $\mathrm{~F}$ & & & & \\
\hline Goodwin Island & $6 / 13 / 2000$ & Transparent & New & 636 & $\mathrm{~F}$ & & & & \\
\hline Goodwin Island & $6 / 13 / 2000$ & Transparent & New & 624 & $\mathrm{~F}$ & & & & \\
\hline Goodwin Island & $6 / 13 / 2000$ & Transparent & New & 620 & $\mathrm{~F}$ & & & & \\
\hline Goodwin Island & $6 / 13 / 2000$ & Trans-brown & Intermediate & 624 & $\mathrm{~F}$ & & & & \\
\hline Goodwin Island & $6 / 13 / 2000$ & Trans-brown & Intermediate & 620 & $\mathrm{~F}$ & & & & \\
\hline Goodwin Island & $6 / 13 / 2000$ & Trans-brown & Intermediate & 624 & $\mathrm{~F}$ & & & & \\
\hline Goodwin Island & $6 / 13 / 2000$ & Trans-brown & Intermediate & 604 & $\mathrm{~F}$ & & & & \\
\hline Goodwin Island & $6 / 13 / 2000$ & Trans-brown & Intermediate & 624 & $\mathrm{~F}$ & & & & \\
\hline Goodwin Island & $6 / 13 / 2000$ & Trans-brown & Intermediate & 624 & $\mathrm{~F}$ & & & & \\
\hline Goodwin Island & $6 / 13 / 2000$ & Trans-brown & Intermediate & 634 & $\mathrm{~F}$ & & & & \\
\hline Goodwin Island & $6 / 13 / 2000$ & Trans-brown & Intermediate & 636 & $\mathrm{~F}$ & & & & \\
\hline Goodwin Island & $6 / 13 / 2000$ & Trans-brown & Intermediate & 638 & $\mathrm{~F}$ & & & & \\
\hline Goodwin Island & $6 / 13 / 2000$ & Trans-brown & Intermediate & 600 & $\mathrm{~F}$ & & & & \\
\hline Goodwin Island & $6 / 13 / 2000$ & Trans-brown & Intermediate & 640 & $\mathrm{~F}$ & & & & \\
\hline Goodwin Island & $6 / 13 / 2000$ & Trans-brown & Intermediate & 640 & $\mathrm{~F}$ & & & & \\
\hline Goodwin Island & $6 / 13 / 2000$ & Trans-brown & Intermediate & 600 & $\mathrm{~F}$ & & & & \\
\hline Goodwin Island & $6 / 13 / 2000$ & Very brown & Very old & 624 & $\mathrm{~F}$ & & & & \\
\hline Goodwin Island & $6 / 13 / 2000$ & White-opaque & Very old & 626 & $\mathrm{~F}$ & & & & \\
\hline Goodwin Island & $6 / 13 / 2000$ & White-opaque & Very old & 624 & $\mathrm{~F}$ & & & & \\
\hline Goodwin Island & $6 / 13 / 2000$ & White-opaque & Very old & 612 & $\mathrm{~F}$ & & & & \\
\hline Goodwin Island & $6 / 13 / 2000$ & White-opaque & Very old & 600 & $\mathrm{~F}$ & & & & \\
\hline Guinea Marsh & $6 / 13 / 2000$ & Trans-brown & Intermediate & 620 & $\mathrm{~F}$ & 623.5 & & & \\
\hline Guinea Marsh & $6 / 13 / 2000$ & Trans-brown & Intermediate & 620 & $\mathrm{~F}$ & & & & \\
\hline Guinea Marsh & $6 / 13 / 2000$ & Transparent & New & 626 & $\mathrm{~F}$ & & & & \\
\hline
\end{tabular}


Appendix A (continued)

\begin{tabular}{|c|c|c|c|c|c|c|c|c|c|}
\hline Site & Date & Color & Age & $\begin{array}{l}\text { Size } \\
(\mu \mathrm{m})\end{array}$ & Sex & $\begin{array}{l}\text { Mean size } \\
\text { Guinea }(\mu \mathrm{m})\end{array}$ & $\begin{array}{l}\text { Mean size } \\
\text { Goodwin }(\mu \mathrm{m})\end{array}$ & $\begin{array}{l}\text { Mean size } \\
\text { Dameron }(\mu \mathrm{m})\end{array}$ & $\begin{array}{l}\text { Grand mean } \\
\text { size }(\mu \mathrm{m})\end{array}$ \\
\hline Guinea Marsh & $6 / 13 / 2000$ & Transparent & New & 628 & $\mathrm{~F}$ & & & & \\
\hline Goodwin Island & $7 / 11 / 2000$ & Brown & Old & 602 & F & & 616.6315789 & & 620.3243243 \\
\hline Goodwin Island & $7 / 11 / 2000$ & Brown & Old & 624 & F & & & & \\
\hline Goodwin Island & $7 / 11 / 2000$ & Brown & Old & 622 & F & & & & \\
\hline Goodwin Island & $7 / 11 / 2000$ & Brown & Old & 620 & F & & & & \\
\hline Goodwin Island & $7 / 11 / 2000$ & Brown-white & Old & 600 & F & & & & \\
\hline Goodwin Island & $7 / 11 / 2000$ & Brown-white & Old & 620 & F & & & & \\
\hline Goodwin Island & $7 / 11 / 2000$ & Brown-white & Old & 620 & F & & & & \\
\hline Goodwin Island & $7 / 11 / 2000$ & Brown-white & Old & 604 & $\mathrm{~F}$ & & & & \\
\hline Goodwin Island & $7 / 11 / 2000$ & Brown-white & Old & 640 & F & & & & \\
\hline Goodwin Island & $7 / 11 / 2000$ & Transparent & New & 608 & F & & & & \\
\hline Goodwin Island & $7 / 11 / 2000$ & Transparent & New & 630 & $\mathrm{~F}$ & & & & \\
\hline Goodwin Island & $7 / 11 / 2000$ & Trans-brown & Intermediate & 614 & F & & & & \\
\hline Goodwin Island & $7 / 11 / 2000$ & Trans-brown & Intermediate & 610 & F & & & & \\
\hline Goodwin Island & $7 / 11 / 2000$ & Trans-brown & Intermediate & 604 & $\mathrm{~F}$ & & & & \\
\hline Goodwin Island & $7 / 11 / 2000$ & Trans-white & Intermediate/old & 632 & F & & & & \\
\hline Goodwin Island & $7 / 11 / 2000$ & Very brown & Very old & 618 & $\mathrm{~F}$ & & & & \\
\hline Goodwin Island & $7 / 11 / 2000$ & White-opaque & Very old & 622 & F & & & & \\
\hline Goodwin Island & $7 / 11 / 2000$ & White-opaque & Very old & 620 & F & & & & \\
\hline Goodwin Island & $7 / 11 / 2000$ & White-opaque & Very old & 606 & $\mathrm{~F}$ & & & & \\
\hline Guinea Marsh & $7 / 11 / 2000$ & Brown & old & 640 & F & 624.2222222 & & & \\
\hline Guinea Marsh & $7 / 11 / 2000$ & Brown-white & Old & 622 & F & & & & \\
\hline Guinea Marsh & $7 / 11 / 2000$ & Transparent & New & 610 & $\mathrm{~F}$ & & & & \\
\hline Guinea Marsh & $7 / 11 / 2000$ & Transparent & New & 620 & F & & & & \\
\hline Guinea Marsh & $7 / 11 / 2000$ & Transparent & New & 614 & F & & & & \\
\hline Guinea Marsh & $7 / 11 / 2000$ & Transparent & New & 620 & F & & & & \\
\hline Guinea Marsh & $7 / 11 / 2000$ & Transparent & New & 620 & F & & & & \\
\hline Guinea Marsh & $7 / 11 / 2000$ & Transparent & New & 640 & F & & & & \\
\hline Guinea Marsh & $7 / 11 / 2000$ & Transparent & New & 636 & F & & & & \\
\hline Guinea Marsh & $7 / 11 / 2000$ & Transparent & New & 606 & F & & & & \\
\hline Guinea Marsh & $7 / 11 / 2000$ & White-opaque & Very old & 604 & F & & & & \\
\hline Guinea Marsh & $7 / 11 / 2000$ & White-opaque & Very old & 640 & F & & & & \\
\hline Guinea Marsh & $7 / 11 / 2000$ & White-opaque & Very old & 640 & F & & & & \\
\hline Guinea Marsh & $7 / 11 / 2000$ & White-opaque & Very old & 642 & F & & & & \\
\hline Guinea Marsh & $7 / 11 / 2000$ & White-opaque & Very old & 634 & F & & & & \\
\hline Guinea Marsh & $7 / 11 / 2000$ & White-opaque & Very old & 624 & F & & & & \\
\hline Guinea Marsh & $7 / 11 / 2000$ & White-opaque & Very old & 600 & F & & & & \\
\hline Guinea Marsh & $7 / 11 / 2000$ & White-opaque & Very old & 624 & F & & & & \\
\hline Goodwin Island & $8 / 1 / 2000$ & Brown & old & 600 & F & & 613.4545455 & & 617.04 \\
\hline Goodwin Island & $8 / 1 / 2000$ & Brown-white & Old & 626 & F & & & & \\
\hline Goodwin Island & $8 / 1 / 2000$ & Trans-brown & Intermediate & 616 & F & & & & \\
\hline Goodwin Island & $8 / 1 / 2000$ & Trans-white & Intermediate/old & 600 & $\mathrm{~F}$ & & & & \\
\hline Goodwin Island & $8 / 1 / 2000$ & Trans-white & Intermediate/old & 620 & F & & & & \\
\hline Goodwin Island & $8 / 1 / 2000$ & White-opaque & Very old & 620 & F & & & & \\
\hline Goodwin Island & $8 / 1 / 2000$ & White-opaque & Very old & 614 & $\mathrm{~F}$ & & & & \\
\hline Goodwin Island & $8 / 1 / 2000$ & White-opaque & Very old & 584 & F & & & & \\
\hline Goodwin Island & $8 / 1 / 2000$ & White-opaque & Very old & 614 & F & & & & \\
\hline Goodwin Island & $8 / 1 / 2000$ & White-opaque & Very old & 640 & F & & & & \\
\hline Goodwin Island & $8 / 1 / 2000$ & White-opaque & Very old & 614 & F & & & & \\
\hline Guinea Marsh & $8 / 1 / 2000$ & Brown & Old & 600 & $\mathrm{~F}$ & 619.8571429 & & & \\
\hline Guinea Marsh & $8 / 1 / 2000$ & Brown & Old & 620 & F & & & & \\
\hline Guinea Marsh & $8 / 1 / 2000$ & Brown & Old & 608 & F & & & & \\
\hline Guinea Marsh & $8 / 1 / 2000$ & Brown & Old & 624 & $\mathrm{~F}$ & & & & \\
\hline Guinea Marsh & $8 / 1 / 2000$ & Brown-white & Old & 620 & $\mathrm{~F}$ & & & & \\
\hline
\end{tabular}


Appendix A (continued)

\begin{tabular}{|c|c|c|c|c|c|c|c|c|c|}
\hline Site & Date & Color & Age & $\begin{array}{l}\text { Size } \\
(\mu \mathrm{m})\end{array}$ & Sex & $\begin{array}{l}\text { Mean size } \\
\text { Guinea }(\mu \mathrm{m})\end{array}$ & $\begin{array}{l}\text { Mean size } \\
\text { Goodwin }(\mu \mathrm{m})\end{array}$ & $\begin{array}{l}\text { Mean size } \\
\text { Dameron }(\mu \mathrm{m})\end{array}$ & $\begin{array}{l}\text { Grand mean } \\
\text { size }(\mu \mathrm{m})\end{array}$ \\
\hline Guinea Marsh & $8 / 1 / 2000$ & Brown-white & Old & 624 & $\mathrm{~F}$ & & & & \\
\hline Guinea Marsh & $8 / 1 / 2000$ & Brown-white & Old & 600 & $\mathrm{~F}$ & & & & \\
\hline Guinea Marsh & $8 / 1 / 2000$ & Brown-white & Old & 600 & $\mathrm{~F}$ & & & & \\
\hline Guinea Marsh & $8 / 1 / 2000$ & Brown-white & Old & 616 & $\mathrm{~F}$ & & & & \\
\hline Guinea Marsh & $8 / 1 / 2000$ & Trans-white & Intermediate/old & 630 & $\mathrm{~F}$ & & & & \\
\hline Guinea Marsh & $8 / 1 / 2000$ & Trans-white & Intermediate/old & 624 & $\mathrm{~F}$ & & & & \\
\hline Guinea Marsh & $8 / 1 / 2000$ & Very brown & Very old & 608 & $\mathrm{~F}$ & & & & \\
\hline Guinea Marsh & $8 / 1 / 2000$ & White-opaque & Very old & 624 & $\mathrm{~F}$ & & & & \\
\hline Goodwin Island & $8 / 22 / 2000$ & Brown & Old & 620 & $\mathrm{~F}$ & & 621.0769231 & & 616.6153846 \\
\hline Goodwin Island & $8 / 22 / 2000$ & Brown-white & Old & 634 & $\mathrm{~F}$ & & & & \\
\hline Goodwin Island & $8 / 22 / 2000$ & Brown-white & Old & 624 & $\mathrm{~F}$ & & & & \\
\hline Goodwin Island & $8 / 22 / 2000$ & Brown-white & Old & 630 & $\mathrm{~F}$ & & & & \\
\hline Goodwin Island & $8 / 22 / 2000$ & Trans-brown & Intermediate & 642 & $\mathrm{~F}$ & & & & \\
\hline Goodwin Island & $8 / 22 / 2000$ & Trans-brown & Intermediate & 600 & $\mathrm{~F}$ & & & & \\
\hline Goodwin Island & $8 / 22 / 2000$ & Trans-brown & Intermediate & 620 & $\mathrm{~F}$ & & & & \\
\hline Goodwin Island & $8 / 22 / 2000$ & Trans-brown & Intermediate & 620 & $\mathrm{~F}$ & & & & \\
\hline Goodwin Island & $8 / 22 / 2000$ & Trans-brown & Intermediate & 640 & $\mathrm{~F}$ & & & & \\
\hline Goodwin Island & $8 / 22 / 2000$ & Trans-brown & Intermediate & 596 & $\mathrm{~F}$ & & & & \\
\hline Goodwin Island & $8 / 22 / 2000$ & Trans-brown & Intermediate & 626 & $\mathrm{~F}$ & & & & \\
\hline Goodwin Island & $8 / 22 / 2000$ & Trans-brown & Intermediate & 642 & $\mathrm{~F}$ & & & & \\
\hline Goodwin Island & $8 / 22 / 2000$ & Trans-brown & Intermediate & 620 & $\mathrm{~F}$ & & & & \\
\hline Goodwin Island & $8 / 22 / 2000$ & Transparent & New & 640 & $\mathrm{~F}$ & & & & \\
\hline Goodwin Island & $8 / 22 / 2000$ & Transparent & New & 604 & $\mathrm{~F}$ & & & & \\
\hline Goodwin Island & $8 / 22 / 2000$ & Transparent & New & 612 & $\mathrm{~F}$ & & & & \\
\hline Goodwin Island & $8 / 22 / 2000$ & Transparent & New & 608 & $\mathrm{~F}$ & & & & \\
\hline Goodwin Island & $8 / 22 / 2000$ & Transparent & New & 600 & $\mathrm{~F}$ & & & & \\
\hline Goodwin Island & $8 / 22 / 2000$ & Transparent & New & 620 & $\mathrm{~F}$ & & & & \\
\hline Goodwin Island & $8 / 22 / 2000$ & Transparent & New & 600 & $\mathrm{~F}$ & & & & \\
\hline Goodwin Island & $8 / 22 / 2000$ & White-opaque & Very old & 640 & $\mathrm{~F}$ & & & & \\
\hline Goodwin Island & $8 / 22 / 2000$ & White-opaque & Very old & 624 & $\mathrm{~F}$ & & & & \\
\hline Goodwin Island & $8 / 22 / 2000$ & White-opaque & Very old & 620 & $\mathrm{~F}$ & & & & \\
\hline Goodwin Island & $8 / 22 / 2000$ & White-opaque & Very old & 624 & $\mathrm{~F}$ & & & & \\
\hline Goodwin Island & $8 / 22 / 2000$ & White-opaque & Very old & 620 & $\mathrm{~F}$ & & & & \\
\hline Goodwin Island & $8 / 22 / 2000$ & White-opaque & Very old & 622 & $\mathrm{~F}$ & & & & \\
\hline Guinea Marsh & $8 / 22 / 2000$ & Brown & Old & 604 & $\mathrm{~F}$ & 612.1538462 & & & \\
\hline Guinea Marsh & $8 / 22 / 2000$ & Brown & Old & 626 & $\mathrm{~F}$ & & & & \\
\hline Guinea Marsh & $8 / 22 / 2000$ & Brown & Old & 604 & $\mathrm{~F}$ & & & & \\
\hline Guinea Marsh & $8 / 22 / 2000$ & Brown & Old & 618 & $\mathrm{~F}$ & & & & \\
\hline Guinea Marsh & $8 / 22 / 2000$ & Brown & Old & 604 & $\mathrm{~F}$ & & & & \\
\hline Guinea Marsh & $8 / 22 / 2000$ & Trans-brown & Intermediate & 624 & $\mathrm{~F}$ & & & & \\
\hline Guinea Marsh & $8 / 22 / 2000$ & Trans-brown & Intermediate & 580 & $\mathrm{~F}$ & & & & \\
\hline Guinea Marsh & $8 / 22 / 2000$ & Trans-brown & Intermediate & 600 & $\mathrm{~F}$ & & & & \\
\hline Guinea Marsh & $8 / 22 / 2000$ & Trans-brown & Intermediate & 596 & $\mathrm{~F}$ & & & & \\
\hline Guinea Marsh & $8 / 22 / 2000$ & Trans-brown & Intermediate & 604 & $\mathrm{~F}$ & & & & \\
\hline Guinea Marsh & $8 / 22 / 2000$ & Trans-brown & Intermediate & 580 & $\mathrm{~F}$ & & & & \\
\hline Guinea Marsh & $8 / 22 / 2000$ & Trans-brown & Intermediate & 620 & $\mathrm{~F}$ & & & & \\
\hline Guinea Marsh & $8 / 22 / 2000$ & Trans-brown & Intermediate & 620 & $\mathrm{~F}$ & & & & \\
\hline Guinea Marsh & $8 / 22 / 2000$ & Trans-brown & Intermediate & 624 & $\mathrm{~F}$ & & & & \\
\hline Guinea Marsh & $8 / 22 / 2000$ & Trans-brown & Intermediate & 626 & $\mathrm{~F}$ & & & & \\
\hline Guinea Marsh & $8 / 22 / 2000$ & Trans-brown & Intermediate & 604 & $\mathrm{~F}$ & & & & \\
\hline Guinea Marsh & $8 / 22 / 2000$ & Transparent & New & 600 & $\mathrm{~F}$ & & & & \\
\hline Guinea Marsh & $8 / 22 / 2000$ & Transparent & New & 600 & $\mathrm{~F}$ & & & & \\
\hline Guinea Marsh & $8 / 22 / 2000$ & Transparent & New & 604 & $\mathrm{~F}$ & & & & \\
\hline
\end{tabular}


Appendix A (continued)

\begin{tabular}{|c|c|c|c|c|c|c|c|c|c|}
\hline Site & Date & Color & Age & $\begin{array}{l}\text { Size } \\
(\mu \mathrm{m})\end{array}$ & Sex & $\begin{array}{l}\text { Mean size } \\
\text { Guinea }(\mu \mathrm{m})\end{array}$ & $\begin{array}{l}\text { Mean size } \\
\text { Goodwin }(\mu \mathrm{m})\end{array}$ & $\begin{array}{l}\text { Mean size } \\
\text { Dameron }(\mu \mathrm{m})\end{array}$ & $\begin{array}{l}\text { Grand mean } \\
\text { size }(\mu \mathrm{m})\end{array}$ \\
\hline Guinea Marsh & $8 / 22 / 2000$ & Transparent & New & 620 & $\mathrm{~F}$ & & & & \\
\hline Guinea Marsh & $8 / 22 / 2000$ & Transparent & New & 616 & F & & & & \\
\hline Guinea Marsh & $8 / 22 / 2000$ & Transparent & New & 622 & F & & & & \\
\hline Guinea Marsh & $8 / 22 / 2000$ & Transparent & New & 616 & $\mathrm{~F}$ & & & & \\
\hline Guinea Marsh & $8 / 22 / 2000$ & Transparent & New & 640 & F & & & & \\
\hline Guinea Marsh & $8 / 22 / 2000$ & Transparent & New & 642 & F & & & & \\
\hline Guinea Marsh & $8 / 22 / 2000$ & Transparent & New & 622 & F & & & & \\
\hline Goodwin Island & $9 / 6 / 2000$ & Brown-white & Old & 620 & F & & 622.8 & & 617.6428571 \\
\hline Goodwin Island & $9 / 6 / 2000$ & White-opaque & Very old & 640 & $\mathrm{~F}$ & & & & \\
\hline Goodwin Island & $9 / 6 / 2000$ & White-opaque & Very old & 600 & F & & & & \\
\hline Goodwin Island & $9 / 6 / 2000$ & White-opaque & Very old & 634 & F & & & & \\
\hline Goodwin Island & $9 / 6 / 2000$ & White-opaque & Very old & 620 & $\mathrm{~F}$ & & & & \\
\hline Guinea Marsh & $9 / 6 / 2000$ & Brown & Old & 616 & F & 616.5217391 & & & \\
\hline Guinea Marsh & $9 / 6 / 2000$ & Brown & Old & 620 & F & & & & \\
\hline Guinea Marsh & $9 / 6 / 2000$ & Brown-white & Old & 620 & $\mathrm{~F}$ & & & & \\
\hline Guinea Marsh & $9 / 6 / 2000$ & Brown-white & Old & 580 & F & & & & \\
\hline Guinea Marsh & $9 / 6 / 2000$ & Brown-white & Old & 624 & $\mathrm{~F}$ & & & & \\
\hline Guinea Marsh & $9 / 6 / 2000$ & Brown-white & Old & 640 & $\mathrm{~F}$ & & & & \\
\hline Guinea Marsh & $9 / 6 / 2000$ & Brown-white & Old & 600 & F & & & & \\
\hline Guinea Marsh & $9 / 6 / 2000$ & Trans-brown & Intermediate & 624 & $\mathrm{~F}$ & & & & \\
\hline Guinea Marsh & $9 / 6 / 2000$ & Trans-brown & Intermediate & 640 & F & & & & \\
\hline Guinea Marsh & $9 / 6 / 2000$ & Trans-brown & Intermediate & 638 & F & & & & \\
\hline Guinea Marsh & $9 / 6 / 2000$ & Trans-brown & Intermediate & 620 & $\mathrm{~F}$ & & & & \\
\hline Guinea Marsh & $9 / 6 / 2000$ & Trans-brown & Intermediate & 620 & F & & & & \\
\hline Guinea Marsh & $9 / 6 / 2000$ & Trans-brown & Intermediate & 610 & F & & & & \\
\hline Guinea Marsh & $9 / 6 / 2000$ & Trans-brown & Intermediate & 650 & $\mathrm{~F}$ & & & & \\
\hline Guinea Marsh & $9 / 6 / 2000$ & Transparent & New & 620 & F & & & & \\
\hline Guinea Marsh & $9 / 6 / 2000$ & Transparent & New & 636 & $\mathrm{~F}$ & & & & \\
\hline Guinea Marsh & $9 / 6 / 2000$ & Transparent & New & 608 & F & & & & \\
\hline Guinea Marsh & $9 / 6 / 2000$ & Transparent & New & 600 & F & & & & \\
\hline Guinea Marsh & $9 / 6 / 2000$ & White-opaque & Very old & 594 & F & & & & \\
\hline Guinea Marsh & $9 / 6 / 2000$ & White-opaque & Very old & 604 & F & & & & \\
\hline Guinea Marsh & $9 / 6 / 2000$ & White-opaque & Very old & 620 & F & & & & \\
\hline Guinea Marsh & $9 / 6 / 2000$ & White-opaque & Very old & 580 & $\mathrm{~F}$ & & & & \\
\hline Guinea Marsh & $9 / 6 / 2000$ & White-opaque & Very old & 616 & F & & & & \\
\hline Goodwin Island & $9 / 20 / 2000$ & Trans-brown & Intermediate & 604 & F & & 612 & & 611.7142857 \\
\hline Goodwin Island & $9 / 20 / 2000$ & Trans-brown & Intermediate & 616 & $\mathrm{~F}$ & & & & \\
\hline Goodwin Island & $9 / 20 / 2000$ & Trans-brown & Intermediate & 620 & F & & & & \\
\hline Goodwin Island & $9 / 20 / 2000$ & Trans-brown & Intermediate & 600 & F & & & & \\
\hline Goodwin Island & $9 / 20 / 2000$ & Trans-brown & Intermediate & 620 & $\mathrm{~F}$ & & & & \\
\hline Goodwin Island & $9 / 20 / 2000$ & Trans-brown & Intermediate & 620 & F & & & & \\
\hline Goodwin Island & $9 / 20 / 2000$ & Transparent & New & 604 & F & & & & \\
\hline Guinea Marsh & $9 / 20 / 2000$ & Brown & Old & 582 & F & 611.6190476 & & & \\
\hline Guinea Marsh & $9 / 20 / 2000$ & Trans-brown & Intermediate & 618 & F & & & & \\
\hline Guinea Marsh & $9 / 20 / 2000$ & Trans-brown & Intermediate & 600 & $\mathrm{~F}$ & & & & \\
\hline Guinea Marsh & $9 / 20 / 2000$ & Trans-brown & Intermediate & 600 & F & & & & \\
\hline Guinea Marsh & $9 / 20 / 2000$ & Trans-brown & Intermediate & 620 & F & & & & \\
\hline Guinea Marsh & $9 / 20 / 2000$ & Trans-brown & Intermediate & 620 & $\mathrm{~F}$ & & & & \\
\hline Guinea Marsh & $9 / 20 / 2000$ & Trans-brown & Intermediate & 618 & F & & & & \\
\hline Guinea Marsh & $9 / 20 / 2000$ & Trans-brown & Intermediate & 624 & F & & & & \\
\hline Guinea Marsh & $9 / 20 / 2000$ & Trans-brown & Intermediate & 604 & $\mathrm{~F}$ & & & & \\
\hline Guinea Marsh & $9 / 20 / 2000$ & Trans-brown & Intermediate & 624 & F & & & & \\
\hline Guinea Marsh & $9 / 20 / 2000$ & Trans-brown & Intermediate & 620 & $\mathrm{~F}$ & & & & \\
\hline Guinea Marsh & $9 / 20 / 2000$ & Trans-brown & Intermediate & 590 & $\mathrm{~F}$ & & & & \\
\hline
\end{tabular}


Appendix A (continued)

\begin{tabular}{|c|c|c|c|c|c|c|c|c|c|}
\hline Site & Date & Color & Age & $\begin{array}{l}\text { Size } \\
(\mu \mathrm{m})\end{array}$ & Sex & $\begin{array}{l}\text { Mean size } \\
\text { Guinea }(\mu \mathrm{m})\end{array}$ & $\begin{array}{l}\text { Mean size } \\
\text { Goodwin }(\mu \mathrm{m})\end{array}$ & $\begin{array}{l}\text { Mean size } \\
\text { Dameron }(\mu \mathrm{m})\end{array}$ & $\begin{array}{l}\text { Grand mean } \\
\text { size }(\mu \mathrm{m})\end{array}$ \\
\hline Guinea Marsh & $9 / 20 / 2000$ & Trans-brown & Intermediate & 598 & $\mathrm{~F}$ & & & & \\
\hline Guinea Marsh & $9 / 20 / 2000$ & Trans-brown & Intermediate & 580 & F & & & & \\
\hline Guinea Marsh & $9 / 20 / 2000$ & Transparent & New & 584 & $\mathrm{~F}$ & & & & \\
\hline Guinea Marsh & $9 / 20 / 2000$ & Transparent & New & 604 & $\mathrm{~F}$ & & & & \\
\hline Guinea Marsh & $9 / 20 / 2000$ & Transparent & New & 638 & $\mathrm{~F}$ & & & & \\
\hline Guinea Marsh & $9 / 20 / 2000$ & Transparent & New & 640 & $\mathrm{~F}$ & & & & \\
\hline Guinea Marsh & $9 / 20 / 2000$ & Transparent & New & 632 & $\mathrm{~F}$ & & & & \\
\hline Guinea Marsh & $9 / 20 / 2000$ & Transparent & New & 638 & $\mathrm{~F}$ & & & & \\
\hline Guinea Marsh & $9 / 20 / 2000$ & White-opaque & Very old & 610 & $\mathrm{~F}$ & & & & \\
\hline Goodwin Island & $10 / 3 / 2000$ & Trans-brown & Intermediate & 600 & F & & 616.8571429 & & 617.3513514 \\
\hline Goodwin Island & $10 / 3 / 2000$ & Trans-brown & Intermediate & 628 & $\mathrm{~F}$ & & & & \\
\hline Goodwin Island & $10 / 3 / 2000$ & Trans-brown & Intermediate & 620 & $\mathrm{~F}$ & & & & \\
\hline Goodwin Island & $10 / 3 / 2000$ & Trans-brown & Intermediate & 640 & $\mathrm{~F}$ & & & & \\
\hline Goodwin Island & $10 / 3 / 2000$ & Trans-brown & Intermediate & 600 & $\mathrm{~F}$ & & & & \\
\hline Goodwin Island & $10 / 3 / 2000$ & Transparent & New & 620 & $\mathrm{~F}$ & & & & \\
\hline Goodwin Island & $10 / 3 / 2000$ & Transparent & New & 610 & $\mathrm{~F}$ & & & & \\
\hline Guinea Marsh & $10 / 3 / 2000$ & Brown & Old & 580 & $\mathrm{~F}$ & 617.4666667 & & & \\
\hline Guinea Marsh & $10 / 3 / 2000$ & Brown & Old & 608 & $\mathrm{~F}$ & & & & \\
\hline Guinea Marsh & $10 / 3 / 2000$ & Brown & Old & 610 & $\mathrm{~F}$ & & & & \\
\hline Guinea Marsh & $10 / 3 / 2000$ & Brown & Old & 620 & $\mathrm{~F}$ & & & & \\
\hline Guinea Marsh & $10 / 3 / 2000$ & Brown & Old & 600 & $\mathrm{~F}$ & & & & \\
\hline Guinea Marsh & $10 / 3 / 2000$ & Brown-white & Old & 638 & $\mathrm{~F}$ & & & & \\
\hline Guinea Marsh & $10 / 3 / 2000$ & Trans-brown & Intermediate & 642 & $\mathrm{~F}$ & & & & \\
\hline Guinea Marsh & $10 / 3 / 2000$ & Trans-brown & Intermediate & 620 & $\mathrm{~F}$ & & & & \\
\hline Guinea Marsh & $10 / 3 / 2000$ & Trans-brown & Intermediate & 600 & $\mathrm{~F}$ & & & & \\
\hline Guinea Marsh & $10 / 3 / 2000$ & Trans-brown & Intermediate & 620 & $\mathrm{~F}$ & & & & \\
\hline Guinea Marsh & $10 / 3 / 2000$ & Trans-brown & Intermediate & 620 & $\mathrm{~F}$ & & & & \\
\hline Guinea Marsh & $10 / 3 / 2000$ & Trans-brown & Intermediate & 630 & $\mathrm{~F}$ & & & & \\
\hline Guinea Marsh & $10 / 3 / 2000$ & Trans-brown & Intermediate & 606 & $\mathrm{~F}$ & & & & \\
\hline Guinea Marsh & $10 / 3 / 2000$ & Trans-brown & Intermediate & 620 & $\mathrm{~F}$ & & & & \\
\hline Guinea Marsh & $10 / 3 / 2000$ & Transparent & New & 610 & $\mathrm{~F}$ & & & & \\
\hline Guinea Marsh & $10 / 3 / 2000$ & Transparent & New & 620 & $\mathrm{~F}$ & & & & \\
\hline Guinea Marsh & $10 / 3 / 2000$ & Transparent & New & 640 & $\mathrm{~F}$ & & & & \\
\hline Guinea Marsh & $10 / 3 / 2000$ & Transparent & New & 620 & $\mathrm{~F}$ & & & & \\
\hline Guinea Marsh & $10 / 3 / 2000$ & Transparent & New & 624 & $\mathrm{~F}$ & & & & \\
\hline Guinea Marsh & $10 / 3 / 2000$ & Transparent & New & 606 & $\mathrm{~F}$ & & & & \\
\hline Guinea Marsh & $10 / 3 / 2000$ & Transparent & New & 630 & $\mathrm{~F}$ & & & & \\
\hline Guinea Marsh & $10 / 3 / 2000$ & Transparent & New & 620 & $\mathrm{~F}$ & & & & \\
\hline Guinea Marsh & $10 / 3 / 2000$ & Transparent & New & 620 & $\mathrm{~F}$ & & & & \\
\hline Guinea Marsh & $10 / 3 / 2000$ & Transparent & New & 620 & $\mathrm{~F}$ & & & & \\
\hline Guinea Marsh & $10 / 3 / 2000$ & Transparent & New & 600 & $\mathrm{~F}$ & & & & \\
\hline Guinea Marsh & $10 / 3 / 2000$ & Trans-white & Intermediate/old & 620 & $\mathrm{~F}$ & & & & \\
\hline Guinea Marsh & $10 / 3 / 2000$ & Trans-white & Intermediate/old & 620 & $\mathrm{~F}$ & & & & \\
\hline Guinea Marsh & $10 / 3 / 2000$ & White-opaque & Very old & 620 & $\mathrm{~F}$ & & & & \\
\hline Guinea Marsh & $10 / 3 / 2000$ & White-opaque & Very old & 620 & $\mathrm{~F}$ & & & & \\
\hline Guinea Marsh & $10 / 3 / 2000$ & White-opaque & Very old & 620 & $\mathrm{~F}$ & & & & \\
\hline Goodwin Island & $11 / 14 / 2000$ & Brown & Old & 610 & $\mathrm{~F}$ & & 615.2972973 & & 615.5 \\
\hline Goodwin Island & $11 / 14 / 2000$ & Brown & Old & 606 & $\mathrm{~F}$ & & & & \\
\hline Goodwin Island & $11 / 14 / 2000$ & Brown-white & Old & 604 & $\mathrm{~F}$ & & & & \\
\hline Goodwin Island & $11 / 14 / 2000$ & Transparent & New & 612 & $\mathrm{~F}$ & & & & \\
\hline Goodwin Island & $11 / 14 / 2000$ & Transparent & New & 632 & $\mathrm{~F}$ & & & & \\
\hline Goodwin Island & $11 / 14 / 2000$ & Transparent & New & 620 & $\mathrm{~F}$ & & & & \\
\hline Goodwin Island & $11 / 14 / 2000$ & Transparent & New & 620 & $\mathrm{~F}$ & & & & \\
\hline
\end{tabular}


Appendix A (continued)

\begin{tabular}{|c|c|c|c|c|c|c|c|c|c|}
\hline Site & Date & Color & Age & $\begin{array}{l}\text { Size } \\
(\mu \mathrm{m})\end{array}$ & Sex & $\begin{array}{l}\text { Mean size } \\
\text { Guinea }(\mu \mathrm{m})\end{array}$ & $\begin{array}{l}\text { Mean size } \\
\text { Goodwin }(\mu \mathrm{m})\end{array}$ & $\begin{array}{l}\text { Mean size } \\
\text { Dameron }(\mu \mathrm{m})\end{array}$ & $\begin{array}{l}\text { Grand mean } \\
\text { size }(\mu \mathrm{m})\end{array}$ \\
\hline Goodwin Island & $11 / 14 / 2000$ & Transparent & New & 636 & $\mathrm{~F}$ & & & & \\
\hline Goodwin Island & $11 / 14 / 2000$ & Transparent & New & 620 & F & & & & \\
\hline Goodwin Island & $11 / 14 / 2000$ & Transparent & New & 626 & F & & & & \\
\hline Goodwin Island & $11 / 14 / 2000$ & Transparent & New & 620 & F & & & & \\
\hline Goodwin Island & $11 / 14 / 2000$ & Transparent & New & 604 & F & & & & \\
\hline Goodwin Island & $11 / 14 / 2000$ & Transparent & New & 602 & F & & & & \\
\hline Goodwin Island & $11 / 14 / 2000$ & Transparent & New & 626 & F & & & & \\
\hline Goodwin Island & $11 / 14 / 2000$ & Transparent & New & 630 & F & & & & \\
\hline Goodwin Island & $11 / 14 / 2000$ & Transparent & New & 600 & F & & & & \\
\hline Goodwin Island & $11 / 14 / 2000$ & Trans-brown & Intermediate & 610 & F & & & & \\
\hline Goodwin Island & $11 / 14 / 2000$ & Trans-brown & Intermediate & 620 & F & & & & \\
\hline Goodwin Island & $11 / 14 / 2000$ & Trans-brown & Intermediate & 602 & $\mathrm{~F}$ & & & & \\
\hline Goodwin Island & $11 / 14 / 2000$ & Trans-brown & Intermediate & 600 & F & & & & \\
\hline Goodwin Island & $11 / 14 / 2000$ & Trans-brown & Intermediate & 620 & F & & & & \\
\hline Goodwin Island & $11 / 14 / 2000$ & Trans-brown & Intermediate & 620 & $\mathrm{~F}$ & & & & \\
\hline Goodwin Island & $11 / 14 / 2000$ & Trans-brown & Intermediate & 640 & F & & & & \\
\hline Goodwin Island & $11 / 14 / 2000$ & Trans-brown & Intermediate & 600 & F & & & & \\
\hline Goodwin Island & $11 / 14 / 2000$ & Trans-brown & Intermediate & 616 & F & & & & \\
\hline Goodwin Island & $11 / 14 / 2000$ & Trans-brown & Intermediate & 626 & F & & & & \\
\hline Goodwin Island & $11 / 14 / 2000$ & Trans-brown & Intermediate & 600 & F & & & & \\
\hline Goodwin Island & $11 / 14 / 2000$ & Trans-brown & Intermediate & 600 & F & & & & \\
\hline Goodwin Island & $11 / 14 / 2000$ & Trans-brown & Intermediate & 620 & $\mathrm{~F}$ & & & & \\
\hline Goodwin Island & $11 / 14 / 2000$ & Transparent & New & 618 & $\mathrm{~F}$ & & & & \\
\hline Goodwin Island & $11 / 14 / 2000$ & Transparent & New & 626 & F & & & & \\
\hline Goodwin Island & $11 / 14 / 2000$ & Transparent & New & 632 & F & & & & \\
\hline Goodwin Island & $11 / 14 / 2000$ & Transparent & New & 614 & F & & & & \\
\hline Goodwin Island & $11 / 14 / 2000$ & Transparent & New & 600 & F & & & & \\
\hline Goodwin Island & $11 / 14 / 2000$ & Very brown & Very old & 620 & F & & & & \\
\hline Goodwin Island & $11 / 14 / 2000$ & White-opaque & Very old & 604 & F & & & & \\
\hline Goodwin Island & $11 / 14 / 2000$ & White-opaque & Very old & 610 & F & & & & \\
\hline Guinea Marsh & $11 / 14 / 2000$ & Brown & Old & 620 & F & 616.1818182 & & & \\
\hline Guinea Marsh & $11 / 14 / 2000$ & Brown & Old & 618 & F & & & & \\
\hline Guinea Marsh & $11 / 14 / 2000$ & Brown & Old & 620 & F & & & & \\
\hline Guinea Marsh & $11 / 14 / 2000$ & Brown & Old & 624 & F & & & & \\
\hline Guinea Marsh & $11 / 14 / 2000$ & Brown & Old & 610 & F & & & & \\
\hline Guinea Marsh & $11 / 14 / 2000$ & Brown & Old & 600 & F & & & & \\
\hline Guinea Marsh & $11 / 14 / 2000$ & Brown & Old & 620 & F & & & & \\
\hline Guinea Marsh & $11 / 14 / 2000$ & Trans-brown & Intermediate & 620 & F & & & & \\
\hline Guinea Marsh & $11 / 14 / 2000$ & Trans-brown & Intermediate & 606 & F & & & & \\
\hline Guinea Marsh & $11 / 14 / 2000$ & Transparent & New & 622 & F & & & & \\
\hline Guinea Marsh & $11 / 14 / 2000$ & Very brown & Very old & 618 & $\mathrm{~F}$ & & & & \\
\hline Goodwin Island & $12 / 18 / 2000$ & Brown-white & Old & 624 & F & & 619.6 & & 618.7575758 \\
\hline Goodwin Island & $12 / 18 / 2000$ & Brown-white & Old & 614 & F & & & & \\
\hline Goodwin Island & $12 / 18 / 2000$ & Brown-white & Old & 610 & F & & & & \\
\hline Goodwin Island & $12 / 18 / 2000$ & Trans-brown & Intermediate & 620 & $\mathrm{~F}$ & & & & \\
\hline Goodwin Island & $12 / 18 / 2000$ & Trans-brown & Intermediate & 606 & F & & & & \\
\hline Goodwin Island & $12 / 18 / 2000$ & Transparent & New & 620 & F & & & & \\
\hline Goodwin Island & $12 / 18 / 2000$ & Transparent & New & 630 & F & & & & \\
\hline Goodwin Island & $12 / 18 / 2000$ & Transparent & New & 640 & F & & & & \\
\hline Goodwin Island & $12 / 18 / 2000$ & Transparent & New & 602 & $\mathrm{~F}$ & & & & \\
\hline Goodwin Island & $12 / 18 / 2000$ & Transparent & New & 622 & F & & & & \\
\hline Goodwin Island & $12 / 18 / 2000$ & Transparent & New & 640 & F & & & & \\
\hline Goodwin Island & $12 / 18 / 2000$ & Transparent & New & 626 & $\mathrm{~F}$ & & & & \\
\hline Goodwin Island & $12 / 18 / 2000$ & Transparent & New & 616 & F & & & & \\
\hline
\end{tabular}


Appendix A (continued)

\begin{tabular}{|c|c|c|c|c|c|c|c|c|c|}
\hline Site & Date & Color & Age & $\begin{array}{l}\text { Size } \\
(\mu \mathrm{m})\end{array}$ & Sex & $\begin{array}{l}\text { Mean size } \\
\text { Guinea }(\mu \mathrm{m})\end{array}$ & $\begin{array}{l}\text { Mean size } \\
\text { Goodwin }(\mu \mathrm{m})\end{array}$ & $\begin{array}{l}\text { Mean size } \\
\text { Dameron }(\mu \mathrm{m})\end{array}$ & $\begin{array}{l}\text { Grand mean } \\
\text { size }(\mu \mathrm{m})\end{array}$ \\
\hline Goodwin Island & $12 / 18 / 2000$ & Transparent & New & 620 & $\mathrm{~F}$ & & & & \\
\hline Goodwin Island & $12 / 18 / 2000$ & Transparent & New & 622 & $\mathrm{~F}$ & & & & \\
\hline Goodwin Island & $12 / 18 / 2000$ & Transparent & New & 624 & $\mathrm{~F}$ & & & & \\
\hline Goodwin Island & $12 / 18 / 2000$ & Transparent & New & 610 & $\mathrm{~F}$ & & & & \\
\hline Goodwin Island & $12 / 18 / 2000$ & Transparent & New & 600 & $\mathrm{~F}$ & & & & \\
\hline Goodwin Island & $12 / 18 / 2000$ & Transparent & New & 620 & $\mathrm{~F}$ & & & & \\
\hline Goodwin Island & $12 / 18 / 2000$ & Transparent & New & 626 & $\mathrm{~F}$ & & & & \\
\hline Guinea Marsh & $12 / 18 / 2000$ & Brown & Old & 600 & $\mathrm{~F}$ & 618.3913043 & & & \\
\hline Guinea Marsh & $12 / 18 / 2000$ & Brown & Old & 600 & $\mathrm{~F}$ & & & & \\
\hline Guinea Marsh & $12 / 18 / 2000$ & Brown & Old & 620 & $\mathrm{~F}$ & & & & \\
\hline Guinea Marsh & $12 / 18 / 2000$ & Brown & Old & 612 & $\mathrm{~F}$ & & & & \\
\hline Guinea Marsh & $12 / 18 / 2000$ & Brown & Old & 614 & $\mathrm{~F}$ & & & & \\
\hline Guinea Marsh & $12 / 18 / 2000$ & Brown & Old & 620 & $\mathrm{~F}$ & & & & \\
\hline Guinea Marsh & $12 / 18 / 2000$ & Brown & Old & 620 & $\mathrm{~F}$ & & & & \\
\hline Guinea Marsh & $12 / 18 / 2000$ & Brown & Old & 618 & $\mathrm{~F}$ & & & & \\
\hline Guinea Marsh & $12 / 18 / 2000$ & Brown & Old & 610 & $\mathrm{~F}$ & & & & \\
\hline Guinea Marsh & $12 / 18 / 2000$ & Brown & Old & 624 & $\mathrm{~F}$ & & & & \\
\hline Guinea Marsh & $12 / 18 / 2000$ & Brown & Old & 640 & $\mathrm{~F}$ & & & & \\
\hline Guinea Marsh & $12 / 18 / 2000$ & Brown & Old & 620 & $\mathrm{~F}$ & & & & \\
\hline Guinea Marsh & $12 / 18 / 2000$ & Brown-white & Old & 626 & $\mathrm{~F}$ & & & & \\
\hline Guinea Marsh & $12 / 18 / 2000$ & Brown-white & Old & 622 & $\mathrm{~F}$ & & & & \\
\hline Guinea Marsh & $12 / 18 / 2000$ & Brown-white & Old & 628 & $\mathrm{~F}$ & & & & \\
\hline Guinea Marsh & $12 / 18 / 2000$ & Brown-white & Old & 616 & $\mathrm{~F}$ & & & & \\
\hline Guinea Marsh & $12 / 18 / 2000$ & Trans-brown & Intermediate & 622 & $\mathrm{~F}$ & & & & \\
\hline Guinea Marsh & $12 / 18 / 2000$ & Transparent & New & 640 & $\mathrm{~F}$ & & & & \\
\hline Guinea Marsh & $12 / 18 / 2000$ & Transparent & New & 610 & $\mathrm{~F}$ & & & & \\
\hline Guinea Marsh & $12 / 18 / 2000$ & Transparent & New & 618 & $\mathrm{~F}$ & & & & \\
\hline Guinea Marsh & $12 / 18 / 2000$ & Transparent & New & 618 & $\mathrm{~F}$ & & & & \\
\hline Guinea Marsh & $12 / 18 / 2000$ & Transparent & New & 624 & $\mathrm{~F}$ & & & & \\
\hline Guinea Marsh & $12 / 18 / 2000$ & Transparent & New & 620 & $\mathrm{~F}$ & & & & \\
\hline Guinea Marsh & $12 / 18 / 2000$ & Transparent & New & 614 & $\mathrm{~F}$ & & & & \\
\hline Guinea Marsh & $12 / 18 / 2000$ & Transparent & New & 620 & $\mathrm{~F}$ & & & & \\
\hline Guinea Marsh & $12 / 18 / 2000$ & Transparent & New & 620 & $\mathrm{~F}$ & & & & \\
\hline Guinea Marsh & $12 / 18 / 2000$ & Transparent & New & 624 & $\mathrm{~F}$ & & & & \\
\hline Guinea Marsh & $12 / 18 / 2000$ & Transparent & New & 604 & $\mathrm{~F}$ & & & & \\
\hline Guinea Marsh & $12 / 18 / 2000$ & Transparent & New & 624 & $\mathrm{~F}$ & & & & \\
\hline Guinea Marsh & $12 / 18 / 2000$ & Trans-white & Intermediate/old & 620 & $\mathrm{~F}$ & & & & \\
\hline Guinea Marsh & $12 / 18 / 2000$ & Trans-white & Intermediate/old & 616 & $\mathrm{~F}$ & & & & \\
\hline Guinea Marsh & $12 / 18 / 2000$ & Trans-white & Intermediate/old & 600 & $\mathrm{~F}$ & & & & \\
\hline Guinea Marsh & $12 / 18 / 2000$ & Trans-white & Intermediate/old & 632 & $\mathrm{~F}$ & & & & \\
\hline Guinea Marsh & $12 / 18 / 2000$ & Very brown & Very old & 636 & $\mathrm{~F}$ & & & & \\
\hline Guinea Marsh & $12 / 18 / 2000$ & Very brown & Very old & 626 & $\mathrm{~F}$ & & & & \\
\hline Guinea Marsh & $12 / 18 / 2000$ & Very brown & Very old & 630 & $\mathrm{~F}$ & & & & \\
\hline Guinea Marsh & $12 / 18 / 2000$ & Very brown & Very old & 626 & $\mathrm{~F}$ & & & & \\
\hline Guinea Marsh & $12 / 18 / 2000$ & Very brown & Very old & 600 & $\mathrm{~F}$ & & & & \\
\hline Guinea Marsh & $12 / 18 / 2000$ & White-opaque & Very old & 600 & $\mathrm{~F}$ & & & & \\
\hline Guinea Marsh & $12 / 18 / 2000$ & White-opaque & Very old & 608 & $\mathrm{~F}$ & & & & \\
\hline Guinea Marsh & $12 / 18 / 2000$ & White-opaque & Very old & 624 & $\mathrm{~F}$ & & & & \\
\hline Guinea Marsh & $12 / 18 / 2000$ & White-opaque & Very old & 600 & $\mathrm{~F}$ & & & & \\
\hline Guinea Marsh & $12 / 18 / 2000$ & White-opaque & Very old & 616 & $\mathrm{~F}$ & & & & \\
\hline Guinea Marsh & $12 / 18 / 2000$ & White-opaque & Very old & 600 & $\mathrm{~F}$ & & & & \\
\hline Guinea Marsh & $12 / 18 / 2000$ & White-opaque & Very old & 640 & $\mathrm{~F}$ & & & & \\
\hline Guinea Marsh & $12 / 18 / 2000$ & White-opaque & Very old & 624 & $\mathrm{~F}$ & & & & \\
\hline
\end{tabular}




\section{Appendix B}

Modern Loxoconcha matagordensis shell size data, Florida Bay

\begin{tabular}{|c|c|c|c|c|c|}
\hline Site & Date & Color & Age & $\begin{array}{l}\text { Size } \\
(\mu \mathrm{m})\end{array}$ & Sex \\
\hline FB12\#2.3 & Feb-98 & Dead? & Dead? & 610 & $\mathrm{~F}$ \\
\hline FB12\#2.4 & Feb-98 & Dead? & Dead? & 600 & $\mathrm{~F}$ \\
\hline FB12\#1.2 & Feb-98 & Dead? & Dead? & 650 & $\mathrm{~F}$ \\
\hline FB16\#2.6 & Feb-98 & Dead? & Dead? & 716 & M \\
\hline FB16\#2.7 & Feb-98 & Dead? & Dead? & 700 & M \\
\hline FB1A\#3.1 & Jul-98 & Trans-brown? & Dead? & 616 & F \\
\hline FB5\#1.2 & Feb-98 & Trans-yellow & Intermediate & 622 & $\mathrm{~F}$ \\
\hline FB16\#1.2 & Feb-98 & Trans-yellow & Intermediate & 620 & $\mathrm{~F}$ \\
\hline FB5\#5.4 & Feb-98 & Trans-yellow & Intermediate & 704 & $\mathrm{~F}$ \\
\hline FB21\#1.1 & Feb-98 & Trans-brown & Intermediate & 610 & $\mathrm{~F}$ \\
\hline FB11\#2.2 & Feb-98 & Trans-brown & Intermediate & 594 & $\mathrm{~F}$ \\
\hline FB11\#2.3 & Feb-98 & Trans-brown & Intermediate & 606 & $\mathrm{~F}$ \\
\hline FB26\#1.1 & Feb-98 & Trans-brown & Intermediate & 622 & $\mathrm{~F}$ \\
\hline FB26\#1.7 & Feb-98 & Trans-brown & Intermediate & 586 & $\mathrm{~F}$ \\
\hline FB17\#1.1 & Jul-98 & Trans-brown & Intermediate & 570 & $\mathrm{~F}$ \\
\hline FB6A\#1.2 & Jul-98 & Trans-brown & Intermediate & 592 & $\mathrm{~F}$ \\
\hline FB16\#3.14 & Feb-98 & Trans-brown & Intermediate & 558 & $\mathrm{~F}$ \\
\hline FB16\#3.15 & Feb-98 & Trans-brown & Intermediate & 582 & $\mathrm{~F}$ \\
\hline FB16\#3.16 & Feb-98 & Trans-brown & Intermediate & 594 & $\mathrm{~F}$ \\
\hline FB16\#3.23 & Feb-98 & Trans-brown & Intermediate & 602 & $\mathrm{~F}$ \\
\hline FB16\#3.26 & Feb-98 & Trans-brown & Intermediate & 560 & $\mathrm{~F}$ \\
\hline FB16\#3.32 & Feb-98 & Trans-brown & Intermediate & 560 & $\mathrm{~F}$ \\
\hline FB16\#3.35 & Feb-98 & Trans-brown & Intermediate & 598 & $\mathrm{~F}$ \\
\hline FB16\#3.36 & Feb-98 & Trans-brown & Intermediate & 584 & $\mathrm{~F}$ \\
\hline FB16\#3.38 & Feb-98 & Trans-brown & Intermediate & 562 & $\mathrm{~F}$ \\
\hline FB16\#3.41 & Feb-98 & Trans-brown & Intermediate & 590 & $\mathrm{~F}$ \\
\hline FB16\#3.45 & Feb-98 & Trans-brown & Intermediate & 580 & $\mathrm{~F}$ \\
\hline FB16\#1.4 & Jul-98 & Trans-brown & Intermediate & 552 & $\mathrm{~F}$ \\
\hline FB16\#1.8 & Jul-98 & Trans-brown & Intermediate & 576 & $\mathrm{~F}$ \\
\hline FB16\#1.11 & Jul-98 & Trans-opaque & Intermediate & 590 & F \\
\hline FB16\#1.13 & Jul-98 & Trans-brown & Intermediate & 600 & F \\
\hline FB16\#1.14 & Jul-98 & Trans-brown & Intermediate & 590 & $\mathrm{~F}$ \\
\hline FB16\#1.15 & Jul-98 & Trans-brown & Intermediate & 580 & F \\
\hline FB16\#1.18 & Jul-98 & Trans-brown & Intermediate & 570 & F \\
\hline FB16\#1.22 & Jul-98 & Trans-brown & Intermediate & 582 & $\mathrm{~F}$ \\
\hline FB16\#1.23 & Jul-98 & Trans-brown & Intermediate & 588 & F \\
\hline FB16\#1.32 & Jul-98 & Trans-brown & Intermediate & 650 & F \\
\hline FB16\#1.36 & Jul-98 & Trans-brown & Intermediate & 570 & $\mathrm{~F}$ \\
\hline FB12\#1.1 & Feb-00 & Trans-brown & Intermediate & 690 & F \\
\hline FB12\#1.7 & Feb-00 & Trans-brown & Intermediate & 620 & $\mathrm{~F}$ \\
\hline FB12\#1.9 & Feb-00 & Trans-brown & Intermediate & 604 & $\mathrm{~F}$ \\
\hline FB12\#1.19 & Feb-00 & Trans-brown & Intermediate & 584 & $\mathrm{~F}$ \\
\hline FB12\#2.5 & Feb-98 & Trans-brown & Intermediate & 692 & M \\
\hline FB17\#3.2 & Feb-98 & Trans-yellow & Intermediate & 690 & M \\
\hline FB21\#1.4 & Feb-98 & Trans-brown & Intermediate & 718 & M \\
\hline FB20\#1.2 & Feb-98 & Trans-brown & Intermediate & 686 & M \\
\hline FB8A\#2.1 & Jul-98 & Trans-brown & Intermediate & 650 & M \\
\hline FB26\#2.1 & Jul-98 & Trans-brown & Intermediate & 676 & M \\
\hline FB16\#3.1 & Feb-98 & Trans-brown & Intermediate & 670 & M \\
\hline
\end{tabular}

Appendix B (continued)

\begin{tabular}{|c|c|c|c|c|c|}
\hline Site & Date & Color & Age & $\begin{array}{l}\text { Size } \\
(\mu \mathrm{m}) \\
\end{array}$ & Sex \\
\hline FB16\#3.2 & Feb-98 & Trans-brown & Intermediate & 682 & M \\
\hline FB16\#3.11 & Feb-98 & Trans-brown & Intermediate & 720 & M \\
\hline FB16\#3.12 & Feb-98 & Trans-brown & Intermediate & 700 & M \\
\hline FB16\#3.30 & Feb-98 & Trans-brown & Intermediate & 684 & M \\
\hline FB16\#3.43 & Feb-98 & Trans-brown & Intermediate & 680 & M \\
\hline FB16\#3.46 & Feb-98 & Trans-brown & Intermediate & 670 & M \\
\hline FB16\#3.57 & Feb-98 & Trans-brown & Intermediate & 714 & M \\
\hline FB16\#1.27 & Jul-98 & Trans-brown & Intermediate & 676 & M \\
\hline FB16\#1.33 & Jul-98 & Trans-brown & Intermediate & 630 & M \\
\hline FB16\#1.45 & Jul-98 & Trans-brown & Intermediate & 664 & M \\
\hline FB1A\#3.2 & Jul-98 & Trans-brown? & Intermediate & 600 & \\
\hline FB1\#1.1 & Feb-98 & Trans-yellow & Inter-old & 610 & $\mathrm{~F}$ \\
\hline FB12\#2.2 & Feb-98 & Yellow-brown & Intermediate? & 616 & $\mathrm{~F}$ \\
\hline FB4\#4.1 & Feb-98 & Trans-yellow & Intermediate & 628 & $\mathrm{~F}$ \\
\hline FB17\#2.1 & Feb-98 & Trans & New & 608 & $\mathrm{~F}$ \\
\hline FB5\#1.1 & Feb-98 & Trans & New & 624 & $\mathrm{~F}$ \\
\hline FB16\#1.1 & Feb-98 & Trans & New & 626 & $\mathrm{~F}$ \\
\hline FB5\#5.2 & Feb-98 & Trans & New & 626 & $\mathrm{~F}$ \\
\hline FB12\#2.1 & Feb-98 & Trans & New & 620 & $\mathrm{~F}$ \\
\hline FB16\#2.1 & Feb-98 & Trans & New & 620 & $\mathrm{~F}$ \\
\hline FB16\#2.5 & Feb-98 & Trans & New & 626 & $\mathrm{~F}$ \\
\hline FB20\#5.2 & Feb-98 & Trans & New & 594 & $\mathrm{~F}$ \\
\hline FB26\#1.2 & Feb-98 & Trans & New & 600 & $\mathrm{~F}$ \\
\hline FB26\#1.3 & Feb-98 & Trans & New & 626 & $\mathrm{~F}$ \\
\hline FB26\#1.4 & Feb-98 & Trans & New & 572 & $\mathrm{~F}$ \\
\hline FB26\#1.6 & Feb-98 & Trans & New & 614 & $\mathrm{~F}$ \\
\hline FB17\#1.2 & Jul-98 & Trans & New & 556 & $\mathrm{~F}$ \\
\hline FB17\#1.3 & Jul-98 & Trans & New & 568 & $\mathrm{~F}$ \\
\hline FB17\#1.4 & Jul-98 & Trans & New & 592 & $\mathrm{~F}$ \\
\hline FB17\#1.5 & Jul-98 & Trans & New & 574 & $\mathrm{~F}$ \\
\hline FB17\#1.6 & Jul-98 & Trans & New & 584 & $\mathrm{~F}$ \\
\hline FB6A\#1.3 & Jul-98 & Trans & New & 590 & $\mathrm{~F}$ \\
\hline FB6A\#1.4 & Jul-98 & Trans & New & 600 & $\mathrm{~F}$ \\
\hline FB12\#1.1 & Oct-99 & White-trans & New & 604 & $\mathrm{~F}$ \\
\hline FB16\#3.3 & Feb-98 & Trans & New & 604 & $\mathrm{~F}$ \\
\hline FB16\#3.5 & Feb-98 & Trans & New & 600 & $\mathrm{~F}$ \\
\hline FB16\#3.9 & Feb-98 & Trans & New & 580 & $\mathrm{~F}$ \\
\hline FB16\#3.18 & Feb-98 & Trans & New & 566 & $\mathrm{~F}$ \\
\hline FB16\#3.19 & Feb-98 & Trans & New & 584 & $\mathrm{~F}$ \\
\hline FB16\#3.20 & Feb-98 & Trans & New & 604 & $\mathrm{~F}$ \\
\hline FB16\#3.21 & Feb-98 & Trans & New & 620 & $\mathrm{~F}$ \\
\hline FB16\#3.22 & Feb-98 & Trans-opaque & New & 624 & $\mathrm{~F}$ \\
\hline FB16\#3.24 & Feb-98 & Trans & New & 596 & $\mathrm{~F}$ \\
\hline FB16\#3.31 & Feb-98 & Trans & New & 588 & $\mathrm{~F}$ \\
\hline FB16\#3.37 & Feb-98 & Trans & New & 586 & $\mathrm{~F}$ \\
\hline FB16\#3.42 & Feb-98 & Trans & New & 600 & $\mathrm{~F}$ \\
\hline FB16\#3.47 & Feb-98 & Trans & New & 584 & $\mathrm{~F}$ \\
\hline FB16\#3.50 & Feb-98 & Trans & New & 624 & $\mathrm{~F}$ \\
\hline FB16\#3.51 & Feb-98 & Trans & New & 598 & $\mathrm{~F}$ \\
\hline FB16\#3.52 & Feb-98 & Trans & New & 632 & $\mathrm{~F}$ \\
\hline FB16\#3.53 & Feb-98 & Trans & New & 606 & $\mathrm{~F}$ \\
\hline FB16\#1.1 & Jul-98 & Trans & New & 560 & $\mathrm{~F}$ \\
\hline FB16\#1.2 & Jul-98 & Trans & New & 568 & $\mathrm{~F}$ \\
\hline
\end{tabular}

(continued on next page) 


\begin{tabular}{|c|c|c|c|c|c|}
\hline Site & Date & Color & Age & $\begin{array}{l}\text { Size } \\
(\mu \mathrm{m})\end{array}$ & Sex \\
\hline FB16\#1.3 & Jul-98 & Trans & New & 610 & $\mathrm{~F}$ \\
\hline FB16\#1.6 & Jul-98 & Trans & New & 624 & $\mathrm{~F}$ \\
\hline FB16\#1.9 & Jul-98 & Trans & New & 600 & $\mathrm{~F}$ \\
\hline FB16\#1.16 & Jul-98 & Trans-white & New & 590 & $\mathrm{~F}$ \\
\hline FB16\#1.17 & Jul-98 & Trans & New & 562 & $\mathrm{~F}$ \\
\hline FB16\#1.19 & Jul-98 & Trans & New & 582 & $\mathrm{~F}$ \\
\hline FB16\#1.25 & Jul-98 & Trans & New & 542 & $\mathrm{~F}$ \\
\hline FB16\#1.28 & Jul-98 & Trans & New & 586 & $\mathrm{~F}$ \\
\hline FB16\#1.29 & Jul-98 & Trans & New & 598 & $\mathrm{~F}$ \\
\hline FB16\#1.35 & Jul-98 & Trans & New & 610 & $\mathrm{~F}$ \\
\hline FB16\#1.37 & Jul-98 & Trans & New & 582 & $\mathrm{~F}$ \\
\hline FB16\#1.38 & Jul-98 & Trans & New & 600 & $\mathrm{~F}$ \\
\hline FB16\#1.39 & Jul-98 & Trans & New & 580 & $\mathrm{~F}$ \\
\hline FB16\#1.40 & Jul-98 & Trans & New & 622 & $\mathrm{~F}$ \\
\hline FB16\#1.41 & Jul-98 & Trans & New & 564 & $\mathrm{~F}$ \\
\hline FB16\#1.42 & Jul-98 & Trans & New & 640 & $\mathrm{~F}$ \\
\hline FB16\#1.44 & Jul-98 & Trans & New & 550 & $\mathrm{~F}$ \\
\hline FB12\#1.2 & Feb-00 & Trans & New & 548 & $\mathrm{~F}$ \\
\hline FB12\#1.4 & Feb-00 & Trans & New & 540 & $\mathrm{~F}$ \\
\hline FB12\#1.12 & Feb-00 & Trans & New & 604 & $\mathrm{~F}$ \\
\hline FB12\#1.13 & Feb-00 & Trans & New & 594 & $\mathrm{~F}$ \\
\hline FB12\#1.15 & Feb-00 & Trans & New & 580 & $\mathrm{~F}$ \\
\hline FB12\#1.16 & Feb-00 & Trans & New & 586 & $\mathrm{~F}$ \\
\hline FB12\#1.18 & Feb-00 & Trans & New & 580 & $\mathrm{~F}$ \\
\hline FB26\#1.9 & Feb-98 & Trans & New & 678 & $\mathrm{M}$ \\
\hline FB17\#1.7 & Jul-98 & Trans & New & 672 & $\mathrm{M}$ \\
\hline FB17\#1.8 & Jul-98 & Trans & New & 654 & M \\
\hline FB17\#1.9 & Jul-98 & Trans & New & 648 & M \\
\hline FB6A\#1.6 & Jul-98 & Trans & New & 702 & $\mathrm{M}$ \\
\hline FB18\#2.2 & Jul-98 & Trans & New & 680 & M \\
\hline FB18\#2.3 & Jul-98 & Trans & New & 708 & M \\
\hline FB16\#3.6 & Feb-98 & Trans & New & 684 & $\mathrm{M}$ \\
\hline FB16\#3.8 & Feb-98 & Trans & New & 662 & $\mathrm{M}$ \\
\hline FB16\#3.17 & Feb-98 & Trans & New & 684 & $\mathrm{M}$ \\
\hline FB16\#3.28 & Feb-98 & Trans & New & 646 & M \\
\hline FB16\#3.33 & Feb-98 & Trans & New & 700 & $\mathrm{M}$ \\
\hline FB16\#3.39 & Feb-98 & Trans & New & 682 & $\mathrm{M}$ \\
\hline FB16\#3.54 & Feb-98 & Trans & New & 696 & M \\
\hline FB16\#3.55 & Feb-98 & Trans & New & 702 & $\mathrm{M}$ \\
\hline FB16\#3.56 & Feb-98 & Trans & New & 688 & $\mathrm{M}$ \\
\hline FB16\#1.5 & Jul-98 & Trans & New & 680 & $\mathrm{M}$ \\
\hline FB16\#1.10 & Jul-98 & Trans & New & 696 & $\mathrm{M}$ \\
\hline FB16\#1.20 & Jul-98 & Trans-white & New & 670 & $\mathrm{M}$ \\
\hline FB16\#1.24 & Jul-98 & Trans & New & 702 & $\mathrm{M}$ \\
\hline FB16\#1.26 & Jul-98 & Trans & New & 680 & $\mathrm{M}$ \\
\hline FB16\#1.30 & Jul-98 & Trans & New & 658 & $\mathrm{M}$ \\
\hline FB16\#1.31 & Jul-98 & Trans & New & 680 & $\mathrm{M}$ \\
\hline FB16\#1.34 & Jul-98 & Trans & New & 640 & $\mathrm{M}$ \\
\hline FB16\#1.43 & Jul-98 & Trans-opaque & New & 696 & M \\
\hline FB12\#1.2 & Feb-99 & Trans & New & 660 & M \\
\hline FB5\#5.3 & Feb-98 & Trans-yellow & New-inter & 614 & $\mathrm{~F}$ \\
\hline FB12\#1.3 & Oct-99 & Trans-brown & New-inter & 598 & $\mathrm{~F}$ \\
\hline FB21\#1.3 & Feb-98 & Trans-brown & New-inter & 724 & $\mathrm{M}$ \\
\hline
\end{tabular}

Appendix B (continued)

\begin{tabular}{|c|c|c|c|c|c|}
\hline Site & Date & Color & Age & $\begin{array}{l}\text { Size } \\
(\mu \mathrm{m})\end{array}$ & Sex \\
\hline FB26\#1.10 & Feb-98 & Trans-brown & New-inter & 700 & M \\
\hline FB26\#1.11 & Feb-98 & Trans-brown & New-inter & 702 & M \\
\hline FB1\#5.1 & Feb-98 & Yellow-brown & Old & 608 & $\mathrm{~F}$ \\
\hline FB5\#5.1 & Feb-98 & Yellow-brown & Old & 600 & $\mathrm{~F}$ \\
\hline FB11\#5.2 & Feb-98 & Yellow-brown & Old & 606 & $\mathrm{~F}$ \\
\hline FB11\#5.3 & Feb-98 & Yellow-brown & Old & 586 & $\mathrm{~F}$ \\
\hline FB20\#3.1 & Feb-98 & Brown & Old & 596 & $\mathrm{~F}$ \\
\hline FB20\#3.2 & Feb-98 & Brown & Old & 584 & $\mathrm{~F}$ \\
\hline FB26\#2.1 & Feb-98 & Yellow-opaque & Old & 620 & $\mathrm{~F}$ \\
\hline FB16\#2.3 & Feb-98 & Yellow-brown & Old & 606 & $\mathrm{~F}$ \\
\hline FB21\#1.2 & Feb-98 & Brown & Old & 620 & $\mathrm{~F}$ \\
\hline FB20\#1.1 & Feb-98 & Brown & Old & 594 & $\mathrm{~F}$ \\
\hline FB11\#2.1 & Feb-98 & Brown & Old & 602 & $\mathrm{~F}$ \\
\hline FB26\#1.5 & Feb-98 & Brown & Old & 602 & $\mathrm{~F}$ \\
\hline FB26\#1.8 & Feb-98 & Brown & Old & 596 & $\mathrm{~F}$ \\
\hline FB20A\#3.1 & Jul-98 & Brown? & Old & 574 & $\mathrm{~F}$ \\
\hline FB12\#1.2 & Oct-99 & Brown & Old & 558 & $\mathrm{~F}$ \\
\hline FB16\#3.4 & Feb-98 & Opaque-white & Old & 580 & $\mathrm{~F}$ \\
\hline FB16\#3.7 & Feb-98 & White-opaque & Old & 580 & $\mathrm{~F}$ \\
\hline FB16\#3.10 & Feb-98 & White-opaque & Old & 622 & $\mathrm{~F}$ \\
\hline FB16\#3.25 & Feb-98 & Brown & Old & 604 & $\mathrm{~F}$ \\
\hline FB16\#3.34 & Feb-98 & Brown & Old & 574 & $\mathrm{~F}$ \\
\hline FB16\#3.40 & Feb-98 & Brown & Old & 574 & $\mathrm{~F}$ \\
\hline FB16\#3.44 & Feb-98 & Brown & Old & 580 & $\mathrm{~F}$ \\
\hline FB16\#3.48 & Feb-98 & Brown & Old & 600 & $\mathrm{~F}$ \\
\hline FB16\#3.49 & Feb-98 & Brown & Old & 622 & $\mathrm{~F}$ \\
\hline FB16\#1.7 & Jul-98 & White-opaque & Old & 570 & $\mathrm{~F}$ \\
\hline FB16\#1.12 & Jul-98 & Brown & Old & 580 & $\mathrm{~F}$ \\
\hline FB16\#1.21 & Jul-98 & Brown & Old & 580 & $\mathrm{~F}$ \\
\hline FB12\#1.2 & Feb-99 & Brown & Old & 604 & $\mathrm{~F}$ \\
\hline FB12\#1.3 & Feb-00 & White-opaque & Old & 620 & $\mathrm{~F}$ \\
\hline FB12\#1.5 & Feb-00 & Brown & Old & 596 & $\mathrm{~F}$ \\
\hline FB12\#1.6 & Feb-00 & Brown & Old & 600 & $\mathrm{~F}$ \\
\hline FB12\#1.8 & Feb-00 & Brown & Old & 604 & $\mathrm{~F}$ \\
\hline FB12\#1.10 & Feb-00 & White & Old & 580 & $\mathrm{~F}$ \\
\hline FB12\#1.11 & Feb-00 & Brown & Old & 600 & $\mathrm{~F}$ \\
\hline FB12\#1.14 & Feb-00 & Brown & Old & 596 & $\mathrm{~F}$ \\
\hline FB12\#1.17 & Feb-00 & Brown & Old & 590 & $\mathrm{~F}$ \\
\hline FB20\#5.3 & Feb-98 & Brown & Old & 672 & $\mathrm{M}$ \\
\hline FB6A\#1.5 & Jul-98 & Brown? & Old & 684 & M \\
\hline FB16\#3.13 & Feb-98 & Brown & Old & 650 & M \\
\hline FB16\#3.27 & Feb-98 & Brown & Old & 684 & M \\
\hline FB16\#3.29 & Feb-98 & White-opaque & Old & 690 & $\mathrm{M}$ \\
\hline FB12\#1.1 & Feb-99 & Brown & Old & 680 & $\mathrm{M}$ \\
\hline FB11\#5.1 & Feb-98 & Yellow-brown & Old? & 596 & $\mathrm{~F}$ \\
\hline FB20\#5.1 & Feb-98 & Brown & Old? & 616 & $\mathrm{~F}$ \\
\hline FB18\#2.1 & Jul-98 & Brown? & Old? & 604 & $\mathrm{~F}$ \\
\hline FB12\#1.1 & Feb-98 & Brown-opaque & Very old & 598 & $\mathrm{~F}$ \\
\hline FB6A\#1.1 & Jul-98 & Brown-opaque & Very old & 580 & $\mathrm{~F}$ \\
\hline FB12\#1.3 & Feb-98 & Brown-opaque & Very old & 678 & M \\
\hline FB17\#3.1 & Feb-98 & White-opaque & Very old, dead? & 580 & $\mathrm{~F}$ \\
\hline
\end{tabular}




\section{Appendix C}

Living Loxoconcha matagordensis shell chemistry data

\begin{tabular}{|c|c|c|c|c|c|c|c|c|c|c|c|c|c|c|c|}
\hline Location & Date & $\begin{array}{l}\text { Salinity } \\
\text { (ppt) }\end{array}$ & $\begin{array}{l}\text { Measured } \\
\text { temperature } \\
\left({ }^{\circ} \mathrm{C}\right)\end{array}$ & $\begin{array}{l}\text { Secretion } \\
\text { temperature } \\
\left({ }^{\circ} \mathrm{C}\right)\end{array}$ & vpi & Species & Molt & $\begin{array}{l}\text { Valve/ } \\
\text { Carapace }\end{array}$ & Sex & $\begin{array}{l}\mathrm{Mg} / \mathrm{Ca} \\
(\mathrm{mmol} / \mathrm{mol})\end{array}$ & $\begin{array}{l}\mathrm{Sr} / \mathrm{Ca} \\
(\mathrm{mmol} / \mathrm{mol})\end{array}$ & $\begin{array}{l}\mathrm{Na} / \mathrm{Ca} \\
(\mathrm{mmol} / \mathrm{mol})\end{array}$ & $\begin{array}{l}\text { Weight } \\
(\mathrm{mg})\end{array}$ & $\begin{array}{l}\text { Mean } \\
\mathrm{Mg} / \mathrm{Ca} \\
\text { adult }\end{array}$ & $\begin{array}{l}\text { Mean } \\
\mathrm{Mg} / \mathrm{Ca} \mathrm{A} \\
\text { and A-1 }\end{array}$ \\
\hline MMS STA \#58 & 6/19/1996 & & 20 & 20 & 5 & spp. & A & $\mathrm{R}$ & $\mathrm{M}$ & 20.1718323 & 3.40655 & 6.146833 & 5.70794 & & \\
\hline MMS STA \#96 & 6/19/1996 & & 20 & 20 & 5 & williamsi & A & $\mathrm{L}$ & $\mathrm{M}$ & 29.2511582 & 3.64734 & 22.76568 & 6.222238 & & \\
\hline MMS STA \#R11 & 6/19/1996 & & 20 & 20 & 2 & spp. & A & $\mathrm{L}$ & $\mathrm{F}$ & 21.3152137 & 3.29635 & 11.74143 & 13.80238 & & \\
\hline MMS STA \#R12 & 6/19/1996 & & 20 & 20 & 4 & spp. & A & $\mathrm{L}$ & $\mathrm{M}$ & 21.3712786 & 3.12904 & 9.055816 & 10.5859 & & \\
\hline MMS STA \#R7 & 6/19/1996 & & 20 & 20 & 3 & spp. & A & $\mathrm{R}$ & $\mathrm{M}$ & 24.3586577 & 3.28888 & 13.20077 & 10.93334 & & \\
\hline Goodwin Island & 2/9/1999 & 21 & 6 & 13.45 & & matagordensis & A & 10 cars & & 25.27 & 4.18 & 17.45 & & 25.09902781 & 25.09903 \\
\hline Goodwin Island & 2/9/1999 & 21 & 6 & 13.45 & & matagordensis & A & 10 cars & & 28.66 & 3.98 & 17.95 & & & \\
\hline Goodwin Island & 2/9/1999 & 21 & 6 & 13.45 & & matagordensis & A & 10 cars & & 27.81 & 3.96 & 14.19 & & & \\
\hline Goodwin Island & 2/9/1999 & 21 & 6 & 13.45 & & matagordensis & A & 10 cars & & 18.77 & 3.95 & 13.21 & & & \\
\hline Goodwin Island & 2/9/1999 & 21 & 6 & 13.45 & & matagordensis & A & 10 cars & & 21.46 & 3.64 & 14.45 & & & \\
\hline Goodwin Island & 2/9/1999 & 21 & 6 & 13.45 & & matagordensis & A & 10 cars & & 29.13 & 4.12 & 15.64 & & & \\
\hline Goodwin Island & 2/9/1999 & 21 & 6 & 13.45 & & matagordensis & A & 10 cars & & 22.18 & 3.80 & 23.08 & & & \\
\hline Guinea marsh & 2/9/1999 & 21 & 6 & 13.45 & & matagordensis & A & 10 cars & & 32.38 & 3.91 & 14.39 & & 25.69571962 & 25.69572 \\
\hline Guinea marsh & 2/9/1999 & 21 & 6 & 13.45 & & matagordensis & A & 10 cars & & 25.16 & 4.03 & 14.99 & & & \\
\hline Guinea marsh & 2/9/1999 & 21 & 6 & 13.45 & & matagordensis & A & 10 cars & & 20.82 & 3.67 & 13.27 & & & \\
\hline Guinea marsh & 2/9/1999 & 21 & 6 & 13.45 & & matagordensis & A & 10 cars & & 27.24 & 3.94 & 16.38 & & & \\
\hline Guinea marsh & 2/9/1999 & 21 & 6 & 13.45 & & matagordensis & A & 10 cars & & 26.84 & 3.96 & 14.69 & & & \\
\hline Dameron Marsh & 3/1/1999 & 16.1 & 12 & 12 & & matagordensis & A & 2 cars & & 23.26 & 4.33 & 15.08 & & 23.40532506 & 23.40533 \\
\hline Dameron Marsh & 3/1/1999 & 16.1 & 12 & 12 & & matagordensis & A & 2 cars & & 23.55 & 4.01 & 14.73 & & & \\
\hline Guinea Marsh & 4/27/1999 & 19 & 15 & 15 & & matagordensis & A & 10 cars & & 23.54 & 3.67 & 15.89 & & 24.29949503 & 24.29950 \\
\hline Guinea Marsh & 4/27/1999 & 19 & 15 & 15 & & matagordensis & A & 10 cars & & 23.04 & 3.93 & 14.23 & & & \\
\hline Guinea Marsh & 4/27/1999 & 19 & 15 & 15 & & matagordensis & A & 10 cars & & 21.94 & 3.89 & 14.32 & & & \\
\hline Guinea Marsh & 4/27/1999 & 19 & 15 & 15 & & matagordensis & A & 10 cars & & 29.30 & 3.90 & 15.48 & & & \\
\hline Guinea Marsh & 4/27/1999 & 19 & 15 & 15 & & matagordensis & A & 10 cars & & 20.18 & 3.53 & 14.04 & & & \\
\hline Guinea Marsh & 4/27/1999 & 19 & 15 & 15 & & matagordensis & A & 10 cars & & 23.48 & 3.91 & 13.23 & & & \\
\hline Guinea Marsh & 4/27/1999 & 19 & 15 & 15 & & matagordensis & A & 10 cars & & 21.92 & 3.93 & 16.50 & & & \\
\hline Guinea Marsh & 4/27/1999 & 19 & 15 & 15 & & matagordensis & A & 10 cars & & 25.01 & 3.81 & 13.20 & & & \\
\hline Guinea Marsh & 4/27/1999 & 19 & 15 & 15 & & matagordensis & A & 10 cars & & 24.96 & 3.58 & 15.39 & & & \\
\hline Guinea Marsh & 4/27/1999 & 19 & 15 & 15 & & matagordensis & A & 10 cars & & 28.07 & 4.10 & 14.95 & & & \\
\hline Guinea Marsh & 4/27/1999 & 19 & 15 & 15 & & matagordensis & A & 10 cars & & 25.86 & 3.87 & 14.15 & & & \\
\hline Guinea Marsh & 4/27/1999 & 19 & 15 & 15 & & matagordensis & A & 10 cars & & 27.90 & 3.37 & 15.06 & & & \\
\hline Guinea Marsh & 4/27/1999 & 19 & 15 & 15 & & matagordensis & A & 10 cars & & 20.27 & 3.56 & 13.16 & & & \\
\hline Guinea Marsh & 4/27/1999 & 19 & 15 & 15 & & matagordensis & A & 10 cars & & 24.71 & 3.77 & 14.88 & & & \\
\hline Guinea Marsh & $5 / 12 / 1999$ & 17.1 & 20 & 20 & 2 & matagordensis & A & A & $\mathrm{M}$ & 36.53 & 4.03 & 22.57 & & 38.72167149 & 39.40969 \\
\hline Guinea Marsh & $5 / 12 / 1999$ & 17.1 & 20 & 20 & 2 & matagordensis & A & & M & 41.25 & 4.37 & 19.07 & & & \\
\hline Guinea Marsh & $5 / 12 / 1999$ & 17.1 & 20 & 20 & 2 & matagordensis & A & & $\mathrm{F}$ & 38.38 & 4.47 & 15.74 & & & \\
\hline
\end{tabular}




\begin{tabular}{|c|c|c|c|c|c|c|c|c|c|c|c|c|c|c|c|}
\hline Location & Date & $\begin{array}{l}\text { Salinity } \\
\text { (ppt) }\end{array}$ & $\begin{array}{l}\text { Measured } \\
\text { temperature } \\
\left({ }^{\circ} \mathrm{C}\right)\end{array}$ & $\begin{array}{l}\text { Secretion } \\
\text { temperature } \\
\left({ }^{\circ} \mathrm{C}\right)\end{array}$ & vpi & Species & Molt & $\begin{array}{l}\text { Valve/ } \\
\text { Carapace }\end{array}$ & Sex & $\begin{array}{l}\mathrm{Mg} / \mathrm{Ca} \\
(\mathrm{mmol} / \mathrm{mol})\end{array}$ & $\begin{array}{l}\mathrm{Sr} / \mathrm{Ca} \\
(\mathrm{mmol} / \mathrm{mol})\end{array}$ & $\begin{array}{l}\mathrm{Na} / \mathrm{Ca} \\
(\mathrm{mmol} / \mathrm{mol})\end{array}$ & $\begin{array}{l}\text { Weight } \\
(\mathrm{mg})\end{array}$ & $\begin{array}{l}\mathrm{Mean} \\
\mathrm{Mg} / \mathrm{Ca} \\
\text { adult }\end{array}$ & $\begin{array}{l}\text { Mean } \\
\mathrm{Mg} / \mathrm{Ca} \mathrm{A} \\
\text { and } \mathrm{A}-1\end{array}$ \\
\hline Guinea Marsh & $5 / 12 / 1999$ & 17.1 & 20 & 20 & & matagordensis & A-1 & & M & 41.47 & 4.29 & 18.76 & & & \\
\hline Goodwin Island & 6/3/1999 & 20 & 20 & 20 & 2 & matagordensis & A & & $\mathrm{F}$ & 32.67 & 3.85 & 19.00 & & 33.64 & 33.64 \\
\hline Goodwin Island & 6/3/1999 & 20 & 20 & 20 & 2 & matagordensis & A & & $\mathrm{F}$ & 32.09 & 4.20 & 17.91 & & & \\
\hline Guinea Marsh & 6/3/1999 & 17.8 & 20 & 20 & 2 & matagordensis & A & & $\mathrm{F}$ & 36.15 & 4.15 & 19.34 & & & \\
\hline \#8.1 & 6/7/1999 & 30.50 & 16.90 & 16.90 & 2 & williamsi & A & Right & $\mathrm{M}$ & 17.7708091 & 2.9251 & 11.5515 & & 29.54033492 & 28.43837 \\
\hline$\# 8.1$ & 6/7/1999 & 30.50 & 16.90 & 16.90 & 2 & williamsi & A-1 & Right & $\mathrm{M}$ & 28.4446475 & 3.63493 & 21.52269 & & & \\
\hline CB5.4 & 6/7/1999 & 21.70 & 18.50 & 18.50 & 1 & williamsi & A & Left & $\mathrm{M}$ & 22.6010436 & 3.38446 & 11.53694 & & & \\
\hline CB5.4 & 6/7/1999 & 18.50 & 21.70 & 21.70 & 2 & williamsi & A-1 & Left & $\mathrm{M}$ & 17.8458195 & 3.07734 & 18.14812 & & & \\
\hline CB6.1 & 6/7/1999 & 22.30 & 20.50 & 20.50 & 2 & williamsi & A & Right & $\mathrm{F}$ & 33.5595833 & 3.58502 & 15.1389 & & & \\
\hline CB6.1 & 6/7/1999 & 22.30 & 20.50 & 20.50 & 1 & williamsi & A & Left & $\mathrm{M}$ & 28.2502705 & 3.56791 & 12.94282 & & & \\
\hline CB6.1 & 6/7/1999 & 22.30 & 20.50 & 20.50 & 1 & williamsi & A & Left & $\mathrm{M}$ & 35.3846242 & 3.44399 & 13.252 & & & \\
\hline CB6.1 & 6/7/1999 & 22.30 & 20.50 & 20.50 & 2 & williamsi & A-1 & Right & $\mathrm{M}$ & 25.2827488 & 3.39934 & 13.89081 & & & \\
\hline CB6.1 & 6/7/1999 & 22.30 & 20.50 & 20.50 & 2 & williamsi & A-1 & Right & M & 31.0586932 & 3.34798 & 53.43278 & & & \\
\hline CB6.4 & 6/7/1999 & 27.40 & 19.20 & 19.20 & 1 & williamsi & A & Carapace & $\mathrm{M}$ & 21.2117721 & 3.28178 & 11.98603 & & & \\
\hline CB6.4 & 6/7/1999 & 27.40 & 19.20 & 19.20 & 2 & williamsi & A & Right & $\mathrm{M}$ & 29.0218435 & 3.65074 & 13.33604 & & & \\
\hline CB6.4 & 6/7/1999 & 27.40 & 19.20 & 19.20 & 2 & williamsi & A & Left & $\mathrm{M}$ & 27.7328107 & 3.71128 & 13.69128 & & & \\
\hline CB6.4 & 6/7/1999 & 27.40 & 19.20 & 19.20 & 1 & williamsi & A-1 & Right & $\mathrm{M}$ & 30.2924317 & 3.64279 & 42.5504 & & & \\
\hline CB6.4 & 6/7/1999 & 27.40 & 19.20 & 19.20 & 2 & williamsi & A-1 & Left & $\mathrm{F}$ & 29.4219679 & 3.37545 & 28.35716 & & & \\
\hline CB7.3E & 6/7/1999 & 28.90 & 19.80 & 19.80 & 2 & williamsi & A & Right & $\mathrm{M}$ & 24.0476891 & 3.43832 & 14.83851 & & & \\
\hline CB7.3E & 6/7/1999 & 28.90 & 19.80 & 19.80 & 2 & williamsi & A & Carapace & $\mathrm{M}$ & 38.8038738 & 3.89791 & 21.10997 & & & \\
\hline CB7.3E & 6/7/1999 & 28.90 & 19.80 & 19.80 & 2 & williamsi & A & Carapace & $\mathrm{F}$ & 27.277179 & 3.48078 & 13.26126 & & & \\
\hline CB7.3E & 6/7/1999 & 28.90 & 19.80 & 19.80 & 2 & williamsi & A & Carapace & $\mathrm{M}$ & 35.7927438 & 3.50073 & 15.23238 & & & \\
\hline CB7.3E & 6/7/1999 & 28.90 & 19.80 & 19.80 & 1 & williamsi & A & Carapace & M & 26.6317106 & 3.46148 & 17.84451 & & & \\
\hline CB7.3E & 6/7/1999 & 28.90 & 19.80 & 19.80 & 3 & williamsi & A & Carapace & $\mathrm{M}$ & 22.2926318 & 3.51278 & 18.2388 & & & \\
\hline CB7.3E & 6/7/1999 & 28.90 & 19.80 & 19.80 & 1 & williamsi & A & Left & $\mathrm{M}$ & 23.6305519 & 3.11057 & 11.19043 & & & \\
\hline CB7.3E & 6/7/1999 & 28.90 & 19.80 & 19.80 & 2 & williamsi & A-1 & Right & M & 22.606778 & 3.33508 & 13.42305 & & & \\
\hline CB5.4 & 6/8/1999 & 21.70 & 18.50 & 18.50 & 1 & williamsi & A & $\mathrm{L}$ & $\mathrm{F}$ & 44.7777401 & 4.1473 & 14.60473 & 7.262151 & & \\
\hline CB6.1 & 6/8/1999 & 22.30 & 20.50 & 20.50 & 2 & williamsi & A & $\mathrm{L}$ & $\mathrm{M}$ & 37.4013438 & 3.26558 & 7.8422 & 8.327827 & & \\
\hline CB6.4 & 6/8/1999 & 27.40 & 19.20 & 19.20 & 2 & williamsi & A & $\mathrm{R}$ & M & 24.5859998 & 3.37396 & 13.42411 & 9.135408 & & \\
\hline CB7.3E & 6/8/1999 & 28.90 & 19.80 & 19.80 & 1 & williamsi & A & $\mathrm{R}$ & $\mathrm{M}$ & 40.1215594 & 3.52403 & 13.32882 & 12.29965 & & \\
\hline CB7.3E & 6/8/1999 & 28.90 & 19.80 & 19.80 & 2 & williamsi & A & $\mathrm{L}$ & $\mathrm{M}$ & 24.7882579 & 3.51274 & 9.294947 & 9.372412 & & \\
\hline CB7.3E & 6/8/1999 & 28.90 & 19.80 & 19.80 & 1 & williamsi & A & $\mathrm{R}$ & $\mathrm{F}$ & 37.5840474 & 3.45336 & 9.426554 & 8.691313 & & \\
\hline CB7.3E & 6/8/1999 & 28.90 & 19.80 & 19.80 & 2 & williamsi & A & $\mathrm{L}$ & $\mathrm{M}$ & 26.3614515 & 3.44384 & 9.735881 & 8.355782 & & \\
\hline LE4.3B & 6/8/1999 & 25.30 & 19.80 & 19.80 & 2 & williamsi & A & Right & $\mathrm{M}$ & 29.7981661 & 3.09302 & 14.16399 & & & \\
\hline LE4.3 & 6/9/1999 & 18.80 & 20.30 & 20.30 & 2 & williamsi & A-1 & Right & $\mathrm{M}$ & 24.8776814 & 3.80843 & 16.96566 & & & \\
\hline LE4.3 & 6/9/1999 & 18.80 & 20.30 & 20.30 & 2 & williamsi & A-1 & Right & $\mathrm{M}$ & 20.7693916 & 2.95754 & 18.71912 & & & \\
\hline Goodwin Island & 6/22/1999 & 20.2 & 22 & 22 & 1 & matagordensis & A & & $\mathrm{F}$ & 34.09 & 4.33 & 21.12 & & 35.51 & 35.45803 \\
\hline Goodwin Island & 6/22/1999 & 20.2 & 22 & 22 & 2 & matagordensis & A & & $\mathrm{F}$ & 36.93 & 4.15 & 17.77 & & & \\
\hline Goodwin Island & 6/22/1999 & 20.2 & 22 & 22 & & matagordensis & A-1 & & $\mathrm{F}$ & 35.35 & 4.30 & 32.46 & & & \\
\hline Guinea Marsh & 6/22/1999 & 19.5 & 22 & 22 & 1 & matagordensis & A & & $\mathrm{M}$ & 37.87 & 4.14 & 18.78 & & 37.87 & 34.88962 \\
\hline Guinea Marsh & 6/22/1999 & 19.5 & 22 & 22 & 1 & matagordensis & A-1 & & $\mathrm{M}$ & 31.91 & 4.19 & 19.34 & & & \\
\hline Guinea Marsh & 7/6/1999 & 21.5 & 29.2 & 29.2 & 1 & matagordensis & A & & $\mathrm{F}$ & 47.50 & 4.07 & 17.99 & & 49.1269926 & 50.23979 \\
\hline Guinea Marsh & 7/6/1999 & 21.5 & 29.2 & 29.2 & 1 & matagordensis & A & & $\mathrm{F}$ & 50.76 & 3.85 & 19.43 & & & \\
\hline Guinea Marsh & 7/6/1999 & 21.5 & 29.2 & 29.2 & 1 & matagordensis & A-1 & & $\mathrm{M}$ & 52.47 & 4.37 & 20.24 & & & \\
\hline Goodwin Island & 7/20/1999 & 21.5 & 27 & 27 & & matagordensis & A & & $\mathrm{F}$ & 38.6176744 & 4.58497 & 20.1059 & 8.309723 & 46.15248453 & 45.29008244 \\
\hline Guinea Marsh & 7/20/1999 & 19.9 & 27 & 27 & 2 & matagordensis & A & & $\mathrm{F}$ & 39.82 & 4.20 & 17.17 & & & \\
\hline
\end{tabular}




\begin{tabular}{|c|c|c|c|c|c|c|c|}
\hline Guinea Marsh & 7/20/1999 & 19.9 & 27 & 27 & 2 & matagordensis & A \\
\hline Guinea Marsh & 7/20/1999 & 19.9 & 27 & 27 & 2 & matagordensis & A \\
\hline Guinea Marsh & $7 / 20 / 1999$ & 19.9 & 27 & 27 & 2 & matagordensis & A \\
\hline Guinea Marsh & 7/20/1999 & 19.9 & 27 & 27 & 3 & matagordensis & A \\
\hline Guinea Marsh & 7/20/1999 & 19.9 & 27 & 27 & 3 & matagordensis & A \\
\hline Guinea Marsh & $7 / 20 / 1999$ & 19.9 & 27 & 27 & 2 & matagordensis & A \\
\hline Guinea Marsh & $7 / 20 / 1999$ & 19.9 & 27 & 27 & 2 & matagordensis & A \\
\hline Guinea Marsh & 7/20/1999 & 19.9 & 27 & 27 & 1 & matagordensis & A-1 \\
\hline Guinea Marsh & 7/20/1999 & 19.9 & 27 & 27 & 1 & matagordensis & A-1 \\
\hline Goodwin Island & $8 / 3 / 1999$ & 21 & 26 & 26 & & matagordensis & A \\
\hline Guinea Marsh & $8 / 3 / 1999$ & 19.9 & 25.5 & 25.5 & 1 & matagordensis & A \\
\hline Guinea Marsh & $8 / 3 / 1999$ & 19.9 & 25.5 & 25.5 & 3 & matagordensis & A \\
\hline Guinea Marsh & $8 / 3 / 1999$ & 19.9 & 25.5 & 25.5 & 4 & matagordensis & A \\
\hline Guinea Marsh & $8 / 3 / 1999$ & 19.9 & 25.5 & 25.5 & 3 & matagordensis & A \\
\hline Guinea Marsh & 8/3/1999 & 19.9 & 25.5 & 25.5 & 3 & matagordensis & A \\
\hline Guinea Marsh & $8 / 3 / 1999$ & 19.9 & 25.5 & 25.5 & 4 & matagordensis & A \\
\hline Guinea Marsh & $8 / 3 / 1999$ & 19.9 & 25.5 & 25.5 & 4 & matagordensis & A \\
\hline Guinea Marsh & $8 / 3 / 1999$ & 19.9 & 25.5 & 25.5 & 2 & matagordensis & A-1 \\
\hline Guinea Marsh & $8 / 3 / 1999$ & 19.9 & 25.5 & 25.5 & 2 & matagordensis & A- 1 \\
\hline Bogue Sound & 8/13/1999 & 35.5 & 30 & 30 & 2 & matagordensis & A \\
\hline Bogue Sound & 8/13/1999 & 35.5 & 30 & 30 & 2 & matagordensis & A \\
\hline Bogue Sound & 8/13/1999 & 35.5 & 30 & 30 & 2 & matagordensis & A \\
\hline Bogue Sound & 8/13/1999 & 35.5 & 30 & 30 & 2 & matagordensis & A \\
\hline Bogue Sound & 8/13/1999 & 35.5 & 30 & 30 & 2 & matagordensis & A \\
\hline Bogue Sound & 8/13/1999 & 35.5 & 30 & 30 & 2 & matagordensis & A \\
\hline Bogue Sound & 8/13/1999 & 35.5 & 30 & 30 & 2 & matagordensis & A \\
\hline Bogue Sound & 8/13/1999 & 35.5 & 30 & 30 & 2 & matagordensis & A \\
\hline Guinea Marsh & $8 / 17 / 1999$ & 21.3 & 26.8 & 26.8 & & matagordensis & A \\
\hline Guinea Marsh & $8 / 17 / 1999$ & 21.3 & 26.8 & 26.8 & & matagordensis & A \\
\hline 4 CB. 11 & 9/9/1999 & 23.50 & 23.00 & 23.00 & 4 & williamsi & A \\
\hline 4CB. 14 & 9/9/1999 & 23.50 & 23.00 & 23.00 & 3 & williamsi & A \\
\hline 4CB. 16 & 9/9/1999 & 23.50 & 23.00 & 23.00 & 4 & williamsi & A \\
\hline 4CB. 3 & 9/9/1999 & 23.50 & 23.00 & 23.00 & 4 & williamsi & A \\
\hline 4CB. 5 & 9/9/1999 & 23.50 & 23.00 & 23.00 & 3 & williamsi & A \\
\hline 4CB.7 & 9/9/1999 & 23.50 & 23.00 & 23.00 & 3 & williamsi & A \\
\hline 4CB. 8 & 9/9/1999 & 23.50 & 23.00 & 23.00 & 3 & williamsi & A \\
\hline 4CB. 9 & 9/9/1999 & 23.50 & 23.00 & 23.00 & 5 & williamsi & A \\
\hline 5CB. 1 & 9/9/1999 & 23.50 & 22.70 & 22.70 & 4 & williamsi & A \\
\hline 5 CВ. 2 & 9/9/1999 & 23.50 & 22.70 & 22.70 & 4 & williamsi & A \\
\hline 5 CB. 3 & 9/9/1999 & 23.50 & 22.70 & 22.70 & 4 & williamsi & A \\
\hline 5 CB. 4 & 9/9/1999 & 23.50 & 22.70 & 22.70 & 3 & williamsi & A \\
\hline 5CB. 5 & 9/9/1999 & 23.50 & 22.70 & 22.70 & 4 & williamsi & A \\
\hline 5CB. 6 & 9/9/1999 & 23.50 & 22.70 & 22.70 & 6 & williamsi & A \\
\hline 3 CB. 10 & 9/11/1999 & 23.50 & 23.30 & 23.30 & 5 & williamsi & A \\
\hline 3CB.11 & 9/11/1999 & 23.50 & 23.30 & 23.30 & 5 & williamsi & A \\
\hline 3СВ. 2 & 9/11/1999 & 23.50 & 23.30 & 23.30 & 5 & williamsi & A \\
\hline 3CB. 5 & 9/11/1999 & 23.50 & 23.30 & 23.30 & 4 & williamsi & A \\
\hline 3CB.7 & 9/11/1999 & 23.50 & 23.30 & 23.30 & 5 & williamsi & A \\
\hline 3СВ.9 & 9/11/1999 & 23.50 & 23.30 & 23.30 & 6 & williamsi & A \\
\hline
\end{tabular}

$\begin{array}{ll}39.82 & 4.20 \\ 48.59 & 4.46 \\ 47.42 & 4.18 \\ 44.51 & 4.46 \\ 58.10 & 4.36 \\ 49.64 & 4.53 \\ 48.85 & 4.35 \\ 43.31 & 4.02 \\ 39.51 & 4.23 \\ 52.1527656 & 3.20599 \\ 46.00 & 4.23 \\ 44.76 & 4.62 \\ 44.38 & 4.72 \\ 45.72 & 4.55 \\ 40.93 & 4.50 \\ 46.81 & 4.61 \\ 50.04 & 4.33 \\ 47.39 & 4.33 \\ 50.97 & 4.49 \\ 44.10 & 4.14 \\ 40.26 & 4.23 \\ 44.01 & 4.36 \\ 45.00 & 4.21 \\ 38.80 & 4.23 \\ 43.18 & 4.14 \\ 46.23 & 4.17 \\ 47.44 & 4.20 \\ 44.1559449 & 3.84951 \\ 40.0038517 & 4.51393 \\ 34.6553671 & 3.71993 \\ 34.9781433 & 3.84298 \\ 37.7363332 & 3.77752 \\ 48.9380419 & 3.60716 \\ 39.7655671 & 4.30991 \\ 45.3878827 & 4.26755 \\ 35.9724146 & 4.15899 \\ 39.3895908 & 3.49205 \\ 23.2748309 & 3.2373 \\ 41.0394686 & 3.2722 \\ 50.2747731 & 3.95052 \\ 24.00934 & 3.38102 \\ 38.201106 & 3.65057 \\ 33.7616681 & 3.38279 \\ 39.4008719 & 3.63225 \\ 29.0908099 & 3.43318 \\ 28.3169509 & 3.5296 \\ 31.434376 & 3.85596 \\ 31.5284326 & 3.85102 \\ 36.0898909 & 4.17594 \\ & \end{array}$

$\begin{array}{ll}17.17 & \\ 19.12 & 7.3 \\ 20.01 & 7.5 \\ 18.21 & 7.3 \\ 19.01 & 8.0 \\ 20.28 & 9.3 \\ 20.20 & 7.9\end{array}$

19.34

24.45

$\begin{array}{llll}12.65017 & 7.640268 & 46.34829943 & 46.91412\end{array}$

18.38

18.47

17.43

17.79

17.68

19.79

22.60

40.67
18.98

20.38

33.84

20.23

21.36

21.65

$\begin{array}{llll}16.17257 & 4.674272 & 42.07989826 & 42.07990\end{array}$

$16.3357 \quad 8.176594$

$\begin{array}{llll}11.09409 & 7.9 & 36.16229299 & 36.16229\end{array}$

$\begin{array}{ll}12.63651 & 4.2\end{array}$

$12.54796 \quad 7.4$

$\begin{array}{ll}12.61866 & 4.3 \\ 10.25821 & 4.1\end{array}$

$\begin{array}{ll}10.25821 & 4.1 \\ 15.83328 & 4.8\end{array}$

$\begin{array}{ll}10.26151 & 6.4\end{array}$

$11.60376 \quad 7.8$

$\begin{array}{ll}10.40084 & 9.2 \\ 11.05275 & 8.6\end{array}$

$\begin{array}{ll}13.73262 & 7.4\end{array}$

$\begin{array}{ll}10.77795 & 8.9\end{array}$

$\begin{array}{ll}11.02844 & 7.4\end{array}$

$10.38423 \quad 6.9$

$12.50319 \quad 8.5$

$13.70511 \quad 10.2$

$10.97745 \quad 9.5$

$13.50269 \quad 7.6$

$12.78602 \quad 6.3$ 


\begin{tabular}{|c|c|c|c|c|c|c|c|c|c|c|c|c|c|c|c|}
\hline Location & Date & $\begin{array}{l}\text { Salinity } \\
\text { (ppt) }\end{array}$ & $\begin{array}{l}\text { Measured } \\
\text { temperature } \\
\left({ }^{\circ} \mathrm{C}\right)\end{array}$ & $\begin{array}{l}\text { Secretion } \\
\text { temperature } \\
\left({ }^{\circ} \mathrm{C}\right)\end{array}$ & vpi & Species & Molt & $\begin{array}{l}\text { Valve/ } \\
\text { Carapace }\end{array}$ & Sex & $\begin{array}{l}\mathrm{Mg} / \mathrm{Ca} \\
(\mathrm{mmol} / \mathrm{mol})\end{array}$ & $\begin{array}{l}\mathrm{Sr} / \mathrm{Ca} \\
(\mathrm{mmol} / \mathrm{mol})\end{array}$ & $\begin{array}{l}\mathrm{Na} / \mathrm{Ca} \\
(\mathrm{mmol} / \mathrm{mol})\end{array}$ & $\begin{array}{l}\text { Weight } \\
(\mathrm{mg})\end{array}$ & $\begin{array}{l}\text { Mean } \\
\mathrm{Mg} / \mathrm{Ca} \\
\text { adult }\end{array}$ & $\begin{array}{l}\text { Mean } \\
\mathrm{Mg} / \mathrm{Ca} \mathrm{A} \\
\text { and A-1 }\end{array}$ \\
\hline Guinea Marsh & 9/14/1999 & 23 & 24.5 & 24.5 & 3 & matagordensis & A & & $\mathrm{F}$ & 43.59 & 3.91 & 16.76 & 7.7 & 44.68023281 & 44.68023 \\
\hline Guinea Marsh & 9/14/1999 & 23 & 24.5 & 24.5 & 3 & matagordensis & A & & $\mathrm{M}$ & 45.28 & 4.41 & 18.17 & 8.3 & & \\
\hline Guinea Marsh & 9/14/1999 & 23 & 24.5 & 24.5 & 3 & matagordensis & A & & $\mathrm{F}$ & 53.55 & 4.82 & 19.67 & 8.5 & & \\
\hline Guinea Marsh & 9/14/1999 & 23 & 24.5 & 24.5 & 3 & matagordensis & A & & $\mathrm{F}$ & 40.03 & 3.85 & 16.16 & 7.3 & & \\
\hline Guinea Marsh & 9/14/1999 & 23 & 24.5 & 24.5 & 5 & matagordensis & A & & $\mathrm{F}$ & 40.94 & 4.58 & 17.30 & 7.0 & & \\
\hline Guinea Marsh & $10 / 15 / 1999$ & 21 & 16 & 16 & 3 & matagordensis & A & & $\mathrm{F}$ & 31.86 & 4.30 & 16.22 & 8.9 & 34.43084277 & 34.43084 \\
\hline Guinea Marsh & 10/15/1999 & 21 & 16 & 16 & 4 & matagordensis & A & & F & 34.07 & 4.38 & 18.25 & 8.0 & & \\
\hline Guinea Marsh & $10 / 15 / 1999$ & 21 & 16 & 16 & 3 & matagordensis & A & & $\mathrm{F}$ & 28.71 & 3.77 & 15.34 & 9.4 & & \\
\hline Guinea Marsh & 10/15/1999 & 21 & 16 & 16 & 2 & matagordensis & A & & $\mathrm{F}$ & 35.02 & 4.11 & 15.35 & 8.5 & & \\
\hline Guinea Marsh & $10 / 15 / 1999$ & 21 & 16 & 16 & 5 & matagordensis & A & & $\mathrm{F}$ & 42.28 & 4.36 & 16.65 & 7.3 & & \\
\hline Guinea Marsh & $10 / 15 / 1999$ & 21 & 16 & 16 & 3 & matagordensis & A & & $\mathrm{F}$ & 34.64 & 4.09 & 16.74 & 8.8 & & \\
\hline Guinea Marsh & 11/9/1999 & 20 & 13 & 13 & 3 & matagordensis & A & & $\mathrm{M}$ & 32.24 & 4.07 & 16.31 & 8.0 & 28.92525688 & 28.92526 \\
\hline Guinea Marsh & 11/9/1999 & 20 & 13 & 13 & 3 & matagordensis & A & & $\mathrm{F}$ & 26.37 & 3.88 & 13.18 & 7.4 & & \\
\hline Guinea Marsh & 11/9/1999 & 20 & 13 & 13 & 5 & matagordensis & A & & $\mathrm{F}$ & 30.45 & 4.00 & 16.48 & 7.5 & & \\
\hline Guinea Marsh & 11/9/1999 & 20 & 13 & 13 & 3 & matagordensis & A & & $\mathrm{F}$ & 24.94 & 3.99 & 14.35 & 6.6 & & \\
\hline Guinea Marsh & 11/9/1999 & 20 & 13 & 13 & 2 & matagordensis & A & & $\mathrm{M}$ & 30.80 & 4.15 & 14.92 & 8.1 & & \\
\hline Guinea Marsh & 11/9/1999 & 20 & 13 & 13 & 2 & matagordensis & A & & $\mathrm{M}$ & 28.75 & 4.00 & 14.74 & 8.8 & & \\
\hline Guinea Marsh & $12 / 21 / 1999$ & 26 & 9.5 & 9.5 & 5 & matagordensis & A & & $\mathrm{F}$ & 30.75 & 4.17 & 15.32 & 7.7 & 24.95413071 & 24.95413 \\
\hline Guinea Marsh & $12 / 21 / 1999$ & 26 & 9.5 & 9.5 & 4 & matagordensis & A & & $\mathrm{F}$ & 24.66 & 3.97 & 13.59 & 7.5 & & \\
\hline Guinea Marsh & $12 / 21 / 1999$ & 26 & 9.5 & 9.5 & 6 & matagordensis & A & & $\mathrm{F}$ & 24.09 & 4.20 & 14.37 & 7.6 & & \\
\hline Guinea Marsh & $12 / 21 / 1999$ & 26 & 9.5 & 9.5 & 6 & matagordensis & A & & $\mathrm{F}$ & 23.91 & 3.94 & 14.95 & 6.7 & & \\
\hline Guinea Marsh & $12 / 21 / 1999$ & 26 & 9.5 & 9.5 & 6 & matagordensis & A & & $\mathrm{F}$ & 21.35 & 4.14 & 15.62 & 7.5 & & \\
\hline 1007 & $1960 \mathrm{~s}$ & 35 & 10 & 10 & 2 & impressa & A & $1 \mathrm{car}$ & Females & 27.6170204 & 3.86779 & 15.81031 & 7.114429 & 26.58032464 & 26.58032 \\
\hline 1009 & $1960 \mathrm{~s}$ & 35 & 10 & 10 & 5 & impressa & A & $1 \mathrm{car}$ & Mostly & 25.5436289 & 4.12663 & 14.50365 & 6.948336 & & \\
\hline 1175 & $1960 \mathrm{~s}$ & 35 & 7 & 7 & 4 & impressa & A & 2 valves & & 17.6024543 & 2.94165 & 8.811903 & 6.955119 & 17.84959791 & 17.84960 \\
\hline 1175 & $1960 \mathrm{~s}$ & 35 & 7 & 7 & 4 & impressa & A & 2 valves & & 18.0967415 & 3.03503 & 9.432979 & 7.255514 & & \\
\hline 1319 & $1960 \mathrm{~s}$ & 35 & 12 & 12 & 5 & williamsi & A & 2 valves & & 24.6331626 & 3.14567 & 9.869401 & 5.735748 & 24.15084848 & 24.15085 \\
\hline 1319 & $1960 \mathrm{~s}$ & 35 & 12 & 12 & 4 & williamsi & A & 2 valves & & 23.6685344 & 3.08767 & 12.03087 & 7.749565 & & \\
\hline 1340 & $1960 \mathrm{~s}$ & 35 & 10 & 10 & 3 & impressa & A & 2 cars & & 21.9375217 & 3.46483 & 11.92977 & 11.93322 & 23.82517422 & 23.82517 \\
\hline 1340 & $1960 \mathrm{~s}$ & 35 & 10 & 10 & 2 & impressa & A & 2 cars & & 25.7128267 & 3.491 & 16.381 & 11.82737 & & \\
\hline 1415 & $1960 \mathrm{~s}$ & 35 & 13 & 13 & 4 & impressa & A & 1 valve, $1 \mathrm{car}$ & & 26.3513643 & 3.47591 & 13.17942 & 9.759425 & 22.05901627 & 22.05902 \\
\hline 1415 & $1960 \mathrm{~s}$ & 35 & 13 & 13 & 4 & impressa & A & 1 valve, $1 \mathrm{car}$ & & 17.7666682 & 3.55086 & 11.08875 & 8.837932 & & \\
\hline 1423 & $1960 \mathrm{~s}$ & 35 & 16 & 16 & 4 & impressa & A & 1 valve, $1 \mathrm{car}$ & & 21.6526938 & 3.42433 & 10.72918 & 12.77937 & 22.2337484 & 22.23375 \\
\hline 1423 & $1960 \mathrm{~s}$ & 35 & 16 & 16 & 4 & impressa & $\mathrm{A}$ & 1 valve, $1 \mathrm{car}$ & & 22.814803 & 3.36557 & 12.36179 & 9.727965 & & \\
\hline 1425 & $1960 \mathrm{~s}$ & 35 & 15 & 15 & 2 & impressa & A & 4 cars & & 19.8775645 & 3.22813 & 12.78063 & 8.530206 & 21.49945637 & 21.49946 \\
\hline 1425 & $1960 \mathrm{~s}$ & 35 & 15 & 15 & 3 & impressa & A & 4 cars & & 23.0975653 & 2.88928 & 10.49227 & 10.1643 & & \\
\hline 1425 & $1960 \mathrm{~s}$ & 35 & 15 & 15 & 3 & impressa & A & 4 cars & & 19.9331263 & 3.20278 & 11.37684 & 11.84626 & & \\
\hline 1425 & $1960 \mathrm{~s}$ & 35 & 15 & 15 & 4 & impressa & A & 4 cars & & 23.0895694 & 3.26447 & 11.96681 & 10.07488 & & \\
\hline 1431 & $1960 \mathrm{~s}$ & 35 & 16 & 16 & 3 & impressa & A & 1 valve, $1 \mathrm{car}$ & & 26.611434 & 3.33788 & 14.96463 & 9.145316 & 30.73339467 & 30.73339 \\
\hline 1431 & $1960 \mathrm{~s}$ & 35 & 16 & 16 & 3 & impressa & A & 1 valve, $1 \mathrm{car}$ & & 34.8553553 & 3.06652 & 12.9789 & 8.70478 & & \\
\hline 1436 & $1960 \mathrm{~s}$ & 35 & 20 & 20 & 2 & williamsi & A & 3 valves & & 29.5207248 & 3.37029 & 11.9639 & 10.15926 & 31.57929032 & 31.57929 \\
\hline 1436 & $1960 \mathrm{~s}$ & 35 & 20 & 20 & 2 & williamsi & A & 3 valves & & 36.3866462 & 3.37809 & 15.14778 & 9.743105 & & \\
\hline 1436 & $1960 \mathrm{~s}$ & 35 & 20 & 20 & 2 & williamsi & A & 3 valves & & 28.8305 & 3.34325 & 12.24692 & 10.10592 & & \\
\hline 2050 & $1960 \mathrm{~s}$ & 35 & 20 & 20 & 3 & williamsi & A & $1 \mathrm{car}$ & & 36.0706553 & 3.60467 & 12.76168 & 7.446499 & 36.07065534 & 36.07066 \\
\hline 1558 & $1960 \mathrm{~s}$ & 35 & 30 & 30 & 3 & matagordensis & A & 2 valves & & 49.7725972 & 3.90318 & 16.5134 & 6.666085 & 48.38845319 & 48.38845 \\
\hline 1558 & $1960 \mathrm{~s}$ & 35 & 30 & 30 & 3 & matagordensis & A & 2 valves & & 47.0043092 & 4.05498 & 18.54688 & 3.344911 & & \\
\hline
\end{tabular}




\section{References}

Cadot Jr., H.M., Kaesler, R.L., 1977. Magnesium content of calcite in carapaces of benthic marine ostracodes. Paleontol. Contrib. (Univ. Kansas) 87, 1-23.

Cadot Jr., H.M., Van Schmus, W.R., Kaesler, R.L., 1972. Magnesium in calcite of marine Ostracoda. Geol. Soc. Am. Bull. 83, $3519-3522$.

Chivas, A.R., De Deckker, P., Shelley, J.M.G., 1983. Magnesium, strontium, and barium partitioning in nonmarine ostracod shells and their use in paleoenvironmental reconstruction - a preliminary study. In: Maddocks, R.F. (Ed.), Applications of Ostracoda. Univ. Houston Geoscience, pp. 238-249.

Chivas, A.R., De Deckker, P., Shelley, J.M.G., 1986. Magnesium and strontium in non-marine ostracod shells as indicators of paleosalinity and paleotemperature. Palaeogeogr. Palaeoclimatol. Palaeoecol. 54, 43-51.

Corrège, T., 1993. Preliminary results of paleotemperature reconstruction using the magnesium to calcium ratio of deepsea ostracod shells from the Late Quaternary of Site 822 Leg 133 (western Coral Sea). Proc. ODP Sci. Results 133, $175-180$.

Corrège, T., De Deckker, P., 1997. Faunal and geochemical evidence for change in intermediate water temperature and salinity in the western Coral Sea during the late Quaternary. Palaeogeogr. Palaeoclimatol. Palaeoecol. 131, 183-205.

Cronin, T.M., Dowsett, H.J., 1990. A quantitative micropaleontologic method for shallow marine paleoclimatology: application to Pliocene deposits of the Western North Atlantic Ocean. Mar. Micropaleontol. 16, 117-148.

Cronin, T.M., Holmes, C., Wingard, L., Ishman, S.E., Dowsett, H.J., Waibel, N., 2001. Historical trends in epiphytal ostracodes from Florida Bay: Implications for seagrass and macrobenthic algal variability. In: Wardlaw, B. (Ed.), Bull. Am. Paleontol., vol. 361, pp. 163-204.

Cronin, T.M., Dwyer, G.S., Kamiya, T., Schwede, S., Willard, D.A., 2003. Medieval Warm Period, Little Ice Age and 20th century temperature variability from Chesapeake Bay. Glob. Planet. Change 36 (1-2), 17-29.

De Deckker, P., Chivas, A.R., Shelley, J.M.G., 1999. Uptake of Mg and $\mathrm{Sr}$ in the euryhaline ostracod Cyprideis determined from in vitro experiments. Palaeogeogr. Palaeoclimatol. Palaeoecol. $148,105-116$.

Dwyer, G.S., Cronin, T.M., 2001. Ostracode shell chemistry as a paleosalinity proxy in Florida Bay. In: Wardlaw, B.R. (Ed.), Bull. Am. Paleontol., vol. 361, pp. 249-276.

Dwyer, G.S., Cronin, T.M., Baker, P.A., Raymo, M.E., Buzas, J.S., Correge, T., 1995. North Atlantic deepwater temperature change during late Pliocene and late Quaternary climatic cycles. Science 270, 1347-1351.

Dwyer, G.S., Cronin, T.M., Baker, P.A., 2002. Trace elements in marine ostracodes. In: Chivas, A.R., Holmes, J. (Eds.), The
Ostracoda - Applications in Quaternary Research, Am. Geophys. Monogr., vol. 131, pp. 205-225.

Elliott, H.A., Ellison, R., Nichols, M.M., 1966. Distribution of recent Ostracoda in the Rappahannock Estuary, Virginia. Chesap. Sci. 7, 203-207.

Engstrom, D.R., Nelson, S.R., 1991. Paleosalinity from trace metals in fossil ostracodes compared with observational records at Devils Lake, North Dakota, USA. Palaeogeogr. Palaeoclimatol. Palaeoecol. 83, 295-312.

Grossman, S., 1965. Morphology and ecology of two podocopid ostracodes from Redfish Bay, Texas. Micropaleontology 11, $141-150$

Hazel, J.E., 1970. Ostracode zoogeography in the southern Nova Scotian and northern Virginian faunal provinces. U. S. Geol. Surv. Prof. Pap. 529-E (21 pp.).

Holmes, J.A., Chivas, A.R., 2002. Ostracod shell chemistry overview. In: Chivas, A.R., Holmes, J. (Eds.), The Ostracoda - Applications in Quaternary Research, Am. Geophys. Monogr., vol. 131, pp. 185-204.

Kamiya, T., 1988. Contrasting population ecology of two species of Loxoconcha (Ostracoda, Crustacea) in recent Zostera (eelgrass) beds: adaptive differences between phytal and bottom-dwelling species. Micropaleontology 34, 316-331.

Kempf, E., 1986. Index and bibliography of marine Ostracoda: Parts 1 and 2. Sonderveroeffentlichungen. Geol. Inst. Univ. Koeln. (http://ostracoda-on.tripod.com/index.html\#kdo pubs).

King, C.E., Kornicker, L.S., 1970. Ostracoda in Texas bays and lagoons: an ecologic study. Smithson. Contrib. Zool. 24 (92 pp.).

Kondo, H., Toyofuku, T., Ikeya, N., 2005. Mg/Ca ratio in the shells of cultured and natural populations of marine ostracode Xestoleberis hanaii (Crustacea). Palaeogeog., Palaeoclimatol., Palaeoecol. 225, 3-13.

Majoran, S., Agrenius, S., Dwyer, G.S., 1999. The effect of temperature on the geochemical composition of the valves of the ostracod species Krithe praetexta praetexta. Geosound 35, $93-113$

Morales, G.A., 1966. Ecology, distribution, and taxonomy of recent Ostracoda of the Laguna de Terminos, Campeche, Mexico. Universidad Nacional Autonoma de Mexico. Bol.-Inst. Geol. 81 (103 pp.).

Swain, F.M., 1955. Ostracoda from San Antonio Bay. J. Paleontol. 29, 561-646.

Tressler, W.L., Smith, E.M., 1948. An ecological study of seasonal distribution of Ostracoda, Solomons Island, Maryland, Region. Chesap. Biol. Lab. Pub. 71, 1-61.

Valentine, P.C., 1971. Climatic implication of a late Pleistocene ostracode assemblage from southeastern Virginia. U. S. Geol. Surv. Prof. Pap. 683-D (28 pp.).

Wansard, G., De Deckker, P., Julia, R., 1998. Variability in ostracod partition coefficients $\mathrm{D}(\mathrm{Sr})$ and $\mathrm{D}(\mathrm{Mg})$. Implications for lacustrine palaeoenvironmental reconstructions. Chem. Geol. 146, $39-54$. 